\title{
DifFusion AND FLOW on Microscopic Length Scales \\ STUDIED WITH \\ Fluorescence Correlation Spectroscopy
}

\author{
D I S S E R T A T I O N
}

\author{
for the award of the degree \\ "Doctor rerum naturalium" (Dr. rer. nat.) \\ Division of Mathematics and Natural Sciences \\ of the Georg-August-Universität Göttingen
}

submitted by

Christoph Michael Pieper

born in Göttingen, Germany

Göttingen 2012 
Members of the Thesis Committee:

\section{Prof. Dr. J. Enderlein (Reviewer)}

Group of "Single molecule spectroscopy and imaging for Biophysics and Complex systems", 3rd Institute of Physics - Biophysics, Faculty of Physics, Georg-August-University Göttingen

\section{Prof. Dr. H. Grubmüller (Reviewer)}

Department of Theoretical and Computer-based Biophysics, Max Planck Institute for Biophysical Chemistry

\section{Prof. Dr. T. Salditt}

Institute for X-Ray Physics, Faculty of Physics, Georg-August-University Göttingen

Date of the oral examination: 23.10 .2012 


\section{Affidavit}

Hereby, I declare that the presented thesis has been written independently and with no other sources and aids than quoted.

Göttingen, 13.09.2012 



\section{Table of Contents}

$\begin{array}{ll}\text { Abstract } & 7\end{array}$

1 Introduction $\mathbf{9}$

1.1 Introduction to Fluorescence Correlation Spectroscopy . . . . . . . . . . . . . 11

1.2 Molecular Crowding . . . . . . . . . . . . . . . . . . . 12

1.3 Microfluidics . . . . . . . . . . . . . . . . . . . . 14

1.4 Nematic Liquid Crystals . . . . . . . . . . . . . . . . . 16

2 Theoretical Background 19

2.1 Theory of Diffusion . . . . . . . . . . . . . . . . . . . 19

2.1.1 Translational Diffusion . . . . . . . . . . . . . . . . . 19

2.1.2 Rotational Diffusion . . . . . . . . . . . . . . 22

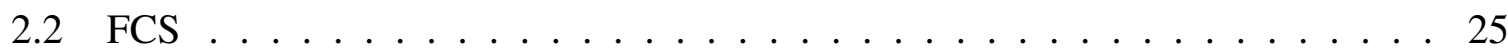

2.2 .1 Fluorescence ......................... 25

2.2.2 Fluorescence Correlation Spectroscopy . . . . . . . . . . . . . . 27

2.2.3 The Correlation Function . . . . . . . . . . . . . . . . . . . 30

2.2 .4 Dye Photophysics . . . . . . . . . . . . . . . . . 31

2.2.5 Rotational Diffusion and Nanosecond timescale FCS . . . . . . . . . 31

2.2 .6 Dual Focus FCS . . . . . . . . . . . . . . . . . . 35

2.2 .7 Measuring Flow Velocities with FCS $\ldots \ldots . \ldots . \ldots 38$

3 Materials and Methods $\quad 39$

3.1 Sample Preparation . . . . . . . . . . . . . . . . . . . . . . . 39

3.1 .1 Labeling of Proteins . . . . . . . . . . . . . . . . 39

3.2 FCS Setup . . . . . . . . . . . . . . . . . . . . 40

3.2.1 Flow Measurements in Microfluidic Devices . . . . . . . . . . . . 42

3.3 Data Analysis . . . . . . . . . . . . . . . . . . . 42

3.3.1 Calculation of Correlation Curves . . . . . . . . . . . . . . . 42

3.3 .2 Data Fitting . . . . . . . . . . . . . . . . . 43

3.3.3 Error Estimation for FCS Measurements . . . . . . . . . . . . . . 43

4 Results and Discussion $\quad 45$

4.1 Rotational Diffusion Measurements for Size and Shape Determination of Al-

dolase ............................. 45

4.2 Diffusion of Aldolase in Crowded Environments . . . . . . . . . . . . . . . 48 
4.3 Translational and Rotational Diffusion of alphaB-Crystallin . . . . . . . . . 53

4.4 Measurement of Liquid Crystal Flow Profiles in Microfluidic Channels . . . . . 55

5 Conclusions $\quad 61$

$\begin{array}{ll}\text { Acronyms } & 63\end{array}$

$\begin{array}{ll}\text { Bibliography } & 73\end{array}$

$\begin{array}{ll}\text { Acknowledgments } & 75\end{array}$ 


\section{Abstract}

The physics of fluids on microscopic lengthscales is fundamentally different from the one governing macroscopic fluid phenomena. On lengthscales up to a few micrometer, inertial forces of small particles and fluid elements are much weaker than friction and thermal forces. Consequently, fluids on microscopic length scales are characterized by laminar flow and diffusive mixing. This is of particular importance for miniaturized technical applications, such as microfluidic devices, and many biological systems. However, the exact behavior of many of theses systems is not yet fully understood and difficult to predict, especially for complicated geometries or fluids containing large amounts of solutes and macromolecules (crowded solutions). Experimental methods for measuring diffusion and flow on microscopic lengthscales can significantly increase our knowedge on these systems. Fluorescence Correlation Spectroscopy (FCS), for example, can be used for measuring translational diffusion, rotational diffusion, and flow of small fluorescent particles or molecules in solution.

Two model systems were investigated here, to study the physics of crowded solutions, using recently developed FCS approaches for rotational and translational diffusion measurements. The translational and rotational diffusion of the large protein aldolase was studied in Polyethylene Glycol (PEG) solutions for different PEG sizes and concentrations. For comparison, the macroscopic vicosity and the diffusion of small dye molecules was investigated in the same system. While all measurements indicated a similar dependence of the diffusion coefficients on PEG concentration, the extent of the reduction in the diffusion coefficients varied depending on the size of the molecule under investigation. The translational diffusion of small dyes, as well as the rotational diffusion of aldolase was less affected by an increase in PEG concentration than the macroscopic viscosity and translational diffusion of aldolase. As a second model system, the translational and rotational diffusion of the protein $\alpha \mathrm{B}$ crystallin was investigated at concentrations up to $300 \mathrm{mg} / \mathrm{ml}$. Crystallin showed a decrease in diffusion coefficients with increasing concentrations, similar to the one observed in the systems containing PEG as a crowder. Finally, flow profiles of aligned liquid crystals in microfluidic devices were studied using FCS. Importantly, a local increase in flow velocity could be observed around a defect structure in the liquid crystal for the first time. These results confirm the suitability of FCS for studying complicated crowded enviroments and microfluidic geometries, thereby contributing to a better understanding of these systems. 



\section{Introduction}

The physics of fluids at small length scales, of a few micrometer and below, is fundamentally different from the behavior of fluids that can be observed on a day to day basis in the macroscopic world. While a stone will sink immediately to the bottom of a pond, a small particle of a few nanometer in size with the same density can stay suspended in solution for a very long time. Another example is the flow and mixing behavior of fluids. While most fluids exhibit turbulent flow on the macroscopic scale, the flow at microscopic length scales is laminar, that is without turbulence. The reason for these different behaviors is the difference in the ratio of forces acting on a particle or fluid element. On macroscopic length scales the inertial forces are usually larger than friction forces, while the reverse is true for microscopic length scales.

A second fundamental difference between these two regimes is the influence of thermal motion on solute particles and fluids. On the microscopic scale, the constant collisions with solvent molecules are strong enough to move small particles and solutes around. In fact, these collisions are the reason why small particles in solution stay suspended and do not sink to the bottom. As the collisions are random in their direction and magnitude, the resulting movement of the suspended particles is also undirected. This movement of particles through a solution is called diffusion or Brownian motion. At microscopic length scales it is the only source for mixing of fluids or dissolved particles, as there are no turbulences. On the macroscopic scale, thermal collisions are usually not strong enough to move objects around, thus the stone stays at the bottom of the pond.

The physics at the microscopic length scale leads to a large number of fascinating phenomena in natural systems and technical applications. For example, biological cells are usually limited to a few micrometer in size. Therefore, the movement of a single cell in solution, such as bacteria in an aqueous environment is governed by the dominating influence of friction and laminar flow. As a result, bacteria need to employ a fundamentally different technique of propelling themselves forward than larger organisms ${ }^{[88]}$. Analogous to this, the processes inside a cell are are also dominated by friction and laminar flow. Survival of a cell depends crucially on the transport of small molecules, such as metabolites and second messengers, as well as proteins and other macromolecules. In many cases the distribution of these molecules throughout the cell is driven purely by diffusion. However, the speed of diffusive transport is limited by the size of the diffusing molecule and the viscosity of the surrounding medium. The intracellular environment is an especially interesting system, as it is crowded with a large amount of proteins, filaments, membranes and organelles (the crowders). Typical illustrations of the cell interior (fig. 1.1) might lead to the assumption that the densely crowded interior severely hinders the diffusion of biomolecules. This would have a strong influence on the speed of cellular processes such as metabolism, signaling and transport, which rely strongly on diffusion. 
However, theoretical and experimental studies have shown that this is not the case ${ }^{[100,27]}$. Especially small molecules seem to readily find gaps in crowded solutions, through which they diffuse much faster than they would through a homogeneous fluid with similar viscosity. Similar results have been obtained for in vitro measurements of diffusion in polymer solutions ${ }^{[99,85]}$, a typical model system for molecular crowding.

Despite intensive studies, the complex behavior of molecular diffusion in crowded solutions is not yet completely understood. Many theoretical simulations have predicted behavior different from the one observed in experimental studies ${ }^{[27]}$. Furthermore, a second aspect of diffusion, the rotation of molecules, has been studied only sparsely in crowded solutions and never in direct comparison with translational diffusion.

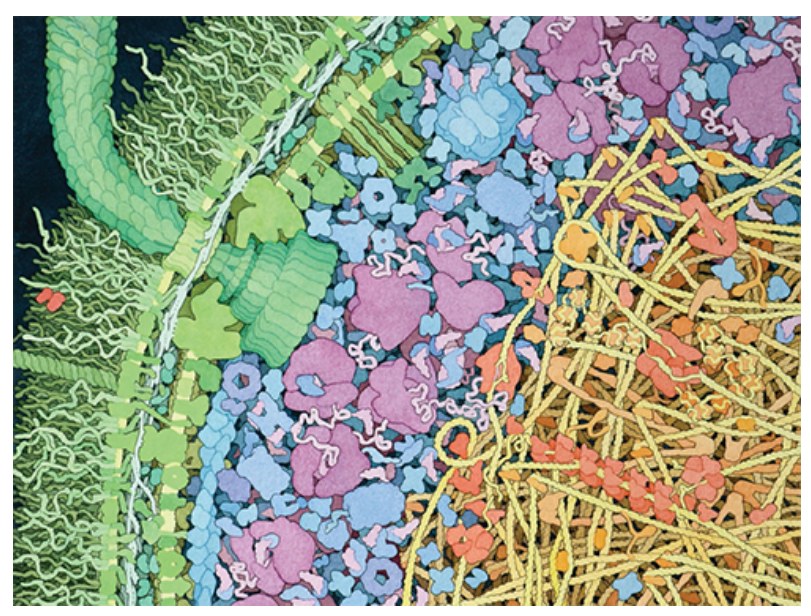

Figure 1.1: Illustration of a part of the interior of an Escherichia coli cell, showing the cell wall in green, cytoplasm in blue and purple and nucleoid region in yellow and orange (from ref. [41]).

Not only natural systems are subject to the special constraints of diffusion-limited transport and laminar flow, but also many technical applications. Fluid flow and mixing in microcapillaries or other processes that act on very small fluid volumes are limited by the same constrains as the processes inside a cell. In recent years, the search for small and efficient devices for liquid-based chemical and biological analysis has led to the emergence of the field of microfluidics ${ }^{[42,109,16,96]}$. Many of these new devices employ the properties of laminar flow, such as flow focusing devices ${ }^{[105,114]}$ or fast mixers ${ }^{[13,61]}$. Figure 1.2 shows the laminar flow around a cylindrical obstacle in a typical microfluidic channel with a rectangular profile.

Often simulations of the flow inside a microfluidic chip are used during development of new devices $^{[42]}$. However, accurate techniques for measuring the actual flow rates are necessary for fine-tuning of a design, or when complex fluids are used, which are difficult to simulate.

Within the scope of this thesis a set of techniques, based on Fluorescence Correlation Spectroscopy (FCS), is presented, which can be used for studying many of the microscopic systems mentioned above. With FCS, the fluorescence intensity fluctuations from a small sample volume can be analyzed, to measure molecular diffusion, size, or interactions ${ }^{[74,32,91,63,40]}$. In this 


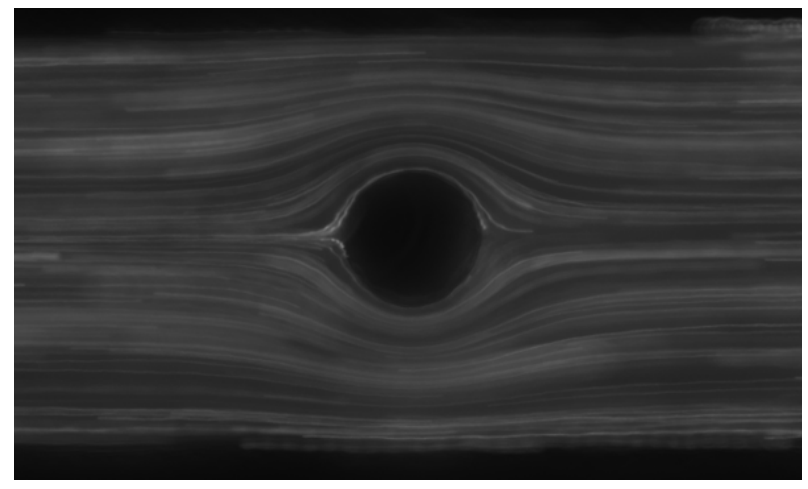

Figure 1.2: Fluorescence image of the laminar flow around a pillar in a microfluidic device. The image shows the traces of small fluorescent spheres visualized by a long exposure time of the image.

work FCS will be used for rotational diffusion measurements ${ }^{[71,86]}$ to accurately determine the size of the macromolecule aldolase and even to gain some information about its shape. If the size of a molecule is known, it can be used for accurately determining the viscosity of a sample solution. This technique is applied in conjunction with Dual Focus Fluorescence Correlation Spectroscopy (2fFCS) ${ }^{[24]}$, a method for accurately determining the translational diffusion of molecules.

It is shown here that the combination of FCS for measuring rotational diffusion and 2fFCS for measuring translational diffusion is ideal for studying crowded environments. The translational and rotational diffusion of molecules in crowded solutions is systematically studied for varying crowder concentrations in two different model systems. First, the diffusion of small dyes and macromolecules in crowded Polyethylene Glycol (PEG) solutions is analyzed. In comparison, the diffusion of $\alpha \mathrm{B}$-crystallin $(\alpha \mathrm{B})$, a small heat shock protein, is analyzed in highly concentrated $\alpha \mathrm{B}$ solutions. In the last part, the utility of 2fFCS for measuring flow velocities in microfluidic channels is demonstrated. The flow velocity profiles of a Liquid Crystal (LC), a complex anisotropic liquid, are presented for different channel geometries.

\subsection{Introduction to Fluorescence Correlation Spectroscopy}

Fluorescence Correlation Spectroscopy (FCS) is a powerful technique for studying the diffusion, dynamics and interactions of biomolecules and other small particles in solution. It was first employed in the 1970s by Magde, Elson and Webb to study the thermodynamic fluctuations in the binding of Ethidium Bromide $(\mathrm{EtBr})$ to $\mathrm{DNA}^{[74]}$. In their original experiment a laser beam was focused into a sample solution to excite the fluorescence of the EtBr molecules. The emitted light was collected with a parabolic collector, filtered to reject any stray laser light, and detected with a photomultiplier tube (see fig. 1.3).

As the fluorescence intensity of $\mathrm{EtBr}$ is enhanced upon binding to $\mathrm{DNA}^{[84]}$, binding and 
unbinding of EtBr leads to intensity fluctuations in the detected fluorescence signal. The frequency of the intensity fluctuations is characteristic for the binding kinetics of EtBr to DNA. These fluctuations can be quantified easily by calculating the autocorrelation of the fluorescence signal. The actual rate constants of the binding reaction are obtained from fitting the calculated correlation curves with an appropriate model function. The binding of EtBr to DNA, for example, is well described by single-step binding kinetics.

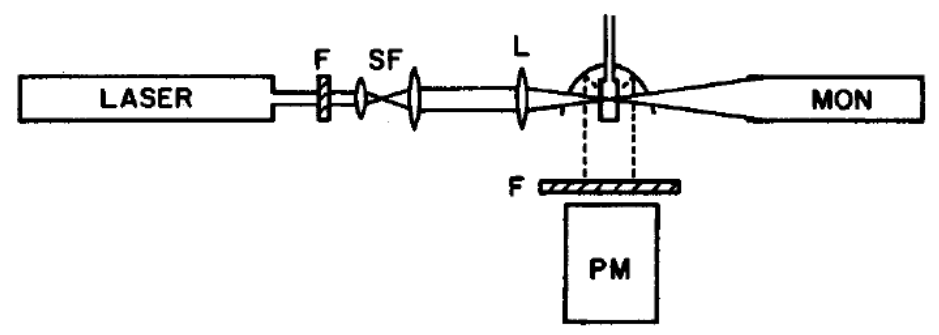

Figure 1.3: Schematic of the setup used for the FCS experiments by Magde et al.. The symbols are: F, colored filter; SF, spatial filter; L, lens; MON, laser intensity monitor; PM, photomultiplier. The sample cell and the parabolic fluorescence-collecting mirror are also shown. (from Ref. [75])

Besides intensity fluctuations of the dye molecules themselves, like the ones upon binding of ethidium bromide to DNA, any other source of intensity fluctuations can be studied with fluorescence correlation spectroscopy. One additional source of fluctuations is the number of fluorescent particles in the detection volume, the volume from which fluorescence is recorded. There will be a proportional change in fluorescence intensity whenever the number of particles in the detection volume changes. If the FCS measurement is taken only in a small part of sample solution, the particle concentration will constantly fluctuate around its average value due to the diffusion of particles in and out of this detection volume. For a known size and shape of the detection volume, it is therefore possible to determine the diffusion coefficient of the fluorescent particle or molecule from the autocorrelation of the fluorescence intensity ${ }^{[32,75]}$. The diffusion of molecules or particles with little or no fluorescence can be measured by attaching a small fluorescent label.

\subsection{Molecular Crowding}

Studying the diffusion of proteins and small biomolecules in their native environment is of particular importance for understanding many biological functions. The diffusion of small molecules, so called morphogens, plays a crucial role during cellular differentiation and embryonic development ${ }^{[44,68,21]}$. Furthermore, throughout the whole life of a cell, diffusion of small messenger molecules ${ }^{[55]}$, metabolites ${ }^{[39]}$ and proteins is of great importance. However, the diffusion of small molecules in such complex and crowded environments, as the cell cytoplasm or intra-organellar compartments, can not be described readily by the macroscopic viscosity of these solutions ${ }^{[99]}$. For homogeneous fluids, the dependence of the diffusion speed 
on particle size and the viscosity is given by the Stokes-Einstein relation, which will be discussed later in more detail. However, for crowded solutions the Stokes-Einstein relation has to be used with caution.

To be more precise, the concept of a continuum viscosity is not directly applicable to the diffusion of small molecules through a crowded solution. The local environment of a molecule smaller than the crowder is far from homogeneous. On the one hand, for very short distances, comparable to their own size, the environment is mostly dominated by the interactions with solvent molecules, e.g. water. On the other hand, on longer distances, diffusion should be influenced more strongly by the crowding molecules. However, small molecules still find gaps between the larger molecules through which they can diffuse much faster than larger molecules could. This inhomogeneity in the local environment should have an even stronger influence on the rotation of small molecules, provided that the interactions with the crowders are not of an attractive (sticky) nature. In case of repulsive interactions, rotation should be influenced only litle by the other molecules in the solution, as it is not connected to a translational motion through the crowded solution. In support of this idea, the rotation times of a small fluorescent dye inside fibroblasts measured by fluorescence anisotropy were found to be close to those observed in free solution ${ }^{[38]}$. This is indicative of mainly repulsive interactions between the dye and other molecules and proteins in the cell cytoplasm, and of only a minor contribution of hydrodynamic interactions.

For macromolecules, however, the picture might be completely different. Larger molecules do not easily find gaps between other large molecules in crowded solutions. Furthermore, hydrodynamic interactions may have a stronger influence on rotational and translational diffusion of macromolecules. Indeed, recent studies using Brownian Dynamics simulations identified hydrodynamic interactions as a major influence on macromolecular motion ${ }^{[3]}$. In agreement with these simulations, experimental measurements in vitro showed a strong dependence of diffusive behavior on the size of the diffusing molecule, the size of the crowder and its concentration $^{[50]}$. These results also showed that the Stokes-Einstein relation can be used in these systems as long as an effective and size-dependent viscosity is used. Overall, the effect of crowding on the behavior of macromolecules is still not completely understood. Particularly, its influence on processes other than diffusion, such as binding ${ }^{[22]}$ or folding ${ }^{[59]}$ of proteins is still poorly understood. This work expands the methodology of measuring diffusion in crowded solutions and contributes to the understanding of the molecular interactions in these systems.

The techniques used so far for the study of molecular crowding include Fluorescence Recovery After Photobleaching (FRAP) ${ }^{[54]}$, Single Particle Tracking (SPT) ${ }^{[53]}$, capillary electrophoresis $^{[89]}$ and conventional $\mathrm{FCS}^{[50]}$. FRAP studies have the disadvantage of being limited in the accessible time range. Furthermore, recovery curves are difficult to interpret if different diffusing species are present. In addition, recovery from photo-physical dark states such as the triplet-state can confuse the diffusion measurements. SPT is similarly restricted by its short measurement range. Tracking of single particles for longer times can be difficult in three dimensional samples, as particles will diffuse in and out of the focal plane. Of all the methods available, FCS is the one with the highest dynamic range and accuracy. 
Rotational diffusion in crowded solutions has so far been studied only by fluorescence anisotropy decay ${ }^{[38]}$. However, fluorescence anisotropy of most fluorescent dyes is limited to measuring on very short time ranges. Even though longer time ranges are in principle accessible to phosphorescence anisotropy decay, little work has been done in this direction. So far, comparative experimental studies of translational and rotational diffusion of macromolecules in crowded environments are missing. Altogether, fluorescence correlation spectroscopy offers an attractive alternative to measure rotational and translational diffusion over a broad time range and is also applicable to crowded solutions. This work contributes to a better understanding of diffusion in crowded solutions by systematically investigating both the rotational and the translational diffusion in two systems with molecular crowding.

\subsection{Microfluidics}

At first glance, microfluidics may seem a completely different field of research than molecular crowding. Upon closer inspection, however, a number of striking similarities are revealed. As mentioned above, the flow of fluids in small channels, such as those used in microfluidics, is free of turbulences, thus resembling the situation in biological systems. Moreover, the mixing of fluids and solutes in microfluidic devices is solely determined by diffusion, like many processes inside cells. Finally, one main application of microfluidic devices is the analysis of biological samples, such as blood or cell extracts. Therefore, a good understanding of the behavior of complex fluids inside microfluidic devices will help to advance the development of such applications.

The search for small and efficient devices for biological and chemical analysis leads to the development of advanced microfluidic devices. Just as computer chips have undergone rapid development over the last decades, instruments for bioanalytical and chemical research have undergone constant evolution. However, their development and miniaturization is not nearly as fast as that of electronic devices. True miniaturization would have the potential to revolutionize research in the life sciences, medical and chemical analysis. The ultimate goal is to perform a complex multiparameter analysis only from a minimum volume of sample loaded on a single small chip or device. The reduced amount of reagents needed for such a miniaturized analysis alone would be an enormous cost benefit. In addition, it would be of great practical ease for medical tests if sample purification and a routinely used diagnostic method, such as the Polymerase Chain Reaction, could be integrated on a small, cheap and disposable chip. Furthermore, such devices could be easily parallelized for high throughput assays if they are sufficiently small and cheap. In biochemical and pharmaceutical research these chips could significantly decrease the time needed for complex screens.

Pursuing the construction of these novel devices has been one of the driving forces of microfluidic research. Microfluidics uses small channels with a width of only tens to hundreds of micrometers, as well as specially structured regions for processing small fluid volumes ${ }^{[42,109,16,96]}$. The first microfluidic devices had channels which were etched into silicon or glass, whereas 
newer devices often use elastomer chips produced from Polydimethylsiloxane (PDMS) with the help of negative molds (see Fig. 1.4). Recent applications of microfluidic chips include fast integrated immunoblotting ${ }^{[48]}$, reliable mixing of sub-attoliter volumes ${ }^{[18]}$ and sample analysis in disease diagnostics ${ }^{[17]}$. Many microfluidic devices still need an external detector such as a microscope for analysis as well as a flow or pressure controller to regulate flow in the different channels. The incorporation of optical detection into the microfluidic chip lead to the development of the so called optofluidic chips ${ }^{[87]}$, the combination of microfluidics and optics.

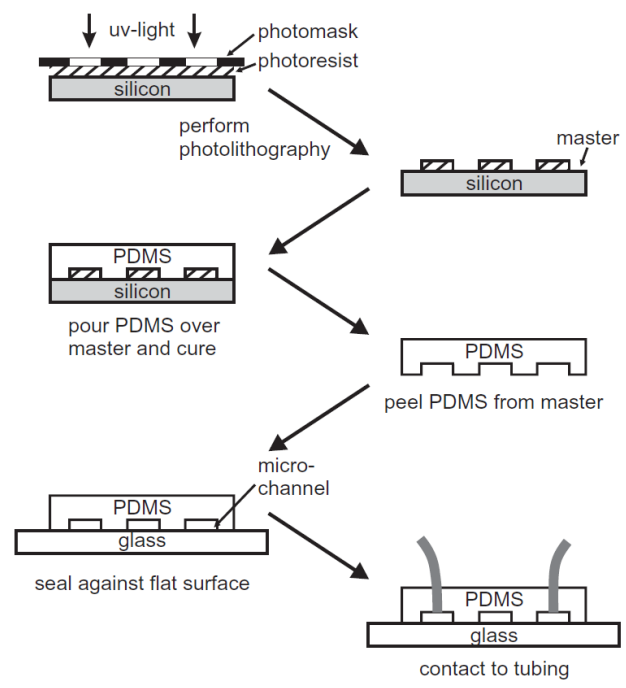

(a)

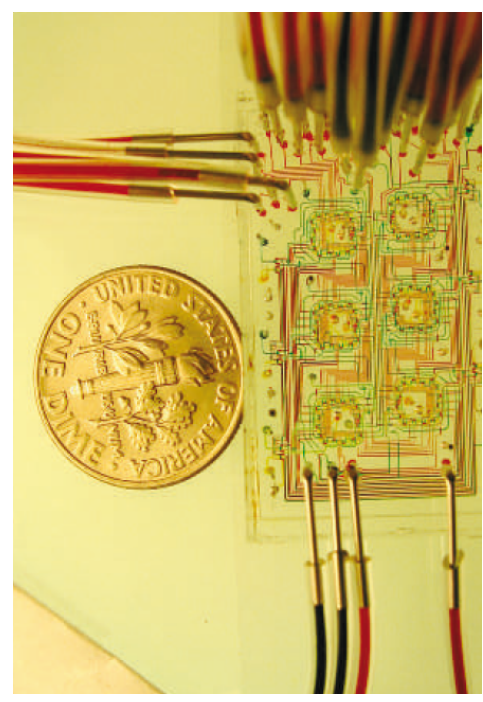

(b)

Figure 1.4: (a): Typical steps of microfluidic device preparation using soft lithography (from ref. [96]). (b): An example microfluidic device for monitoring bacterial growth (from ref. [9]).

The successful development of new microfluidic devices requires extensive knowledge of the flow behavior of liquids and emulsions confined to such small volumes. Even though the flow inside the channels of a new chip is commonly simulated during development ${ }^{[42,20]}$, the accurate measurement of actual flow profiles in a chip is indispensable for complicated geometries or for fine-tuning a design. Today, several methods are available for measuring the flow in microfluidic channels. Among the most popular techniques are Particle Image Velocimetry (PIV) ${ }^{[95,79,1]}$, UV photo-activation ${ }^{[69]}$, Fluorescence Photobleaching ${ }^{[104]}$ and FCS $^{[43,66]}$.

PIV is frequently chosen because of its simplicity. PIV requires the addition of small fluorescent particles or particles with high contrast to the solution in the microfluidic device. A video recorded with a camera on a standard (fluorescence) microscope can be used to analyze the flow velocities in the device. However, the presence of such tracer particles, which might be as large as several micrometers, can already disturb the flow significantly. Furthermore, the distribution of these particles in mixing or flow focusing geometries does not accurately represent the distribution of small soluble molecules, due to their different diffusion time. Finally, the spatial resolution is limited, especially along the optical axis (z-direction). 
UV photo-activation and fluorescence bleaching rely on the uncaging or bleaching of small fluorescent dyes. The fluorescent dyes are typically only $0.5-1 \mathrm{~nm}$ in diameter and disturb the flow much less than the larger fluorescent particles. Due to their faster diffusion the dyes spread more evenly. Nonetheless, these techniques usually use an epi-fluorescence microscope and are therefore also limited in z-resolution.

Compared to this, FCS can readily achieve a spatial resolution similar to that of confocal imaging, as the confocal detection allows for lateral resolution of approximately $0.5 \mu \mathrm{m}$ and axial resolution of $2 \mu \mathrm{m}$. Fluorescence correlation spectroscopy should therefore be the method of choice whenever superior accuracy and true three-dimensional flow profiling is desired.

Despite that, problems with FCS measurements can arise when fluids other than aqueous solutions are used in the microfluidic chip. Many microfluidic devices use oil phases to create small water droplets as reaction containers ${ }^{[96]}$. An interesting and relatively new research field involves the use of molecularly ordered fluids, namely liquid crystals, in microfluidic devices $^{[97]}$. In this case the optical properties of the fluid are very different from those of typical fluorescence microscopy samples. The corrections of standard microscopy objectives are insufficient, and consequently the focus is distorted. The uncertainty in knowing the size and shape of the detection volume of the FCS set up makes accurate absolute measurements of flow velocity in oil or liquid crystal phases difficult. This difficulty was circumvented in non-flow FCS by the introduction of an external ruler with the use of Dual Focus Fluorescence Correlation Spectroscopy $(2 \mathrm{fFCS})^{[24]}$. This technique can be used for accurate flow measurements in microfluidic devices if the displacement of the two foci is along the direction of the flow ${ }^{[6]}$. In this way, the problems arising from mismatched optics are overcome, even when measuring in complex solvents like liquid crystals.

\subsection{Nematic Liquid Crystals}

Nematic liquid crystals are molecules in an intermediate phase that have fluidic properties, while at the same time retaining a long ranged molecular ordering similar to that of a crys$\operatorname{tal}^{[23,19]}$. They are typically rod-like molecules that spontaneously align their long axis in parallel, resulting in large aligned patches (fig. 1.5a). However, different regions might still have different directions of alignment leading to defect structures at the interfaces between aligned regions. A defect is a point or line along which no distinct molecular orientation can be identified. This can nicely be seen in schlieren images ${ }^{[26]}$, which make the differences in optical path length in a medium visible. Such an image which shows the defects between the different domains is depicted in figure $1.5 \mathrm{~b}$.

The optical and fluidic properties of liquid crystals can strongly be influenced by externally applied forces and the shape of the container. Most commonly, a flow field or external electrical field is applied to change the properties of the liquid crystal. Additionally, the walls of the container can be treated to favor a specific alignment, a modification known as surface anchoring. Also, defined patterns of alignment can be generated in a confined container with specific surface anchoring and the application of an external field. For example, the confinement to a capillary with homeotropic surface anchoring (alignment perpendicular to the surface) can lead 


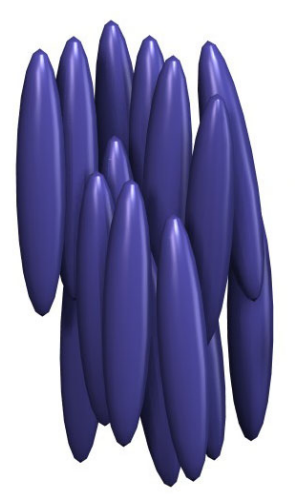

(a)

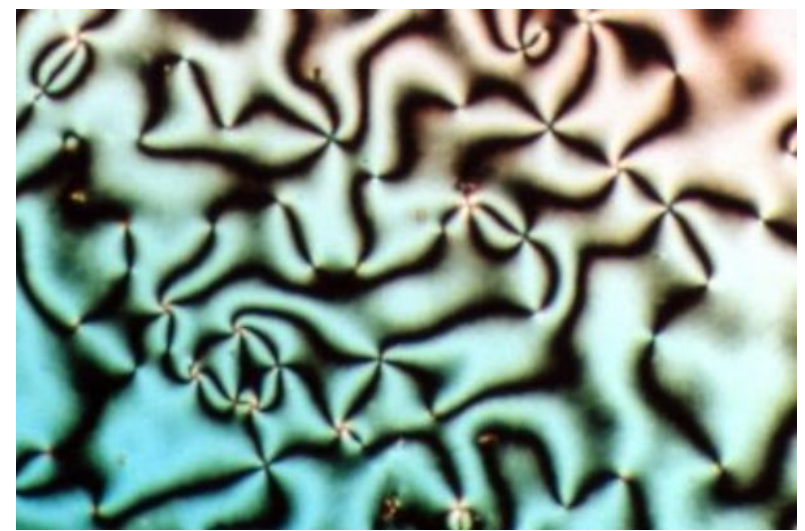

(b)

Figure 1.5: (a): Alignment of the molecules in a nematic liquid crystal. (b): Schlieren image of a liquid crystal in nematic phase showing defects. (from Wikimedia Commons ${ }^{[37]}$ )

to line or ring defects ${ }^{[73]}$. These defects can spread over a long range through the container. The Planar Radial with Line Defect (PRLD) (fig. 1.6a) and the Escape Radial (ER) (fig. 1.6b) are commonly generated defects.

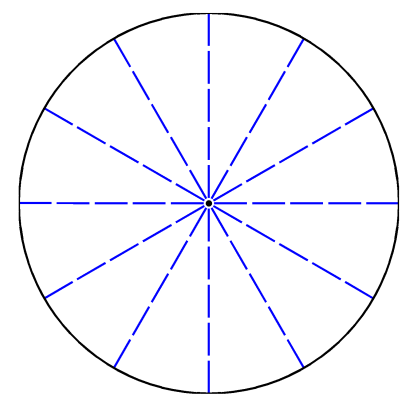

(a)

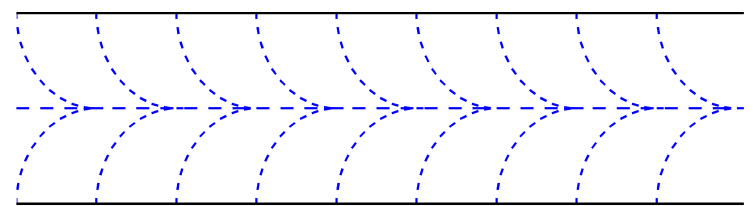

(b)

Figure 1.6: Ordering structures of nematic liquid crystals in micro-capillaries with homeotropic surface anchoring. (a): Cross section of a capillary with planar radial alignment with a line defect in the center; (b): longitudinal view of a capillary with radial escape alignment.

The best known application of liquid crystals is their use in liquid crystal displays, where an external electrical field is used to influence the optical properties of the liquid crystal. The study of liquid crystals in capillaries is of particular interest since they constitute the building units of specialized high-performance fibers such as carbon fibers or spider's silk, produced from nematic precursors ${ }^{[73,78,64]}$. In the future, other exciting applications for microfluidic devices combined with liquid crystals might emerge. Some of which might include their use in optofluidics and reconfigurable photonic devices ${ }^{[81]}$, or for specialized functions in dropletmicrofluides ${ }^{[46,96]}$. 
In this work, a special microfluidic geometry is used to stabilize an ER defect in a nematic liquid crystal at low flow velocities. For the first time the flow profile on a line through such a defect could be measured with $2 \mathrm{fFCS}$. 


\section{Theoretical Background}

\subsection{Theory of Diffusion}

Molecular diffusion is the random thermal motion of a particle, molecule or atom in a gas or liquid. This random motion is the reason for phenomena such as a gas spreading out equally to fill a container, or an ink drop spreading in a glass of water. The rate of diffusion of particles in solution is dependent on the temperature, the viscosity of the solution and the size of the particle.

One of the first scientists to observe diffusion of single particles was Robert Brown, who observed the random movements of pollen grains under a microscope in 1827. The diffusion of small particles in a solution is therefore often referred to as Brownian motion. However, one has to keep in mind that the solute molecules also undergo constant diffusion, known as self-diffusion.

Molecules diffusing in solution do not keep one orientation, but constantly undergo random rotations. These small rotations are similar to the random steps of displacement in translational diffusion. Both types of random motion originate from the constant collisions with the solvent molecules. These collisions occur randomly leading to rotation as well as displacement of the molecules. The overall movement of the molecule can be separated into the movement of its center of mass (translational diffusion) and the rotation around its three principal axes (rotational diffusion). If the position of a single molecule is recorded over time, its trajectory looks like a random walk, with varying step sizes and directions (fig. 2.1a). The rotational diffusion can be visualized by plotting the intersection of any fixed vector through the molecule with the unit sphere over time. The resulting trajectory will be a random walk on the surface of the unit sphere, representing the molecular rotation (fig. 2.1b).

\subsubsection{Translational Diffusion}

The trajectory of a single particle, diffusing through a solution, is impossible to predict due to the large number of collisions with the surrounding solvent molecules that occur constantly. However, statistical predictions about the position of the particle can be made. For large particle numbers this leads to the deterministic macroscopic behavior, like the spreading of a drop of ink in water mentioned above. More generally, there will always be a net flux of molecules from a region of higher concentration to a region of lower concentration. It was discovered by Adolf Fick in 1855, that this flux $j$ is proportional to the gradient of the concentration $c$, in the way 


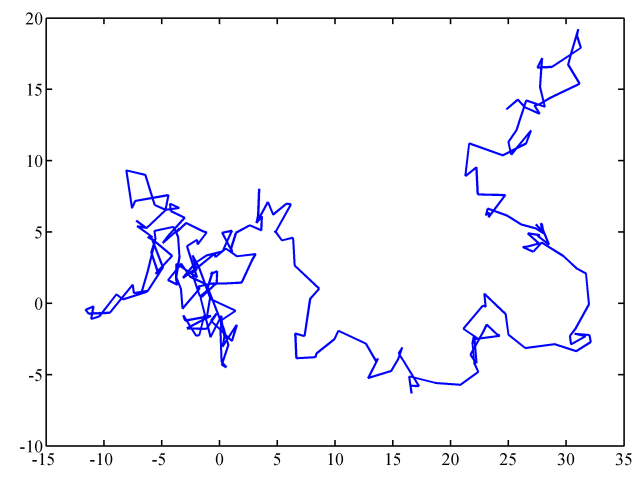

(a)

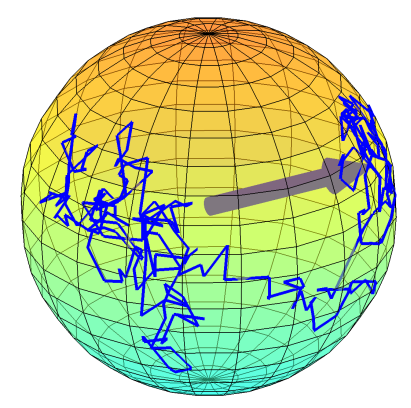

(b)

Figure 2.1: (a): Translational diffusion of a molecule in two dimensions (arbitrary units). (b): Rotational diffusion of a molecule around two axes, visualized by the diffusion on the surface of a three dimensional sphere. The orientation of the molecule is given by the vector from the center of the sphere to the current position on the surface (gray arrow).

$$
j=-D \nabla c
$$

where the proportionality $D$ is called the diffusion constant. The change of concentration in one small volume element of the sample is, for non-reacting molecules, solely determined by the flux in and out of this volume:

$$
\frac{\partial c}{\partial t}=-\nabla j
$$

Inserting 2.1 into 2.2 one obtains the diffusion equation in three dimensions:

$$
\frac{\partial c}{\partial t}=\nabla(D \nabla c)
$$

The probability for a single molecule to move from a position $\mathbf{r}_{0}$ to a position $\mathbf{r}_{1}$ within time $\tau$ is given by Green's function of the above equation:

$$
G\left(\mathbf{r}_{1}-\mathbf{r}_{0}, \tau\right)=\frac{1}{(4 \pi D \tau)^{3 / 2}} \exp \left[-\frac{\left(\mathbf{r}_{1}-\mathbf{r}_{0}\right)^{2}}{4 D \tau}\right]
$$

which is the solution of the diffusion equation for the initial condition

$$
\lim _{\tau \rightarrow 0} G\left(\mathbf{r}_{1}-\mathbf{r}_{0}, \tau\right)=\delta\left(\mathbf{r}_{1}-\mathbf{r}_{0}\right)
$$

(see fig. 2.2).

This expression allows to calculate the moments of the diffusive movement:

$$
\langle\mathbf{r}(t)\rangle=\int \mathbf{r} G(\mathbf{r}, t) d^{3} \mathbf{r}=0
$$

and 


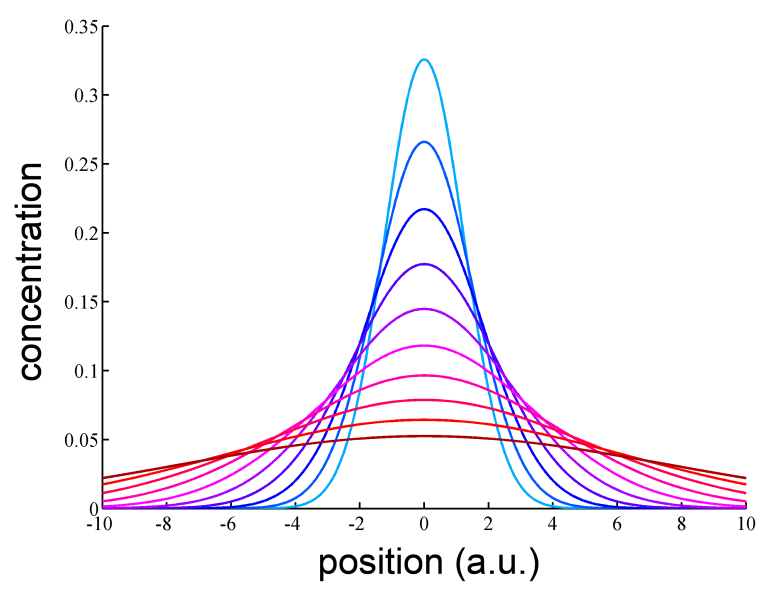

Figure 2.2: Solution to the diffusion equation in one dimension (increasing time from from light blue to dark red curve).

$$
\left\langle\mathbf{r}^{2}(t)\right\rangle=\int \mathbf{r}^{2} G(\mathbf{r}, t) d^{3} \mathbf{r}=6 D t
$$

The first moment is zero, as the probability of moving in any direction is the same. However, the mean square displacement increases linearly with time.

The question arises how large the diffusion coefficient of a small particle is. This can be determined by considering the diffusion of particles under the influence of an external force. Suppose the particle is subject to the gravitational force $F=m g$, where $m$ is the mass of the particle and $g$ the gravitational acceleration. In solution a particle will quickly acquire the speed

$$
v=\mu m g
$$

where $\mu$ is the mobility of the particle. The mobility of a spherical particle was found by Stokes to be $\mu=1 /(6 \pi \eta r)$, where $\eta$ is the dynamic viscosity of the solution. As a consequence, there will be a resulting downward flux of particles proportional to the concentration in the solution:

$$
j_{\text {drift }}(x)=-\mu m g c(x) .
$$

In equilibrium, this must be balanced by a diffusive flux (eq. 2.1). Furthermore, the particles will be distributed according to the barometric distribution

$$
c(x)=c_{0} e^{-m g x / k_{B} T},
$$

where $k_{B}$ is the Boltzmann constant and $T$ the temperature of the solution. Hence, equilibrium requires: 


$$
\begin{aligned}
0 & =j_{\text {drift }}+j_{\text {diffusion }}=-\mu m g c(x)-D \frac{d}{d x} c(x) \\
& =-c(x)\left(\mu m g-D \frac{m g}{k_{B} T}\right)
\end{aligned}
$$

The diffusion coefficient can then be expressed as:

$$
D=\mu k_{B} T=\frac{k_{B} T}{6 \pi \eta r}
$$

which is known as the Stokes-Einstein relation. This relation allows to calculate the diffusion coefficient of a spherical particle with known radius in a solution of known viscosity. It also can be used to determine the size of a particle if the diffusion coefficient is known. For non-spherical particles one can calculate the hydrodynamic radius, the radius of an equivalent sphere with the same diffusion coefficient.

\subsubsection{Rotational Diffusion}

Since actual particles and molecules are not ideal point particles, they have internal degrees of freedom like vibration and rotation. These degrees of freedom also exchange energy through molecular interactions. Hence, in addition to translational diffusion, particles in solution undergo rotational diffusion as well.

The general theory of rotational diffusion in three-dimensional space is more complicated than that of translational diffusion, but is mathematically equivalent to the treatment of a quantum-mechanical rotor, which is a well studied subject. In the context of FCS it was first addressed by Aragón and Pecora ${ }^{[5,11]}$ and was first adapted to modern single photon-counting detection schemes by Loman et al. ${ }^{[71]}$. A good starting point is the rotational diffusion equation

$$
\frac{\partial P}{\partial t}=\left(D_{a} \hat{J}_{a}^{2}+D_{b} \hat{J}_{b}^{2}+D_{c} \hat{J}_{c}^{2}\right) P
$$

where $a, b$, and $c$ denote the principal axis of rotation of the molecule, $P=P(\psi, \theta, \phi)$ is the probability density of finding the molecule's principal axes rotated by Euler angles $\psi, \theta$, and $\phi$ with respect to the laboratory frame, the $D_{a, b, c}$ are the diffusion coefficients for rotation around there respective principal axis, and the $\hat{J}_{a, b, c}$ are the angular momentum operators around these axes. Equation 2.13 can be derived analogously to the translational diffusion equation 2.3. One difficulty with eq. 2.13 is that the angular momentum operators relate to the intrinsic frame of the molecule's principal axes which is rotating in time with respect to the fixed lab frame. To simplify matters, one can first rotate the molecule back to the lab's frame so that its axes align with the fixed Cartesian coordinate axes of the lab frame, then apply the operator, and finally rotate the molecule back, i.e.

$$
\frac{\partial P}{\partial t}=\hat{R}\left(D_{a} \hat{J}_{x}^{2}+D_{b} \hat{J}_{y}^{2}+D_{c} \hat{J}_{z}^{2}\right) \hat{R}^{-1} P
$$


where $\hat{R}$ denotes the operation of rotating the molecule's frame from an orientation aligned with the lab's Cartesian $x, y, z$-coordinates to its actual orientation as specified by the Euler angles $\psi, \theta$, and $\phi$, see (fig. 2.3).

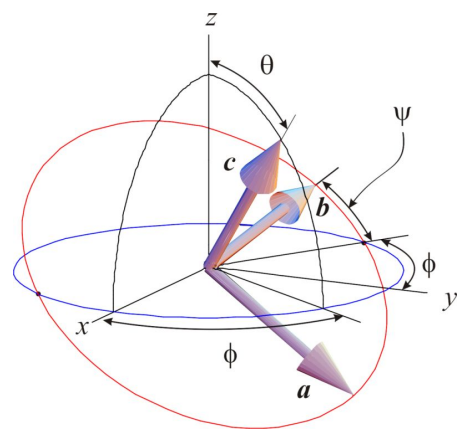

Figure 2.3: Geometric meaning of the three Euler angles $\psi, \theta$, and $\phi$. Shown are the molecule's three principal (and orthogonal) axes of rotation $a, b$, and $c$, and the three Cartesian axes $x, y$, and $z$ of the lab frame.

The rotation operator $\hat{R}$ can be decomposed into

$$
\hat{R}=\hat{R}_{z}(\phi) \hat{R}_{y}(\theta) \hat{R}_{x}(\psi)
$$

where $\hat{R}_{y, z}(\beta)$ denotes a rotation by angle $\beta$ around axis $y$ or $z$, respectively. The advantage of eq. 2.14 is that the angular momentum operators are now referring to the fixed lab frame.

If one can find a complete set of Eigenfunctions of eq. 2.14 one can solve the rotational diffusion equation using the Green's function in the standard way. For this purpose the function $P$ can be replaced by the special case

$$
P_{l m}=\hat{R}|\ell, m\rangle \cdot c(t)
$$

where $|\ell, m\rangle$ is an eigenfunction of the angular momentum operator obeying the two relations

$$
\begin{aligned}
\hat{\mathbf{J}}^{2}|\ell, m\rangle & =\left(\hat{J}_{x}^{2}+\hat{J}_{y}^{2}+\hat{J}_{z}^{2}\right)|\ell, m\rangle \\
& =\ell(\ell+1)|\ell, m\rangle
\end{aligned}
$$

and

$$
\hat{J}_{z}|\ell, m\rangle=m|\ell, m\rangle \text {. }
$$

$c(t)$ contains the time dependent part of $P_{l m}$. Any function $P$ can always be represented as superposition of the $P_{l m}$.

For the sake of simplicity, one can further only consider the special case of a symmetric top rotor where one has $D_{a}=D_{b}=D_{\perp}$ and $D_{c}=D_{\|}$. This is also the case of most practical relevance, for real FCS data will rarely allow for discerning more than two non-equal rotation axes. Inserting eq. 2.16 into eq. 2.14 then yields 


$$
\begin{aligned}
\frac{\partial c(t)}{\partial t} \hat{R}|\ell, m\rangle & =c(t) \hat{R}\left(D_{\perp} \hat{J}_{x}^{2}+D_{\perp} \hat{J}_{y}^{2}+D_{\perp \perp} \hat{J}_{z}^{2}\right)|\ell, m\rangle \\
& =c(t) \hat{R}\left(D_{\perp}\left(\hat{\mathbf{J}}^{2}-\hat{J}_{z}^{2}\right)+D_{\|} \hat{J}_{z}^{2}\right)|\ell, m\rangle \\
& =c(t)\left(D_{\perp} l(l+1)+\left(D_{\|}-D_{\perp}\right) m^{2}\right) \hat{R}|\ell, m\rangle
\end{aligned}
$$

Using an exponential function one finds the solution $c(t)=e^{-\left[D_{\perp} l(l+1)+\left(D_{\|}-D_{\perp}\right) m^{2}\right] t}$.

Next, one has to clarify how the rotation operator $\hat{R}$ acts on $|\ell, m\rangle$. One of the most elegant derivations of this action is given by Feynman ${ }^{[36]}$ using the possibility to represent any state $|\ell, m\rangle$ through a combination of spin-1/2 states for which the transformation relations under action of $\hat{R}$ are well known. The final result is:

$$
\hat{R}(\phi, \theta, \psi)|\ell, m\rangle=\sum_{k=-\ell}^{\ell} e^{i k \phi} D_{k m}^{\ell}(\phi, \theta, \psi)|\ell, k\rangle
$$

where the $D_{k m}^{l}(\phi, \theta, \psi)$ are Wigner rotation matrices defined by

$$
D_{k m}^{l}(\phi, \theta, \psi)=\exp (i k \phi+i m \psi) d_{k m}^{l}(\theta)
$$

and

$$
\begin{aligned}
& d_{k m}^{l}(\theta)=\left\langle l, k\left|R_{y}(\theta)\right| l, m\right\rangle= \\
& \frac{\sqrt{(j+k) !(j-k) !(j+m) !(j-m) !}}{k !} \\
& \times \sum_{n} \frac{(-1)^{j+k-n} C^{2 n-m-k} S^{2 j+m+k-2 n}}{(j+m-n) !(j+k-n) !(n-m-k) !}
\end{aligned}
$$

where the abbreviations $C=\cos (\theta / 2)$ and $S=\sin (\theta / 2)$ have been used.

By multiplying eq. 2.19 with $\langle\ell, k|$, one realizes that the functions

$$
e^{-\left[D_{\perp} \ell(\ell+1)+\left(D_{\|}-D_{\perp}\right) m^{2}\right] t} D_{m k}^{\ell}(\phi, \theta, \psi)
$$

are eigenfunctions of the rotational diffusion equation. Furthermore, Wigner's rotation matrices obey the orthogonality relation

$$
\begin{array}{r}
\int_{0}^{\pi} d \theta \sin \theta \int_{0}^{2 \pi} d \phi \int_{0}^{2 \pi} D_{m k}^{\ell *}(\phi, \theta, \psi) D_{m^{\prime} k^{\prime}}^{\ell^{\prime}}(\phi, \theta, \psi) \\
=\frac{8 \pi^{2}}{2 \ell+1} \delta_{\ell, \ell^{\prime}} \delta_{k, k^{\prime}} \delta_{m, m^{\prime}}
\end{array}
$$


where the star denotes complex conjugation. The $\delta_{a, b}$ are Kronecker symbols taking the value one for $a=b$ and zero otherwise. With this complete orthonormal system of eigenfunctions, the probability density that a molecule has rotated, within time $t$, from an initial orientation $\Omega^{\prime}$ described by the Euler angles $\phi^{\prime}, \theta^{\prime}$ and $\psi^{\prime}$ into a final orientation $\Omega$ described by Euler angles $\phi, \theta$ and $\psi$ is given by Green's function in the standard way as

$$
\begin{array}{r}
G\left(\Omega, \Omega^{\prime}, t\right)=\sum_{\ell=0}^{\infty} \sum_{k, m=-\ell}^{\ell} \frac{2 \ell+1}{8 \pi^{2}} D_{k m}^{\ell}(\phi, \theta, \psi) \\
\times D_{k m}^{\ell *}\left(\phi^{\prime}, \theta^{\prime}, \psi^{\prime}\right) e^{-\left[D_{\perp} \ell(\ell+1)+\left(D_{\|}-D_{\perp}\right) m^{2}\right] t}
\end{array}
$$

\subsection{FCS}

\subsubsection{Fluorescence}

Fluorescence is the emission of a photon by an atom or molecule upon the relaxation of an electron from an exited state to the ground state (fig. 2.4a). Excitation in turn occurs upon illumination with light or radiation of sufficient energy, or by other pathways such as energy transfer from another molecule or atom. The light of the emitted photons has typically a longer wavelength than that of the excitation light. This so called Stokes shift is due to the vibrational relaxation of the molecule and polar relaxation of the surrounding medium, if the medium is polarizable (fig. 2.4b).

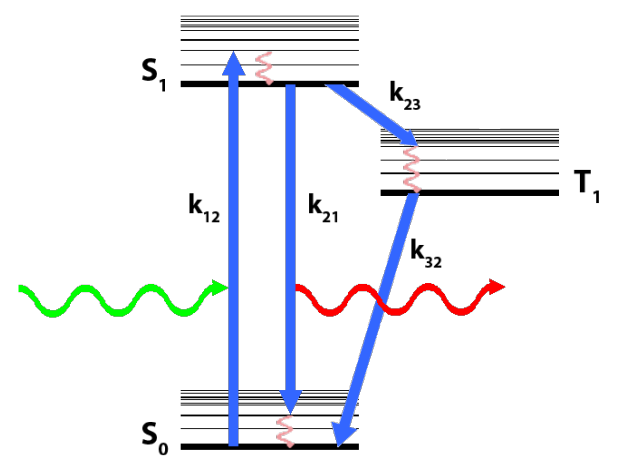

(a)

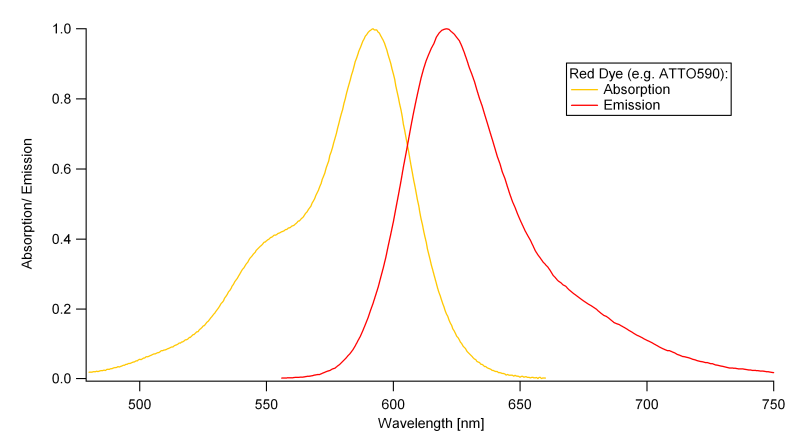

(b)

Figure 2.4: (a): Jablonski diagram, illustrating the electronic levels of a dye molecule. The molecule is excited from the ground state $S_{0}$ to the first excited state $S_{1}$ by a photon with suficient energy (green arrow). After relaxation into the vibrational ground state of $S_{1}$ the molecule relaxes to $S_{0}$, accompanied by the emission of a photon with slightly lower energy (red arrow). (b): Absorption and emission spectra of a dye emitting red light (ATTO 590).

The relaxation of a dye to its ground state is a Poisson process with a constant transition rate $k_{21}$. Therefore, the probability of the molecule for being in the excited state changes as 


$$
\frac{d}{d t} P_{\mathrm{ex}}=-k_{21} P_{\mathrm{ex}}
$$

The solution of this equation is given by

$$
P_{\mathrm{ex}}(t)=P_{\mathrm{ex}}^{0} e^{-t / \tau_{F}},
$$

where $\tau_{F}=1 / k_{21}$ is the fluorescence lifetime of the dye and $P_{\mathrm{ex}}^{0}$ is the initial probability of the dye occupying the excited state. The fluorescence intensity of a solution of dyes, emitted after an excitation pulse, is proportional to the number of dyes in the excited state. Hence, the fluorescence intensity shows an exponential decay, from which the fluorescence lifetime can be determined (fig. 2.5).

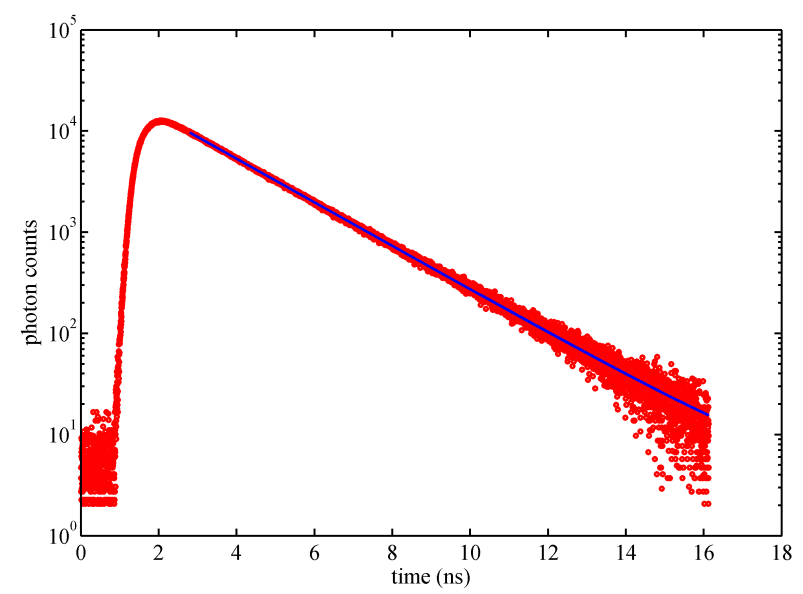

Figure 2.5: Fluorescence lifetime measurement of Atto 655 with exponential fit ( $\tau_{F}=$ 2.01ns)

The electron systems of fluorescent dyes typically include two different types of quantummechanical states. The singlet states with a total electron spin of zero, and the triplet states with a total spin of one (fig. 2.4a). The ground state and first excited state are singlet states. The transition from the excited state to the triplet state involves the flipping of one electron spin, which only occurs with a low probability. More precisely, the transition is forbidden in a perfectly isolated system, due to the conservation of angular momentum. In real systems the transition from singlet to triplet state can occur due to the exchange of angular momentum between the molecule and its surroundings, or the spin-orbit coupling of the electronic states. However, both processes are weak interactions, explaining the low probability of the singlet to triplet transition.

As a consequence, molecules can stay in the triplet state for several microseconds. In the triplet state they are effectively dark, as they cannot fluoresce until they return to the singlet state. Most molecules can be well described by a system of three differential equations (including the first two singlet states and the first triplet state). With the transition rates defined in figure $2.4 \mathrm{a}$, the differential equations are 


$$
\frac{d}{d t}\left[\begin{array}{l}
S_{0}(\mathbf{r}, t) \\
S_{1}(\mathbf{r}, t) \\
T_{1}(\mathbf{r}, t)
\end{array}\right]=\left[\begin{array}{ccc}
-k_{12}(\mathbf{r}) & k_{21} & k_{31} \\
k_{12}(\mathbf{r}) & -\left(k_{23}+k_{21}\right) & 0 \\
0 & k_{23} & -k_{31}
\end{array}\right] \cdot\left[\begin{array}{c}
S_{0}(\mathbf{r}, t) \\
S_{1}(\mathbf{r}, t) \\
T_{1}(\mathbf{r}, t)
\end{array}\right] .
$$

Another import aspect of fluorescent dyes is the spatial distribution of emitted fluorescence light. Most fluorescent dyes behave similar to ideal electrical dipole emitters (like a small rod antenna). Therefore, absorption and detection efficiency for a fixed direction strongly depend on the orientation of the dye molecule. The efficiency for linearly polarized excitation or detection is simply proportional to the square of the scalar product of the electric field vector and the molecular absorption or emission dipole vector:

$$
\kappa(\mathbf{v})=\kappa_{0}(\mathbf{v} \cdot \hat{\mathbf{E}})^{2}
$$

where $\kappa_{0}$ is the efficiency for perfectly aligned dipole, $\mathbf{v}$ is the unit vector along the orientation of the dipole and $\hat{\mathbf{E}}$ the unit vector along the direction of electric field.

For most measurements in solution this is of little consequence, as one averages over all possible orientations. However, this will have a noticeable effect in cases where either free rotation of the dyes is hindered or the time resolution of the measurement is faster than the rotation of the dyes.

\subsubsection{Fluorescence Correlation Spectroscopy}

Fluorescence Correlation Spectroscopy (FCS) is a collective term for several techniques that evaluate the intensity fluctuations of a fluorescence signal to measure the behavior of a molecule or system, typically in aqueous solution. In the last twenty years, rapid development of single photon detectors, optical filters, pulsed diode lasers and single photon counting hardware has led to many improvements and numerous new detection schemes for FCS. Modern FCS systems are typically based on a standard confocal fluorescence microscope (see fig. 2.6). In a confocal microscope, the excitation light is focused into the sample by an objective with high numerical aperture (N.A.). Typical water immersion objectives, with a built-in adjustment for different cover slide thicknesses, allow for an almost ideal focus in aqueous solutions. The fluorescence light is collected through the same objective (epi-fluorescence) and separated from the excitation light by a dichroic mirror. The emitted light is then focused on a small aperture, the confocal pinhole. Emitted light from molecules below or above the focal plane will be blocked by the pinhole. This is the reason for the superior reduction in background fluorescence of the confocal detection scheme compared to the first FCS experiments, described in the introduction. After the pinhole, the light is either imaged directly on a Single Photon Avalanche Diode (SPAD), or split by color or polarization and imaged on several SPADs. Modern detection electronics can record single photon time traces of several such detectors simultaneously. Hence, it is not only possible to calculate the autocorrelation of the fluorescence intensity, but also the cross-correlation between signals at different wavelengths or with different polarization.

The advantage of this confocal detection scheme is the small size of the detection volume, with a lateral diameter of approximately $0.5 \mu \mathrm{m}$ and axial extension of $2 \mu \mathrm{m}$. Due to this small 


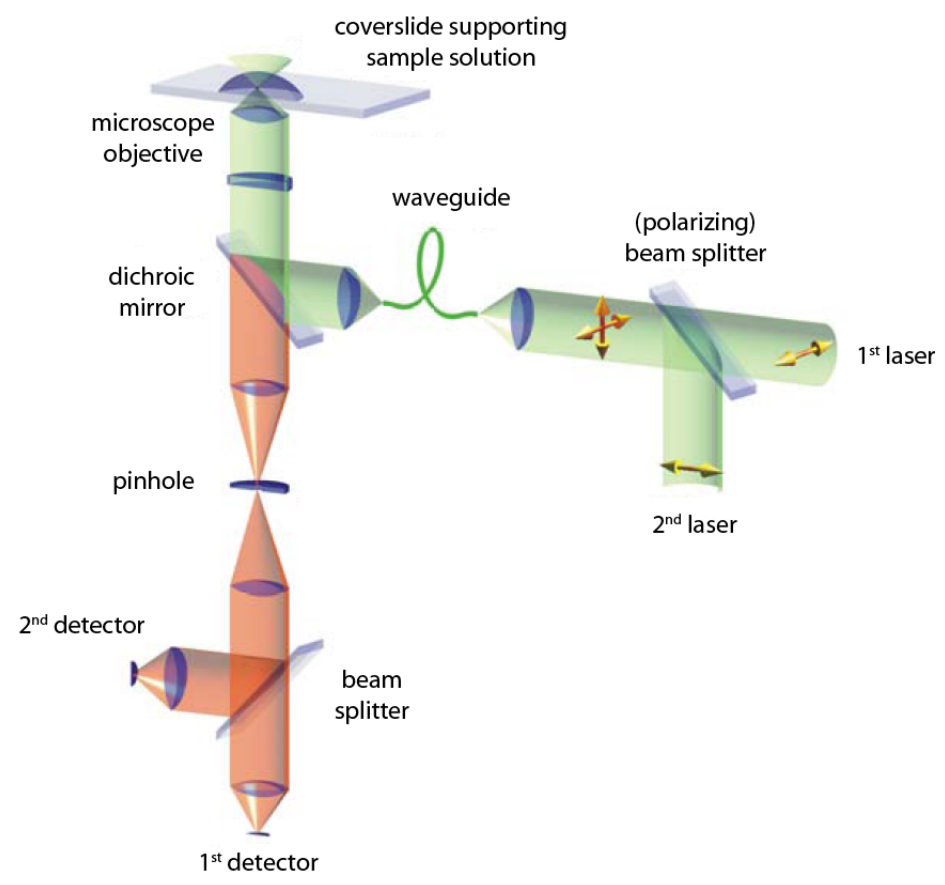

Figure 2.6: Schematic of a confocal FCS setup.

detection volume and the sensitivity of single photon detectors and modern detection electronics, the intensity fluctuations of single fluorecent molecules diffusing through the detection volume can be recorded. Typical sources of fluorescence intensity fluctuations are the diffusing particles in the detection volume, dye photophysics, dye reorientation with respect to the excitation and/or detection polarization, or changes in energy transfer or quenching efficiency. Some of these effects can be suppressed to better seperate contributions from the quantity of interest, for example by selecting a dye with little photophysics or by attaching the molecule to a surface or embedding it in a gel. By choosing an adequate system and setting the right experimental conditions for the measurement, it is possible to measure directly quantities such as diffusion ${ }^{[91,63,40]}$ or photophysics ${ }^{[47,110,112]}$ and also other processes such as binding and reaction kinetics $^{[90,58]}$, conformational dynamics of proteins ${ }^{[29,108]}$ or flow velocities ${ }^{[76,43,6]}$.

The temporal behavior of a system measured in an FCS experiment is obtained by correlating the fluorescence intensity trace $I(t)$ with itself shifted by varying delay times $\tau$, resulting in the Autocorrelation Function (ACF)

$$
g(\tau)=\langle I(t) I(t+\tau)\rangle_{t}
$$

where the brackets denote averaging over all values of $t$. The ACF gives a measure for the probability of detecting a photon at time $t+\tau$ if a photon was detected at time $t$. If photons are emitted from two separated molecules, these events are statistically independent and contribute only in the form of a constant offset to the autocorrelation curve. The time dependent part of an ACF originates solely from contributions of single molecules, and is thus an accurate 
representation of the single molecule dynamics provided that the sample is homogeneous.

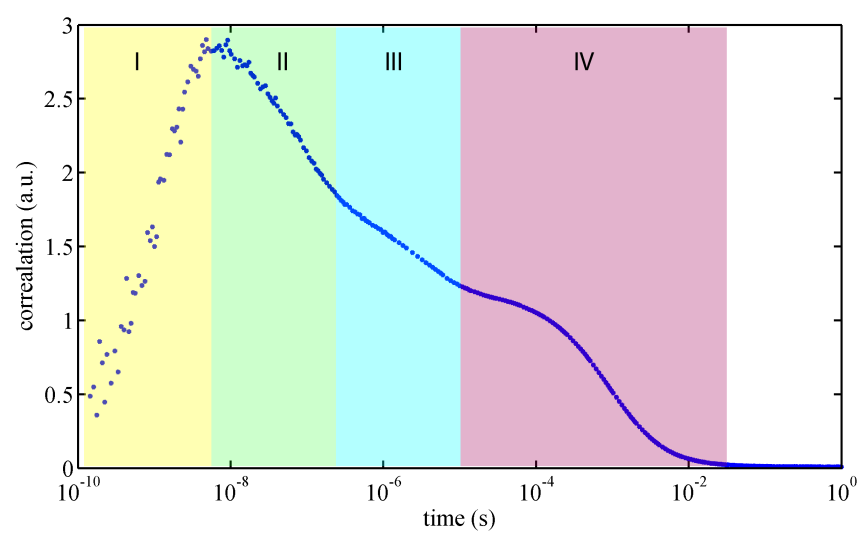

Figure 2.7: Full correlation curve of aldolase labeled with Cy5 with $\tau$ ranging from nanosecond to second delay times. The contributions of different processes to the shape of the curve are clearly separable: Fluorescent antibunching (I), rotational diffusion (II), dye photophysics (III) and translational diffusion (IV)

At very short delay times (few nanoseconds), the ACF is dominated by fluorescence antibunching, a steep rise from the constant offset of the curve (see Fig. 2.7, I). The antibunching dip is due to the fact that each molecule remains in the excited state for a short period of time after excitation. On average this period is as long as the fluorescent lifetime $\tau_{F}$. Hence, the emission of two closely following photons is very unlikely. On the nanosecond to microsecond time range a decay due to rotational diffusion of the molecule might occur (see Fig. 2.7, II). In the microsecond time range any photophyics of the dye, such as triplet state transitions or cistrans isomerization will dominate the ACF (see Fig. 2.7, III). Finally, from the the millisecond time range on, the correlation decays due to the diffusion of the molecule out of the detection volume (see Fig. 2.7, IV).

For some applications of fluorescence correlation spectroscopy, different fluorescence signals are recorded simultaneously. This is done, if one can extract additional information about the system of interest from the different fluorescence signals (e.g. different wavelengths or polarization). In these cases one can gain additional information from the correlation between the different signals represented by the Cross-Correlation Function (CCF)

$$
g_{a b}(\tau)=\left\langle I_{a}(t) I_{b}(t+\tau)\right\rangle_{t}
$$

where $I_{a}$ and $I_{b}$ are now the intensity traces measured in two different detection channels. The time dependent part of the CCF will only have a non vanishing amplitude if the two signals are correlated. This would only be the case if e.g. two different dyes are interacting and diffusing together at the same time through the detection volume, leading to a higher probability of detecting a photon in the one channel shortly after a photon was detected in the other channel. The temporal behavior of the $\mathrm{CCF}$ in such experiments will therefore mostly depend on diffusion, whereas the amplitude of the CCF contains information about the degree of interaction of the two dye molecules. 


\subsubsection{The Correlation Function}

The exact shape of the diffusion part (see Fig. 2.7) of the ACF and CCF is determined by the shape of the detection volume or more accurately the Molecular Detection Function (MDF). The MDF contains the spatial dependence of the excitation intensity and the detection efficiency. In the most general case, the MDF also contains any dependence of the excitation (detection) probability on dye orientation and excitation intensity ${ }^{[33]}$. Because of its simplicity and the ease of following calculations the most commonly used model for the MDF of a confocal system is a three dimensional Gaussian:

$$
U(\mathbf{r})=e^{-2\left(x^{2}+y^{2}\right) / w_{x y}^{2}} e^{-2 z^{2} / w_{z}^{2}}
$$

where the z-coordinate is along the optical axis, $w_{x y}$ is the extension of the MDF in the radial direction and $w_{z}$ is the extension along the optical axis. A more accurate model will be introduced in the next section in the context of dual focus FCS. Although the parameters $w_{x y}$ and $w_{z}$ can be calculated if the parameters of the optical system are exactly known, they are usually determined by calibration measurements.

To calculate the exact form of the ACF on can use the solution of the diffusion equation obtained earlier (eq. 2.4). The contribution of diffusion to the ACF can be written as the probability of detecting a molecule at position $\mathbf{r}_{0}$ multiplied with the probability of the molecule moving to a position $\mathbf{r}_{1}$ within time $\tau$ and the probability of detecting it at the new location integrated over all possible initial and final positions. The resulting function

$$
g(\tau)=g(\infty)+\epsilon^{2} c \int d \mathbf{r}_{0} \int d \mathbf{r}_{1} U\left(\mathbf{r}_{1}\right) \frac{1}{(4 \pi D \tau)^{3 / 2}} \exp \left[-\frac{\left(\mathbf{r}_{1}-\mathbf{r}_{0}\right)^{2}}{4 D \tau}\right] U\left(\mathbf{r}_{0}\right),
$$

where $c$ is the concentration of molecules and $\epsilon$ is a constant containing the overall excitation intensity, detection efficiency, brightness of a single molecule, etc. For the simple three dimensional Gaussian model of the MDF the integrals in eq. 2.33 evaluate to:

$$
g(\tau)=g(\infty)+A\left(1+\frac{\tau}{\tau_{D}}\right)^{-1}\left(1+a^{2} \frac{\tau}{\tau_{D}}\right)^{-1 / 2}
$$

where $a=\omega_{x y} / \omega_{z}$ is the ratio of axial and radial extension of the MDF, and the diffusion time $\tau_{D}$ is given by

$$
\tau_{D}=\frac{\omega_{x y}^{2}}{4 D}
$$

The amplitude $A$ of the time dependent part is given by

$$
A=\frac{1}{8} \epsilon^{2} c \pi^{3 / 2} \omega_{x y}^{2} \omega_{z} .
$$




\subsubsection{Dye Photophysics}

Fluorescent molecules in the triplet state are effectively dark, since they will not fluoresce until they return to the singlet state. As a consequence, the dynamics of the triplet state can be observed as a separate contribution to the time dependent part of the autocorrelation function (Fig. 2.7). This contribution can be derived from the system of differential equations describing the probabilities of the molecule occupying the first two singlet states and the triplet state (eq. 2.28).

Right after the emission of a photon $(t=0)$, the system will be in the initial state

$$
\left[\begin{array}{l}
S_{0}(\mathbf{r}, 0) \\
S_{1}(\mathbf{r}, 0) \\
T_{1}(\mathbf{r}, 0)
\end{array}\right]=\left[\begin{array}{l}
1 \\
0 \\
0
\end{array}\right]
$$

Since the transition from the excited state to the ground state is much faster than the transitions between singlet and triplet states $\left(k_{21} \gg k_{23}, k_{31}\right)$, the time dependent contribution to the probability of occupying the excited state can be approximated as ${ }^{[111]}$ :

$$
S_{1}(\mathbf{r}, t)=(1-T)+T e^{-t / \tau_{T}}
$$

where

$$
T=\frac{k_{12}^{2}(\mathbf{r}) k_{23}}{\left(k_{12}(\mathbf{r})+k_{21}\right)\left[k_{12}(\mathbf{r})\left(k_{23}+k_{31}\right)+k_{21} k_{31}\right]}
$$

and

$$
\tau_{T}=\left[k_{31}+\frac{k_{12}(\mathbf{r}) k_{23}}{k_{12}(\mathbf{r})+k_{21}}\right]^{-1} .
$$

The value $T$ is the fraction of molecules in the triplet state at steady-state conditions, and the time constant $\tau_{T}$ is the characteristic time of the triplet state dynamics. With these results, the autocorrelation function of a system with diffusion and triplet state dynamics can be written as:

$$
g(\tau)=g(\infty)+A\left[1+\frac{T}{1-T} e^{-t / \tau_{T}}\right]\left(1+\frac{\tau}{\tau_{D}}\right)^{-1}\left(1+a^{2} \frac{\tau}{\tau_{D}}\right)^{-1 / 2}
$$

\subsubsection{Rotational Diffusion and Nanosecond timescale FCS}

The process which influences the fluorescence intensity fluctuations most strongly on the nanosecond time range is the rotational diffusion of the fluorescent molecules. The rotation of molecules plays an important role in chemical and enzymatic reactions. Reaction partners do not only have to colocalize, but also have to find the correct orientation to react or bind to each other. In an aqueous solution, the rotation of small molecules is so fast that it will rarely be the limiting step of any reaction. However, it may have a greater impact on reactions 
involving macromolecules or large enzymatic complexes, especially in a viscous or crowded environment as inside a cell.

The standard method for measuring the rotational diffusion of molecules is fluorescence anisotropy ${ }^{[106,107,103,115,67]}$. Here the sample is illuminated with a pulse of linearly polarized light. The anisotropy of the fluorescence emission is then recorded over time. The source of the fluorescence anisotropy is the dipolar character of fluorescence absorption and emission. Most fluorescent dyes behave as electric dipoles in absorption and emission, with a higher probability of absorbing or emitting a photon with the electrical field vector parallel to the dipole axis of the molecule. If a sample is excited with a pulse of linearly polarized light, molecules that become excited are predominantly aligned along the direction of the excitation polarization. The detectable instantaneous fluorescence emission will therefore be strongly polarized along the same direction and will be weaker for the orthogonal polarization, giving rise to fluorescence anisotropy. As the fluorescent molecules undergo rotational diffusion, the orientation of the excited molecules will be randomized and the fluorescence anisotropy will decay (Fig. 2.8).
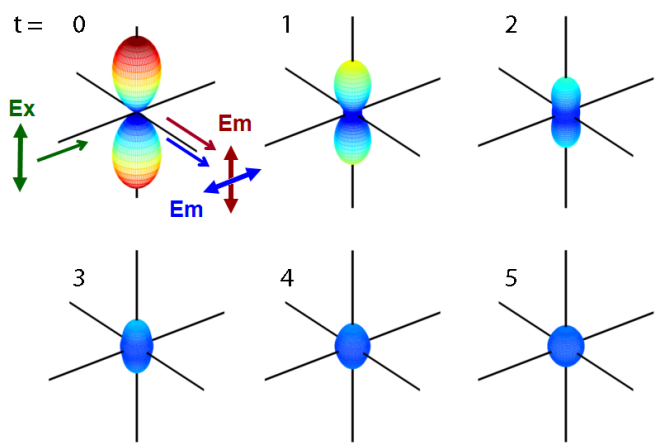

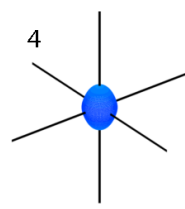

(a)

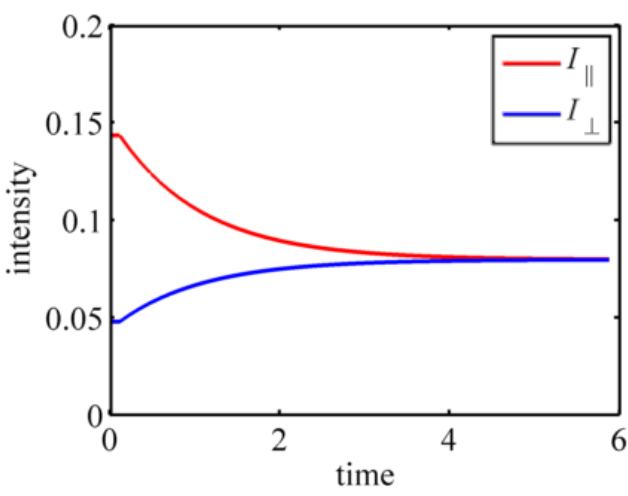

(b)

Figure 2.8: (a): Probability density of finding an excited molecule with a certain dipole orientation after an excitation pulse with linear polarized light (green), assuming uniform distribution of orientations in the sample prior to excitation. The probability for each direction is given by the distance of the surface from the center in this direction. (b): The detected fluorescence in an ideal anisotropy measurement for parallel polarization (red) and orthogonal polarization (blue), as a function of time after the excitation pulse, assuming infinite fluorescence lifetime.

The intrinsic limitation of fluorescence anisotropy is the short time range of accessible rotation times. The fluorescence anisotropy can only be measured for time scales equal to or shorter than the fluorescence lifetime of the used fluorophore. If the molecules rotate much slower than their intrinsic fluorescence decay time, no change in the anisotropy can be observed. One solution to this problem is the use of phosphorescent probes with long phosphorescence decay times ${ }^{[72]}$. The long lifetime of phosphorescent labels can significantly extend 
the range of the anisotropy measurement. However, long luminescence lifetimes mean low emission intensities, making this method less practicable.

An easier and more sensitive alternative is the use of FCS for rotational diffusion measurements. The advantage of this is that standard fluorescence labels with short lifetimes can be used.

The use of FCS for measuring the rotation of molecules had already been suggested in early publications ${ }^{[30,5]}$. In this case, the intensity fluctuations of the fluoresence, emitted with a specific polarization, are analyzed. The intensity fluctuates due to the rotational diffusion of the fluorescence molecules. The first experimental realizations of this technique used linearly polarized Continuous Wave $(\mathrm{CW})$ laser excitation and two separate photo detectors ${ }^{[56,57]}$. By this, the autocorrelation and the cross-correlation of the two polarization directions could be measured in two consecutive experiments. The autocorrelation functions, measured for one specific polarization, show a decay very similar to that of the fluorescence anisotropy measurement (Fig. 2.9). The fast rise in the correlation function at zero delay time is due to fluorescence anti-bunching ${ }^{[2]}$.
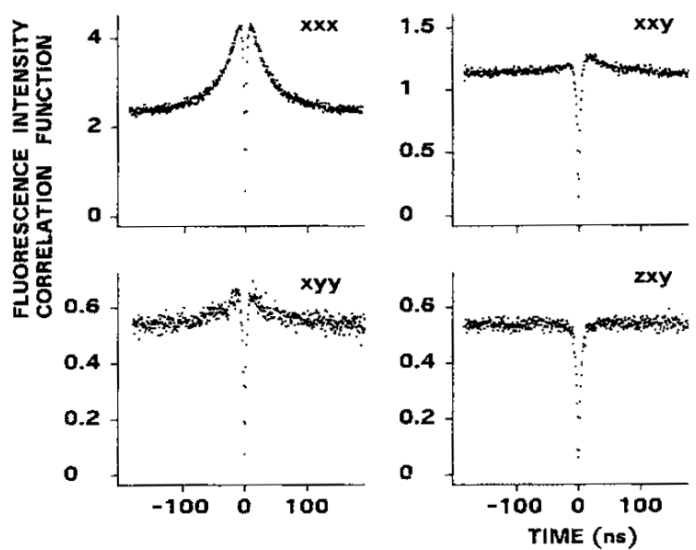

Figure 2.9: Experimental fluorescence intensity correlations as measured by Kask et al. (from ref. [57]). The three letters in each inset denote the axis of orientation for excitation polarization, first detector polarization and second detector polarization respectively (eg. $\mathrm{xxx}$ stands for excitation and both detection polarizations aligned parallel to the $\mathrm{x}$-axis).

The slower decay of the correlation functions is due to the rotation of the dye. If a photon is detected with one specific polarization, the probability of the fluorescent dye being aligned in that direction is high. Consequently, the probability of detecting a second photon with the same polarization directly afterwards is increased since the molecule is already aligned in that direction. This correlation decays as the rotational diffusion randomizes the alignment of the molecule (Fig. 2.8a). Since the correlation uses two subsequent photons to probe the reorientation of the molecule, there is no upper limit for the measurement time range, in contrast to fluorescence anisotropy. However, a rotation faster than the fluorescence lifetime of the dye can not be measured, as there will rarely be two photons from the same molecule within such a short time. The time range of FCS in measuring rotational diffusion is therefore complementary to that of fluorescence anisotropy. Altogether, FCS is a very suitable method for the 
measurement of large macromolecules and of rotation in viscous solutions.

Other techniques for measuring the rotational diffusion of macromolecules include Nuclear Magnetic Resonance (NMR) ${ }^{[65]}$ and Electron Paramagnetic Resonance (EPR ${ }^{[14]}$. However, these methods need special sample preparation and measurement conditions such as deuterated water, and hence are not applicable to many systems. The high sample concentrations required to use these techniques might lead to aggregation of many biomolecules. Fluorescence based techniques use nanomolar sample concentration and can be used in any solution or environment that is optically transparent. Thus, FCS on the nanosecond time-scale should be the method of choice for observing the rotational diffusion of molecules in a cell-like environment.

In summary, there are several cases that have to be distinguished when measuring the rotational diffusion of a molecule with fluorescence-based methods:

1. $\tau_{F} \gg \tau_{D}$ : for molecules that rotate much faster than the fluorescent lifetime of the dye any information on the orientation is lost and the rotation can not be measured in a fluorescence experiment.

2. $\tau_{F} \cong \tau_{D}$ : for molecules that rotate approximately as fast as the fluorescent lifetime the rotation can be determined with a fluorescence anisotropy measurement ${ }^{[107,67]}$.

3. $\tau_{F} \ll \tau_{D}$ : for molecules with a rotation time much longer than the fluorescent lifetime the rotation can best be measured by an FCS experiment.

The latter applies to large macromolecules with rotation times larger than the fluorescence lifetime of typical dyes. In this case it is only possible to measure molecular rotation with FCS. The correlation function for the fluorescence intensity from such a system can be written in a way very similar to that for translational diffusion, eq. 2.33. Using Green's function of the rotational diffusion equation from equation 2.25 , one obtains

$$
\begin{array}{r}
\left\langle\kappa_{a}^{\alpha}\left(t_{0}\right) \kappa_{b}^{\beta}\left(t_{0}+\tau\right)\right\rangle_{t_{0}}=\int d \mathbf{r} \int d \omega \int d \Omega \int d \Omega^{\prime} U_{a}^{\alpha}[R(\Omega) \omega, \mathbf{r}] \\
\times G\left(\Omega, \Omega^{\prime}, \tau\right) U_{b}^{\beta}\left[R\left(\Omega^{\prime}\right) \omega, \mathbf{r}\right] \\
=\int d \mathbf{r} \int d \omega \int d \Omega \int d \Omega^{\prime}\left[R^{-1}(\Omega) U_{a}^{\alpha}(\omega, \mathbf{r})\right] \\
\times G\left(\Omega, \Omega^{\prime}, \tau\right)\left[R^{-1}\left(\Omega^{\prime}\right) U_{b}^{\beta}(\omega, \mathbf{r})\right]
\end{array}
$$

where $U_{a}^{\alpha}[\omega, \mathbf{r}]$ is the position and orientation dependent probability of detecting a photon for excitation with polarization $a$ and detection with polarization $\alpha$. The integration extends over all possible orientations of the absorption/emission dipole with respect to the molecular reference frame $\omega$, all possible positions $\mathbf{r}$ and all possible initial and final molecular orientations $\Omega$ and $\Omega^{\prime}$.

The orientation dependence enters the detection probability in the form described in eq. 2.29 and the position dependence is that of the excitation intensity distribution. For the idealized case of negligible optical aberrations and plane wave excitation, this probability is 


$$
U_{a}^{\alpha}(\omega, \mathbf{r})=\left(\mathbf{v} \cdot \hat{\mathbf{x}}_{\mathbf{a}}\right)^{2}\left(\mathbf{v} \cdot \hat{\mathbf{x}}_{\alpha}\right)^{2} u_{a}(\mathbf{r}) u^{\alpha}(\mathbf{r})
$$

where $u_{a}(\mathbf{r})$ is a function containing the position-dependent electric field distribution as generated by the focused excitation laser, and $u^{\alpha}(\mathbf{r})$ inlcudes the position dependent detection efficiency of the confocal detection. Both functions have to be calculated for the case that the principal axes of the molecule are parallel to the axes of the lab frame.

The $U_{a}^{\alpha}$ can be expanded into a series of Wigner matrices, since they form a complete orthogonal function set, in the form

$$
U_{a}^{\alpha}(\omega, \mathbf{r})=\sum_{l=0}^{\infty} \sum_{k, m=-l}^{l} \sqrt{\frac{2 l+1}{8 \pi^{2}}}\left(u_{a}^{\alpha}\right)_{k m}^{l}(\mathbf{r}) D_{k m}^{l}(\phi, \theta, \psi)
$$

where $\left(u_{a}^{\alpha}\right)_{k m}^{l}(\mathbf{r})$ are the position dependent expansion coefficients. The $U_{a}^{\alpha}(\omega, \mathbf{r})$ are fourth order polynomials of the components of the absorption and emission dipole vectors, and the maximum value of $l$ yielding non-zero coefficients is therefore four. Using this expansion and inserting Green's function into equation 2.42 gives the compact result

$$
\left\langle\kappa_{a}^{\alpha}\left(t_{0}\right) \kappa_{b}^{\beta}\left(t_{0}+\tau\right)\right\rangle_{t_{0}}=\sum_{l=0}^{4} \sum_{m=-l}^{l}\left(f_{a b}^{\alpha \beta}\right)_{m}^{l} e^{-\left[D_{\perp} l(l+1)+\left(D_{\|}-D_{\perp}\right) m^{2}\right] \tau} .
$$

The coefficients $\left(f_{a b}^{\alpha \beta}\right)_{m}^{l}$ can be calculated analytically for the idealized case of plane wave excitation as has been done in [86]. One important modification made here, in contrast to the first rotation measurements with FCS, is the inclusion of the possibility of different excitation polarizations during the same experiment. With modern pulsed laser sources it is possible to determine correlation curves for many different polarization combinations in a single experiment. In the measurement scheme used in this work, two orthogonally polarized lasers are pulsed alternatingly to determine correlation curves for all possible polarization combinations simultaneously. The measurement scheme will be described in more detail later and is identical to the one used in reference [86].

\subsubsection{Dual Focus FCS}

One of the most widespread applications for fluorescence correlation spectroscopy is the determination of diffusion coefficients in solution. Exact knowledge of the diffusion coefficient allows to calculate the size of a molecule from the Stokes-Einstein relation (eq. 2.12). To calculate an accurate diffusion coefficient one needs to know the exact size of the detection volume. The larger the detection volume the longer it takes on average for a molecule to diffuse through the volume. This leads to a slower decay of the fluorescence intensity correlation. As a consequence, the diffusion coefficient will be underestimated if a smaller volume than the actual volume is assumed, and vice versa.

For many cases, the volume can be determined with a simple calibration measurement using a reference substance with known diffusion coefficient. An alternative to this method is to record the confocal image of a small fluorescent sphere. Typically, the image of small sphere attached to a cover slide is recorded by scanning the sample with a piezo electric sample stage. 
The resulting three dimensional image of the sphere will be identical to the detection efficiency distribution and can be used to calculate the detection volume ${ }^{[93]}$.

Modern microscope objectives are well corrected for chromatic and spherical aberrations as well as cover slide thickness. However, these corrections work only for one specific refractive index of the sample and one specific cover slide thickness. There will be variations in the exact shape of the detection volume if either of the two parameters differ. Surprisingly, small deviations can already lead to significant errors of the shape and size of the detection volume $^{[34,33]}$. Small variations in cover slide thickness, even from the same batch, can already lead to measurement errors. These errors can only be excluded when using the same cover slide for reference and actual measurement. Additionally, any remaining chromatic aberrations will lead to different detection volume sizes and focal positions for different wavelengths. Therefore, the detection volume measured with a reference sample might be different from the one in the actual measurement if the emission spectra of the dyes differ.

In aqueous solutions, if refractive index and cover slide thickness are well known the aforementioned effects are in some cases neglectable if proper care is taken. In other cases, like measurements inside cells, an accurate calibration measurement might not be possible. Here the inhomogeneous refractive index of the sample can lead to large measurement errors. Dual Focus Fluorescence Correlation Spectroscopy (2fFCS) solves this problem by introducing an external length scale which is robust with respect to changes in the refractive index.

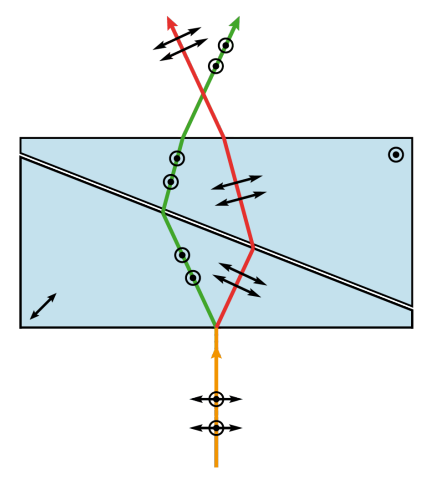

(a)

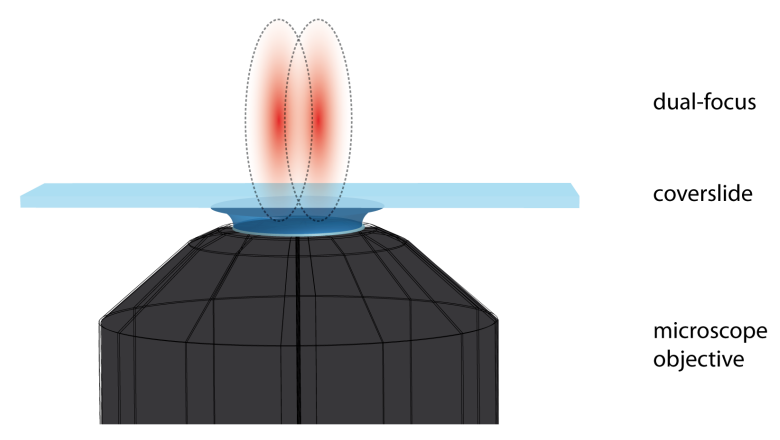

(b)

Figure 2.10: (a): Schematic of Nomarski prism, showing a beam with in-plane polarization (red) and out-of-plane polarization (green). (b): Schematic of the two foci generated in the sample solution of a dual focus FCS setup.

In 2fFCS, two foci are generated by introducing a Nomarski prism (Fig. 2.10a) in the back focal plane of the microscope's objective. The light of a laser beam passing through the prism is deflected into a different direction depending on the polarization of the beam. The different 
deflection angle of two orthogonally polarized laser beams results in the generation of two spatially separated but overlapping laser foci in the focal plane of the objective (see Fig. 2.10b). The distance of the two foci in 2fFCS is only dependent on the deflection angle introduced by the Nomarski prism and is, in contrast to the previously mentioned measurement schemes, insensitive to optical aberrations and especially to refractive index changes.

With dual-focus FCS, the Cross-Correlation Function (CCF) between the signals from the two excitation volumes can be calculated, in addition to the Autocorrelation Functions (ACFs) of each focus. The temporal behavior of the diffusion part of the CCF is dominated by the time a molecule needs to diffuse from one focal volume to the other, thus it is only dependent on the distance between the excitation volumes but not their exact shape. This makes $2 \mathrm{fFCS}$ robust against variations in MDF shape, which in many cases limits the accuracy of conventional FCS.

For an alignment of the two excitation volumes along the $x$-axis, the CCF for a diffusing molecule is analogous to eq. 2.33

$$
g_{\delta}(\tau)=g_{\delta}(\infty)+\epsilon_{0} \epsilon_{1} c \int d \mathbf{r}_{0} \int d \mathbf{r}_{1} U\left(\mathbf{r}_{1}\right) \frac{1}{(4 \pi D \tau)^{3 / 2}} \exp \left[-\frac{\left(\mathbf{r}_{1}-\mathbf{r}_{0}-\hat{\mathbf{x}} \delta\right)^{2}}{4 D \tau}\right] U\left(\mathbf{r}_{0}\right)
$$

where $\epsilon_{0}$ and $\epsilon_{1}$ are now describing the excitation intensities and molecular brightness in their respective excitation volume, $\delta$ is the center distance between the two excitation volumes, and $\hat{\mathbf{x}}$ the unit vector along the $\mathrm{x}$-axis.

From now on, a more realistic model for the MDF will be used as it allows for better fit quality and hence improved accuracy. A good choice is the model proposed by Dertinger et $a l .{ }^{[24]}$ where

$$
U(\mathbf{r})=\frac{\kappa(z)}{w^{2}(z)} \exp \left[-\frac{2}{w^{2}(z)}\left(x^{2}+y^{2}\right)\right]
$$

In each plane perpendicular to the optical axis, the MDF is again approximated by a Gaussian distribution, but the shape along the optical axis is described by a hyperbolic profile

$$
w(z)=w_{0}\left[1+\left(\frac{\lambda_{e x} z^{2}}{\pi w_{0}^{2} n}\right)\right]^{1 / 2}
$$

with the amplitude function

$$
\kappa(z)=2 \int_{0}^{a} d \rho \frac{\rho}{R^{2}(z)} \exp \left(-\frac{2 \rho^{2}}{R^{2}(z)}\right)=1-\exp \left(-\frac{2 a^{2}}{R^{2}(z)}\right)
$$

where $R(z)$ is given by

$$
R(z)=R_{0}\left[1+\left(\frac{\lambda_{e m} z^{2}}{\pi R_{0}^{2} n}\right)\right]^{1 / 2}
$$


The parameters are the excitation wavelength $\lambda_{e x}$, the mean emission wavelength $\lambda_{e m}$, the refractive index of the medium $n$, and the radius of the confocal aperture divided by magnification $a$. The two free parameters $w_{0}$ and $R_{0}$ are not known a priori and have to be determined experimentally.

Using this more accurate MDF (eq. 2.47), and changing variables to

$$
\xi=\frac{z_{1}-z_{0}}{2 \sqrt{D \tau}}, \eta=\frac{z_{1}+z_{0}}{2}
$$

one obtains for the $\mathrm{CCF}$

$$
\begin{aligned}
g_{\delta}(\tau)=g_{\delta}(\infty)+\frac{\epsilon_{0} \epsilon_{1} c \sqrt{\pi}}{2} \int_{0}^{\infty} & d \xi \int_{0}^{\infty} d \eta \frac{\kappa(\eta-\sqrt{D \tau} \xi) \kappa(\eta+\sqrt{D \tau} \xi)}{8 D \tau+w^{2}(\eta-\sqrt{D \tau} \xi)+w^{2}(\eta+\sqrt{D \tau} \xi)} \times \\
& \exp \left[-\xi^{2}-\frac{2 \delta^{2}}{8 D \tau+w^{2}(\eta-\sqrt{D \tau} \xi)+w^{2}(\eta+\sqrt{D \tau} \xi)}\right]
\end{aligned}
$$

Integration over $\xi$ and $\eta$ can be done numerically over an area with $\mid \eta \pm \sqrt{D \tau} \xi) \mid<M$ where $M$ is chosen in such a way that increasing $M$ does not further change the integration result.

\subsubsection{Measuring Flow Velocities with FCS}

Although it is possible to measure the flow velocities in solution with conventional $\operatorname{FCS}^{[76,43]}$ these measurements are hampered by the same problems that make accurate diffusion measurements so difficult. Good knowledge of the MDF is necessary, but even then it is not possible to determine the direction of the flow.

$2 \mathrm{fFCS}$, on the other hand, is an excellent tool for measuring flow velocities because the two excitation volumes do not only allow for extremely accurate measurements along the axis of the focal displacement but also for determining the direction of the flow. The CCF (eq. 2.46) changes only slightly in the presence of flow. Then, one obtains

$$
g_{\delta, \mathbf{v}}(\tau)=g_{\delta, \mathbf{v}}(\infty)+\epsilon_{0} \epsilon_{1} c \int d \mathbf{r}_{0} \int d \mathbf{r}_{1} U\left(\mathbf{r}_{1}\right) \frac{1}{(4 \pi D \tau)^{3 / 2}} \exp \left[-\frac{\left(\mathbf{r}_{1}-\mathbf{r}_{0}-\hat{\mathbf{x}} \delta-\mathbf{v} \tau\right)^{2}}{4 D \tau}\right] U\left(\mathbf{r}_{0}\right)
$$

where $\mathbf{v}$ is the flow vector. Using the same MDF as before and changing variables according to 2.51 , the $\mathrm{CCF}$ is

$$
\begin{aligned}
g_{\delta, \mathbf{v}}(\tau)=g_{\delta, \mathbf{v}}(\infty)+ & \frac{\epsilon_{0} \epsilon_{1} c \sqrt{\pi}}{2} \int_{0}^{\infty} d \xi \int_{0}^{\infty} d \eta \frac{\kappa(\eta-\sqrt{D \tau} \xi) \kappa(\eta+\sqrt{D \tau} \xi)}{8 D \tau+w^{2}(\eta-\sqrt{D \tau} \xi)+w^{2}(\eta+\sqrt{D \tau} \xi)} \times \\
& \exp \left[-\left(\xi-\frac{v_{z}}{2} \sqrt{\frac{\tau}{D}}\right)^{2}-\frac{2\left(\delta-v_{x} \tau\right)^{2}+2\left(v_{y} \tau\right)^{2}}{8 D \tau+w^{2}(\eta-\sqrt{D \tau} \xi)+w^{2}(\eta+\sqrt{D \tau} \xi)}\right]
\end{aligned}
$$

Integration over $\xi$ and $\eta$ is done numerically as for the $2 \mathrm{fFCS}$ measurements without flow. 


\section{Materials and Methods}

\subsection{Sample Preparation}

Most molecules and proteins do not strongly fluoresce in the visible light spectrum and therefore need to be coupled to a small fluorescent marker molecule to be studied with FCS. If one wants to measure the rotation of the molecule, one has to assure that the fluorescent marker co-rotates with the molecule.

For fluorescence microscopy and many other techniques, it has become standard practice to create fusion constructs of a protein of interest with a fluorescent protein such as Green Fluorescent Protein $(\mathrm{GFP})^{[15]}$. However, fluorescent proteins are quite large and will therefore significantly change the hydrodynamic radius, diffusion coefficient, and potentially other properties of a molecule. Small organic dyes such as rhodamine or cyanine derivatives are sufficiently small to have a negligible effect if bound to large proteins or macromolecules. Most fluorescent dyes are available with special reactive end groups that readily react with common functional groups on proteins such as amino groups or sulfohydryl groups.

\subsubsection{Labeling of Proteins}

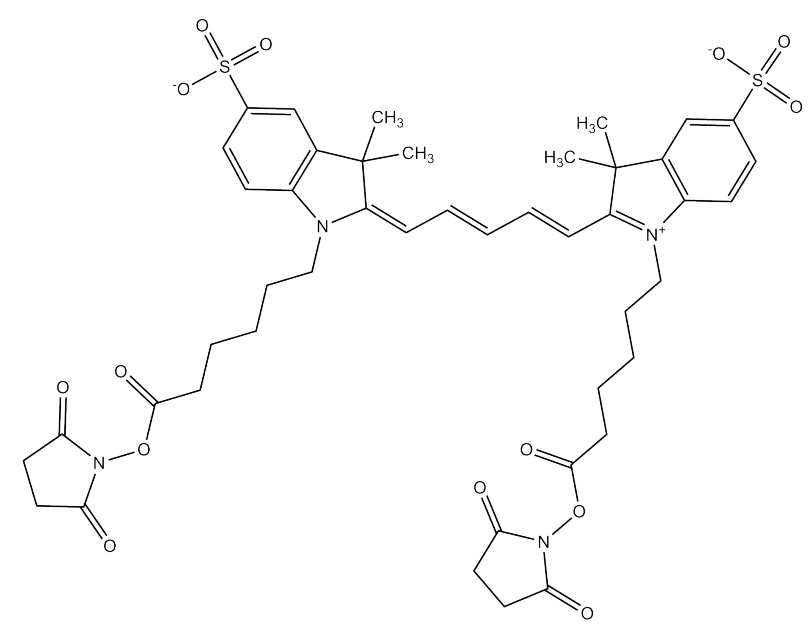

Figure 3.1: Structure of bis-functional dye Cy5

For FCS measurements in crowded solutions, aldolase (gel filtration calibration kit HMW, GE Healthcare) was labeled with Cy5 bis-succimedyl ester (GE Healthcare Europe GmbH, Freiburg, Germany) (Fig. 3.1). For labeling, aldolase was dissolved at a concentration of approximately $10 \mathrm{mg} / \mathrm{mL}$ in a $50 \mathrm{mM}$ carbonate buffer with $\mathrm{pH} 8.4$. After addition of Cy5 
dissolved in Dimethyl Sulfoxide (DMSO), the reaction was incubated at room temperature in the dark for two hours. Afterwards, free dye was removed from labelled aldolase by High-Performance Liquid Chromatography (2000 series HPLC, Jasco Labor und Datentechnik GmbH, Groß-Umstadt, Germany) using a PROTEEMA protein purification column (PSS Polymer Standards Service GmbH, Mainz, Germany). Labeled proteins were stored at $-80^{\circ} \mathrm{C}$ before use.

\subsection{FCS Setup}

All FCS measurements were done on a commercial confocal microscopy system for time resolved measurements (MicroTime 200 with dual-focus option, PicoQuant GmbH, Berlin, Germany). The main components of the system are a standard inverted microscope (IX71, Olympus Deutschland GmbH, Hamburg, Germany) with water immersion objective (UPLSAPO 60XW, Olympus Deutschland GmbH, Hamburg, Germany), four SPADs (two SPCM-AQR13, PerkinElmer Optoelectronics, Wiesbaden, Germany, and two MPDs, Bolzano, Italy), and a single-photon counting electronics (HydraHarp 400, PicoQuant GmbH, Berlin, Germany).

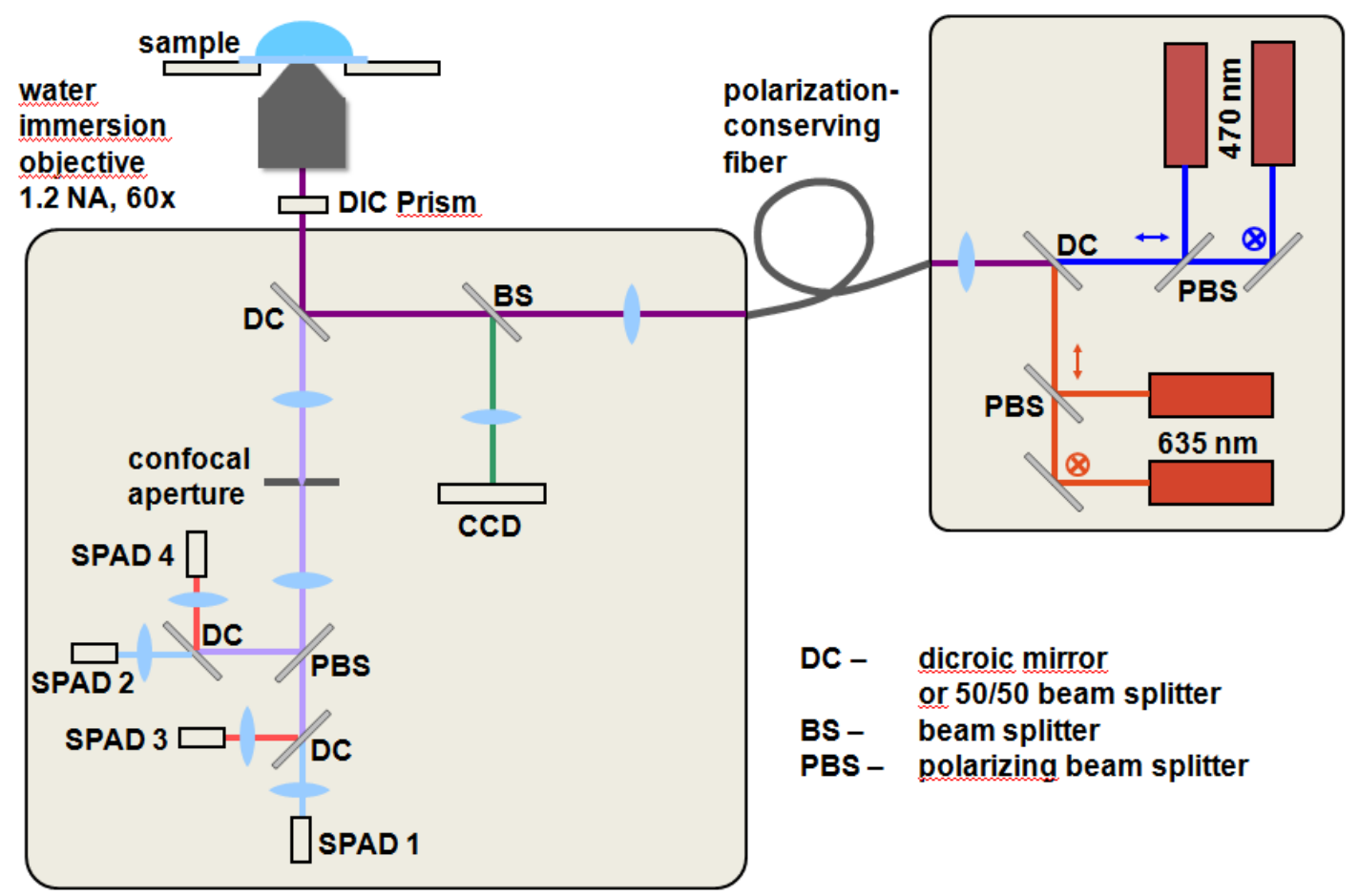

Figure 3.2: Schematic of the setup used for FCS measurements.

Two identical pulsed diode lasers with $640 \mathrm{~nm}$ wavelength are pulsed alternatingly by a computer-controlled diode laser driver (PDL 828, PicoQuant GmbH, Berlin, Germany). The lasers generate orthogonally polarized beams that are combined with a polarizing beam splitter 
and coupled into a polarization preserving optical fiber (Fig. 3.2). For 2fFCS measurements, the lasers are pulsed with a repetition rate of $20 \mathrm{MHz}$ and an interpulse distance of $25 \mathrm{~ns}$. For measurements of rotational diffusion a repetition rate of $80 \mathrm{MHz}$ and a pulse distance of $6.25 \mathrm{~ns}$ is used. For rotational measurements, the time delay is generated by inserting a cable of appropriate length between the pulse generator and the laser driver. Typical average laser powers were $10 \mu \mathrm{W}$ per laser for $2 \mathrm{fFCS}$ and $200 \mu \mathrm{W}$ per laser for rotation measurements.

At the output of the fiber, the light is collimated by a microscope objective (UPLSAPO 10x, Olympus) and reflected into the microscope by a dichroic mirror. For the 2fFCS measurement, the two polarizations are split by a Nomarski prism (U-DICTHC, Olympus) into two slightly tilted beams that are focused into two slightly overlapping spots in the focal plane of the objective. Fluorescence is collected through the same objective and passed through the dichroic mirror (51008 bs, Chroma Technology Corporation, Bellows Falls, USA). The emitted light is focused on a pinhole which serves as a confocal aperture. The size of the pinhole is $150 \mu \mathrm{m}$ which is larger than typically used for confocal imaging but necessary to let the light from the two shifted foci pass at the same time.

Afterwards, the light is collimated again and split by a polarizing beam splitter followed by two 50/50 beam splitters. Each beam is focused on a separate SPAD, which are all connected to a photon-counting hardware. Splitting of the emission light onto four detectors is necessary for the rotation measurements, so that the CCFs for all possible polarization combinations can calculated, despite the finite dead time of each detector. For the 2fFCS measurements, the $50 / 50$ beam splitters can be removed and fluorescence is recorded with only two detectors. The detected photons can be assigned to their corresponding excitation pulse by their exact arrival time in relation to the pulse sequence, yielding two photon traces for each detector (Fig. 3.3).

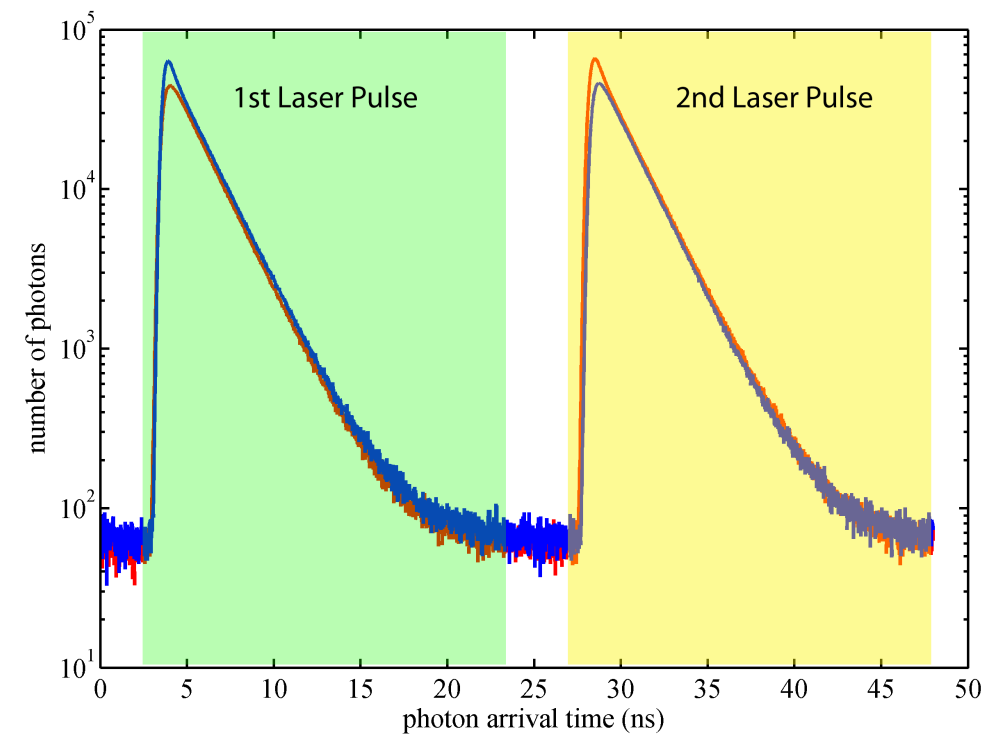

Figure 3.3: Photon arrival time histogram with time gates for the first laser pulse (green) and second laser pulse (yellow) 


\subsubsection{Flow Measurements in Microfluidic Devices}

Flow velocity measurements were performed in microfluidic chips produced from a polydimethylsiloxane (PDMS) mold attached to a cover slide (Gerhard Menzel GmbH, Braunschweig, Germany). Laminar flow of the sample solution was generated using a syringe pump (Nexus 3000, Chemyx) with a gas-tight syringe (1700 Series, Hamilton). The syringe was connected to the microfluidic chip with a short piece (approx. $10 \mathrm{~cm}$ ) of PTFE tubing.

Flow velocity measurements were performed with the hydrophobic dye ATTO Oxa12 (ATTOTEC GmbH, Siegen, Germany) dissolved at nano molar concentrations in liquid crystal 4Pentyl-4'-Cyanobiphenyl (5CB) (Fig. 3.4). For control measurements, the hydrophilic dye ATTO 655 dissolved in a mixture of $73 \mathrm{wt} \%$ glycerol and water was used.

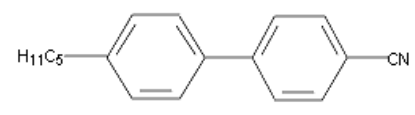

Figure 3.4: Structure of the liquid crystal 4-Pentyl-4'-cyanobiphenyl (5CB)

For each flow profile, a set of point measurements was performed for points on a line perpendicular to the flow direction and parallel to the cover slide. Point measurements were automatically performed with a piezo nano-positioning stage (P-562.3CD, Physik Instrumente, Karlsruhe, Germany). Measurement time at each point was two minutes.

\subsection{Data Analysis}

\subsubsection{Calculation of Correlation Curves}

Calculation of correlation curves from single-photon time traces is done using custom Matlab routines similar to the ones described in [102]. Logarithmically spaced discrete time shifts are used to calculate full correlation curves from the nanosecond till second time range in a reasonable time.

The correlations are calculated according to equation 2.30 and 2.31. The smallest possible lag time at which a correlation function can be calculated is the pulse spacing between subsequent excitation pulses $\tau_{P}$. First the correlation is calculated for $N$ time shifts $i \tau_{P}$ where $i$ is an integer from 1 to $N$. Secondly, photon time traces are re-binned to a new timebase $\tau_{P}^{\prime}=2 \tau_{P}$ and correlations are calculated for $N$ new time shifts $i \tau_{P}^{\prime}+N \tau_{P}$ where $i$ is again running from 1 to $N$. This whole process of re-binning with a factor of 2 and calculating correlations for $N$ new and larger lag times is repeated until a total delay time of several seconds is reached. The number $N$ is typically a small number such as 10 . 


\subsubsection{Data Fitting}

Data fitting is performed with a Nelder-Meade simplex search algorithm ${ }^{[83]}$. For 2fFCS measurements, ACFs and CCFs are globally fitted against equation 2.54, where velocities $v_{x}$ and $v_{y}$ are set to zero for measurements without flow. The constants $\epsilon_{0} \epsilon_{1}$ in 2.54 are substituted by $\epsilon_{0}^{2}$ when fitting the ACF of the first focus and $\epsilon_{1}^{2}$ for the second focus. Furthermore, the distance $\delta$ can be set to zero, giving the ACFs of the form:

$$
\begin{aligned}
g_{\mathbf{v}}(\tau)=g_{\mathbf{v}}(\infty)+ & \frac{\epsilon_{0}^{2} c \sqrt{\pi}}{2} \int_{0}^{\infty} d \xi \int_{0}^{\infty} d \eta \frac{\kappa(\eta-\sqrt{D \tau} \xi) \kappa(\eta+\sqrt{D \tau} \xi)}{8 D \tau+w^{2}(\eta-\sqrt{D \tau} \xi)+w^{2}(\eta+\sqrt{D \tau} \xi)} \times \\
& \exp \left[-\left(\xi-\frac{v_{z}}{2} \sqrt{\frac{\tau}{D}}\right)^{2}-\frac{2\left(v_{x} \tau\right)^{2}+2\left(v_{y} \tau\right)^{2}}{8 D \tau+w^{2}(\eta-\sqrt{D \tau} \xi)+w^{2}(\eta+\sqrt{D \tau} \xi)}\right]
\end{aligned}
$$

The numerical integration over $\xi$ and $\eta$ has to be re-evaluated for each step of the search.

Data fitting for rotational diffusion measurements is done by first fitting the slow exponential contribution of the Cy5 isomerization in the $\mu$ s time range. These curves were then subtracted from the full correlation curves leaving only the nanosecond contributions of the rotation and photophyics. The remaining curves were fitted with the model from equation 2.45.

\subsubsection{Error Estimation for FCS Measurements}

The error of an FCS measurement can be estimated using a bootstrap method ${ }^{[28]}$. The bootstrap method is fundamentally different from the simpler method where a measurement is split into $N$ shorter measurements, for which the correlation calculations and fittings is done separately, and the standard deviation $\sigma$ is calculated from these results according to

$$
\sigma=\left(\frac{1}{N-1} \sum_{i=1}^{N}\left(X_{i}-\bar{X}\right)^{2}\right)^{1 / 2} .
$$

The problem with this simple approach is that it requires $N$ times longer measurements just to estimate the error without reducing the quality of the correlation curves. Determining the error with this simple method can therefore decrease the fit stability and leads to significant over-estimation of the error if the measurement times are to short.

Using the bootstrap method allows to estimate the standard error without these problems. In the bootstrap method the measurement is first split into $N$ shorter samples for which the correlation curves are calculate separately. In a second step, a number of these subsamples are selected randomly with replacements, meaning that a single subsamples can be included multiple times. The subsamples selected in this way are averaged and form one of the new bootrstrap samples that will be subsequently analyzed. The number of subsamples included in one bootstrap sample is typically equal to the number of subsamples $N$, resulting in a combined measurment time of the bootstrap sample equal to the total measurement time. The second step is repeated, randomly picking a different collection of subsamples each time, to create a large 
number of bootstrap samples. The correlation curves for each of these bootstrap samples are fitted separately, whereupon the error can be estimated according to equation 3.2. 


\section{Results and Discussion}

\subsection{Rotational Diffusion Measurements for Size and Shape Determination of Aldolase}

The rotational diffusion of aldolase, labeled with bis-functional Cy5, was measured according to the scheme for rotational diffusion measurement described earlier. The total measurement time was $5 \mathrm{~h}$. Correlation curves were calculated for lag times ranging from $6.25 \mathrm{~ns}$ to $5 \mu \mathrm{s}$. Exclusion of the value at zero lag-time eliminates the need to include the fluorescence antibunching in the analysis. However, the photo-physics of the dye has to be taken into account. For the dye Cy5, it consist of light induced switching between a fluorescent trans- and a nonfluorescent cis-conformation, and of inter-system crossing between singlet and triplet states. As a result, two characteristic exponential decays are observed in the correlation functions, one slow with ca. $2 \mu$ s decay time, and one fast with ca. $170 \mathrm{~ns}$ decay time. The slow decay was analyzed first by fitting a mono-exponential decay to all curves in the lag-time range from 1 to $5 \mu \mathrm{s}$. This is possible because the fast photo-physical component and rotational contributions to the correlation functions have already completely decayed in this time range. The slow contributions are subtracted from the correlation curves before further processing. The resulting curves are plotted in Figure 4.1.

The reduced correlation curves were then fitted in five different ways: (A) using only the positively valued correlation curves and assuming an isotropic-rotor model, neglecting depolarization effects and assuming collinear absorption/emission dipoles; (B) using all correlation curves and assuming an isotropic-rotor model, again neglecting depolarization effects and assuming collinear absorption/emission dipoles; (C) using all correlation curves and assuming an ellipsoid-of-rotation model; again neglecting depolarization effects and assuming collinear absorption/emission dipoles; (D) using all correlation curves and assuming an ellipsoid-ofrotation model, but now taking into account depolarization effects by the objective $\left(1 / e^{2}\right.$-radius of excitation focus was a priori measured to be $350 \mathrm{~nm}$ ) but keeping absorption/emission dipoles collinear; (E) using all correlation curves, assuming an ellipsoid-of-rotation model, and taking into account depolarization effects and setting the angle between absorption and excitation dipole to $5^{\circ}$ (the approximate angle between excitation and emission dipole for Cy5, see Ref. [45]). The second, short mono-exponential decay was included in these fits, to treat the fast photo-physical relaxation of $\mathrm{Cy} 5^{[112]}$.

Errors were estimated using the bootstrap method described in section 3.3.3. The measurement was split into subsamples of 30 min measurement time, for which correlation curves were calculated separately. For each bootstrap iteration, correlation curves from 5 random subsamples were averaged and fitted. This process was repeated 100 times. The errors of the fit values 


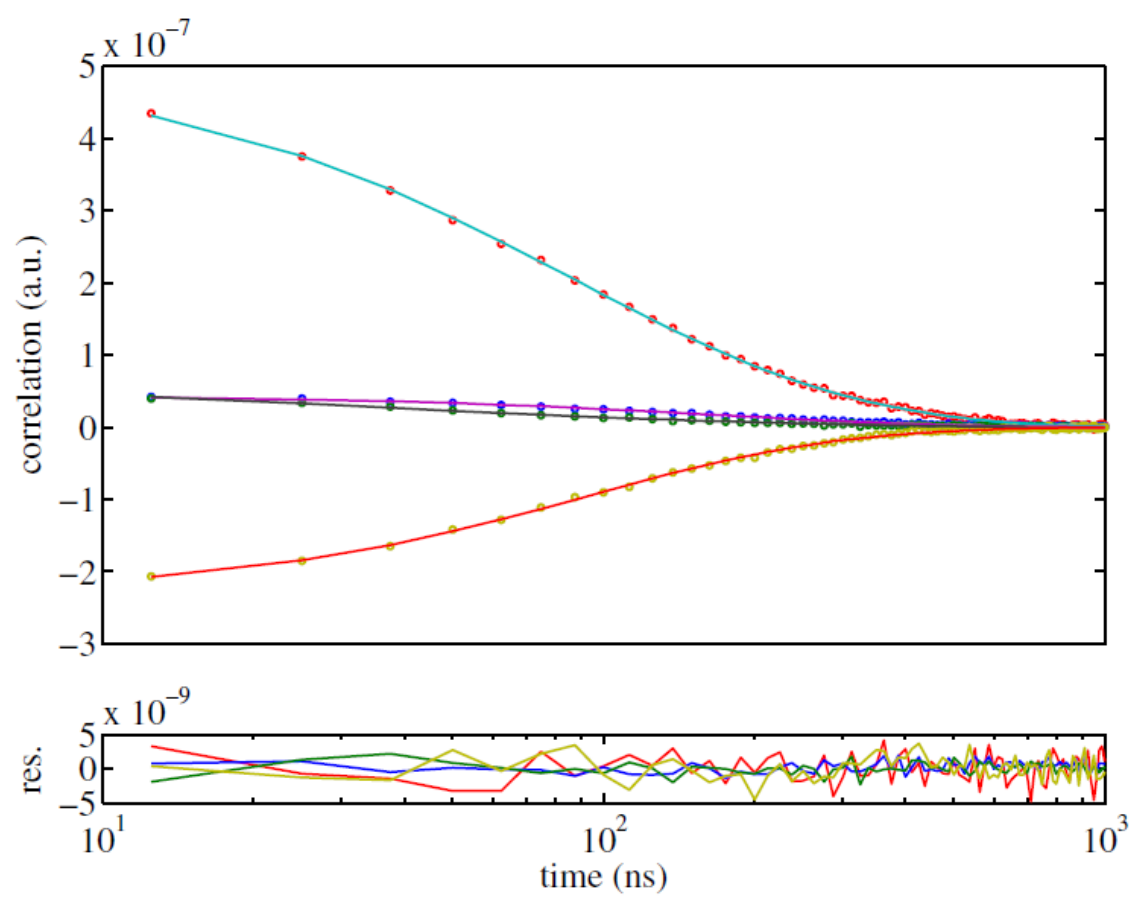

Figure 4.1: FCS measurement result for aldolase (circles). Solid lines show fit result with an anisotropic rotor model.

were then estimated as the standard deviation of all recorded fit results.

The fit results are compared in Table 4.1. The last column indicates the fit quality by presenting the maximum relative residuals. First of all, one notes that the best result is achieved with approach (A): the use of an isotropic rotor model for only the first three correlation curves (those accessible by an experiment with $\mathrm{CW}$ excitation). This result is remarkable as it demonstrates that a conventional FCS experiment wit CW excitation is insufficient to elucidate anisotropic rotational diffusion. However, using pulsed excitation and all four possible correlation curves can determine whether an ellipsoid-of-rotation model fits better than the isotropic-rotor model. The photo-physical relaxation time around $170 \mathrm{~ns}$ is in perfect agreement with values previously reported for Cy5 under similar excitation intensities ${ }^{[12]}$.

To verify the accuracy of the fitted values, the shape of the determined ellipsoid is overlaid with the protein structure known from X-ray crystallography (code 1zah in the Brookhaven Protein Data Bank). The result of the centered and aligned overlay is shown in Figure 4.2, which demonstrates visually how well the measured ellipsoid approximates the actual molecular shape.

These results for rotational diffusion measurements of aldolase show that an FCS experiment with pulsed laser excitation of alternating orthogonal polarization can be used for determining the size and shape of macromolecules. The determined shape of aldolase agrees well to the known crystal structure, within the limits of an ellipsoid-of-rotation model. To determine the 


\begin{tabular}{llcll}
\hline & $R_{1}(\AA)$ & $R_{2}(\AA)$ & $\tau_{\text {isc }}(\mathrm{ns})$ & max res. $(\%)$ \\
\hline $\mathrm{A}$ & - & $39.5 \pm 0.8$ & $166 \pm 33$ & 1.3 \\
$\mathrm{~B}$ & - & $41 \pm 1$ & $173 \pm 18$ & 1.6 \\
$\mathrm{C}$ & $19.8 \pm 0.5$ & $48 \pm 1$ & $170 \pm 8$ & 1.4 \\
$\mathrm{D}$ & $19.7 \pm 0.6$ & $48 \pm 2$ & $168 \pm 16$ & 1.4 \\
$\mathrm{E}$ & $19.8 \pm 0.9$ & $48 \pm 2$ & $169 \pm 16$ & 1.4 \\
\hline
\end{tabular}

Table 4.1: Results of the FCS data fitting. Capital letters indicate which kind of data fitting was applied; (A) fit with an isotropic rotor model, using only the correlation curves accessible with a cw-measurement; (B) Fit with an isotropic rotor model, using all four correlation curves; (C) Fit with a rotational ellipsoid model while neglecting depolarization by the high-NA objective and assuming collinearity between dye absorption and emission dipole; (D) Same as C, but taking depolarization effects into account for a focal $1 / e^{2}$-radius of $350 \mathrm{~nm}$; (E) same as D, but assuming an angle between absorption and emission dipole of $10^{\circ}$. Second column: radius value of the symmetry axis of the rotational ellipsoid (for isotropic rotor, the sphere radius is given in the next column). Third column: radius value of orthogonal axes of rotational ellipsoid. Fourth column: photophysics relaxation time. Fifth column: maximum relative residual of fit.
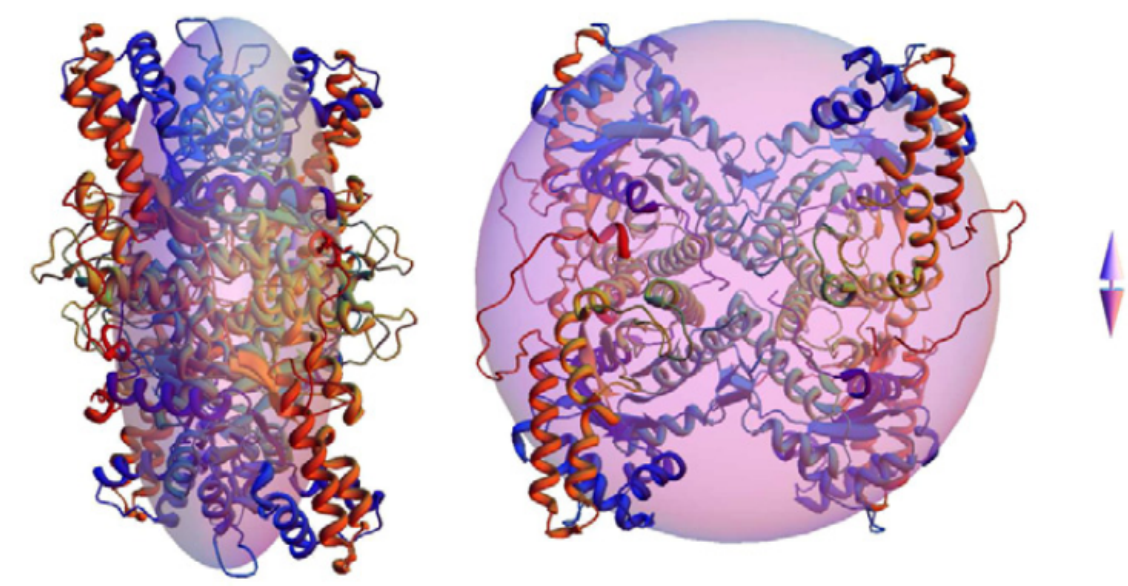

Figure 4.2: Overlay of aldolase structure as determined from X-ray scattering with rotational ellipsoid as determined with FCS. Left: view along the one of the two long ellipsoid axes. Right: view along the short rotational symmetry axis of the ellipsoid. Double arrow has $1 \mathrm{~nm}$ length. 
shape all possible correlation curves must be recorded. This can be easily achieved, in a single measurement, with the simultaneous use of four single-photon detectors. Measuring all possible correlations with only two detectors is also possible but requires several consecutive measurements with different detector configurations.

\subsection{Diffusion of Aldolase in Crowded Environments}

The diffusion of molecules in crowded solutions was systematically investigated for different sizes of molecules, different types of diffusion, and at varying crowder concentrations. A solution of Polyethylene Glycol (PEG) in Phosphate Buffered Saline (PBS) at pH 7.4 and physiological salt concentrations was used as a model system of a crowded environment. PEG in two different sizes ( $2 \mathrm{kDa}$, PEG_2 and $25 \mathrm{kDa}$, PEG_25) was used to investigate the influence of crowder size on the diffusive behavior of the molecules. PEG concentration varied from zero to 30 weight percent for PEG_2, and from zero to 20 weight percent for PEG_25. For all conditions, the translational diffusion of the macromolecule aldolase and the small dye Atto 655 was measured by $2 \mathrm{fFCS}$. Additionally, the rotational diffusion of aldolase was determined by FCS on the nanosecond time scale. As a reference, the macroscopic kinematic viscosity of different PEG solutions was measured with an Ubbelohde viscometer.

The measured diffusion coefficients for aldolase in PEG_25 solutions are depicted in Figure 4.3. The results show an exponential dependence of the diffusion coefficient on PEG concentration (solid line in the graph). The rotational diffusion times of aldolase in PEG_25 solutions determined by FCS, are plotted in Figure 4.4. The dependence of the rotational diffusion times on the PEG concentration is equally well described by an exponential function.

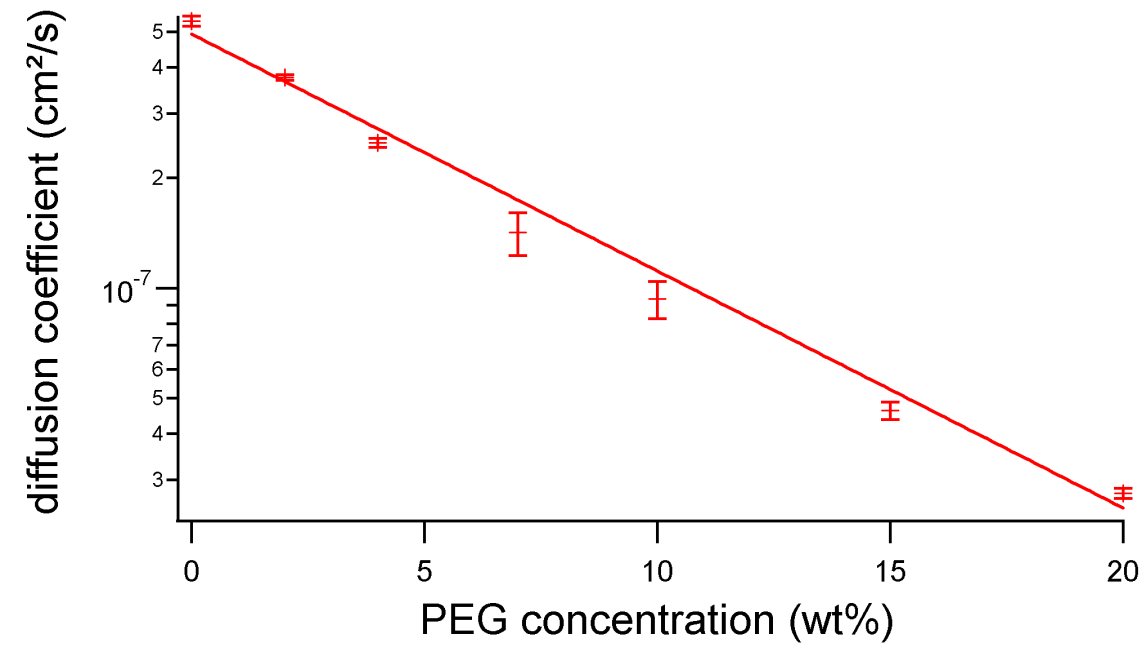

Figure 4.3: Diffusion coefficient of aldolase in PEG_25 (25 kDa MW PEG) for different concentrations measured with $2 \mathrm{fFCS}$

To relate the measurements of aldolase diffusion to the macroscopic viscosity measurements, the Stokes-Einstein relation can be used. If the hydrodynamic radius of a molecule is known, 


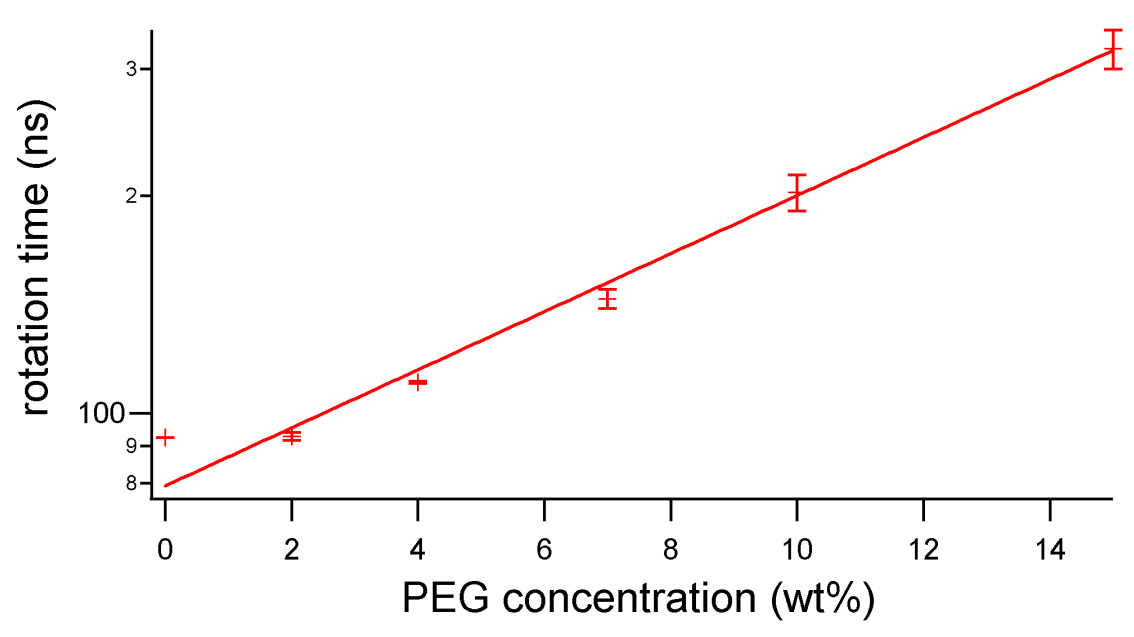

Figure 4.4: Rotation time of aldolase in PEG_25 (25 kDa MW PEG) for different concentrations measured with nanosecond timescale FCS

the viscosity of the solution can be calculated from the measured diffusion coefficient using equation 2.12. Hence, if the diffusion coefficient of a molecule was measured in a solution of known viscosity $\eta_{0}$, the viscosity $\eta$ of a second solution can be determined from a diffusion measurement with the same molecule. One obtains the relation:

$$
\frac{\eta}{\eta_{0}}=\frac{D_{0}}{D}
$$

where $D_{0}$ is the diffusion coefficient of the molecules in the solution of known viscosity and $D$ the diffusion coefficient of the molecule in the second solution. Similarly, for rotational diffusion times one obtains

$$
\frac{\eta}{\eta_{0}}=\frac{T}{T_{0}}
$$

where $T_{0}$ is the rotational diffusion time in a solution of known viscosity and $T$ the rotational diffusion time in the solution with unknown viscosity.

The viscosity values determined from translational diffusion measurements of aldolase in PEG_25 are shown as red circles in Figure 4.5. Macroscopic viscosities are illustrated as black triangles and viscosity values determined from diffusion measurement of the dye Atto 655 are depicted as green squares. The blue crosses represent the viscosity values determined from rotational diffusion measurements of aldolase.

The viscosity value calculated from the diffusion measurements of the small molecule Atto 655 is much smaller than the one determined by macroscopic means. This is in agreement with previously published diffusion measurements of small particles in polymer solutions. Small particles can diffuse much faster than the diffusion speed calculated from their size and the macroscopic viscosity of the polymer solution ${ }^{[99,85]}$. The viscosity values determined from the measurements of the much larger molecule aldolase show a similar dependence on the 
concentration but are not reduced as strongly as those determined for Atto 655. Interestingly, viscosity values determined from the rotational diffusion measurements of aldolase differ from those determined from translational diffusion measurements. The viscosities determined from rotational diffusion measurements are smaller than those determined from translational diffusion measurements for all PEG concentrations. The difference increases with increasing PEG concentration. In fact, the viscosities determined from the rotational diffusion measurements of aldolase are closer to the values determined from translational diffusion of the small dye molecule.

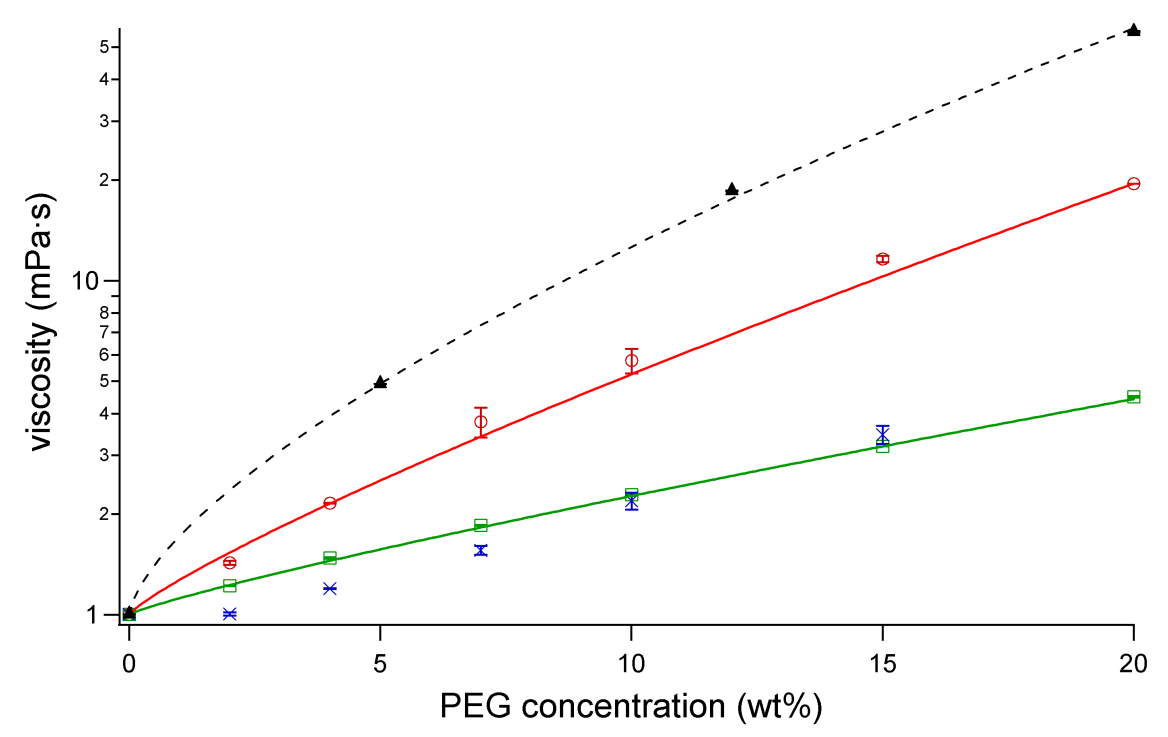

Figure 4.5: Effective viscosity PEG_25 (25 kDa MW PEG) solutions as a function of concentration. Macroscopic viscosity measured with an Ubbelohde viscometer (black triangles); viscosity calculated from 2fFCS measurements of aldolase (red circles); viscosity calculated from 2fFCS measurement of small dye ATTO 655 (green squares); viscosity calculated from rotational diffusion measurement of aldolase (blue crosses)

The viscosity values are best fitted with a stretched exponential function:

$$
\eta=\eta \exp \left(a c^{\nu}\right)
$$

where $c$ is the PEG concentration, and $v$ is a scaling parameter close to one. The results for the different fits (lines in Fig. 4.5) are listed in Table 4.2. The values observed for the scaling parameter $v$, obtained from translational diffusion measurements, are similar to the value for diffusion of gold nanoparticles in polystyrene solutions reported previously by Omari et al. ${ }^{[55]}$.

The viscosity values determined from measurements in solutions of the shorter PEG_2 (2 $\mathrm{kDa}$ ) are depicted in Figure 4.6. In comparison to the results from the PEG_25 measurements (fig. 4.5) there are some notable differences. First, the difference between the two types of measurement is much less pronounced. Second, the data points fit very well with an exponential curve (scaling parameter $v=1$ ). The values obtained from translational diffusion measurements of aldolase are in excellent agreement with the macroscopic viscosities (black triangles). 


\begin{tabular}{lll}
\hline & $a$ & $v$ \\
\hline Macroscopic & 0.538 & 0.67 \\
Aldolase translation & 0.238 & 0.84 \\
Atto 655 translation & 0.112 & 0.86 \\
Aldolase rotation & 0.07 & 1.11 \\
\hline
\end{tabular}

Table 4.2: Fit results for concentration dependent viscosity curves.

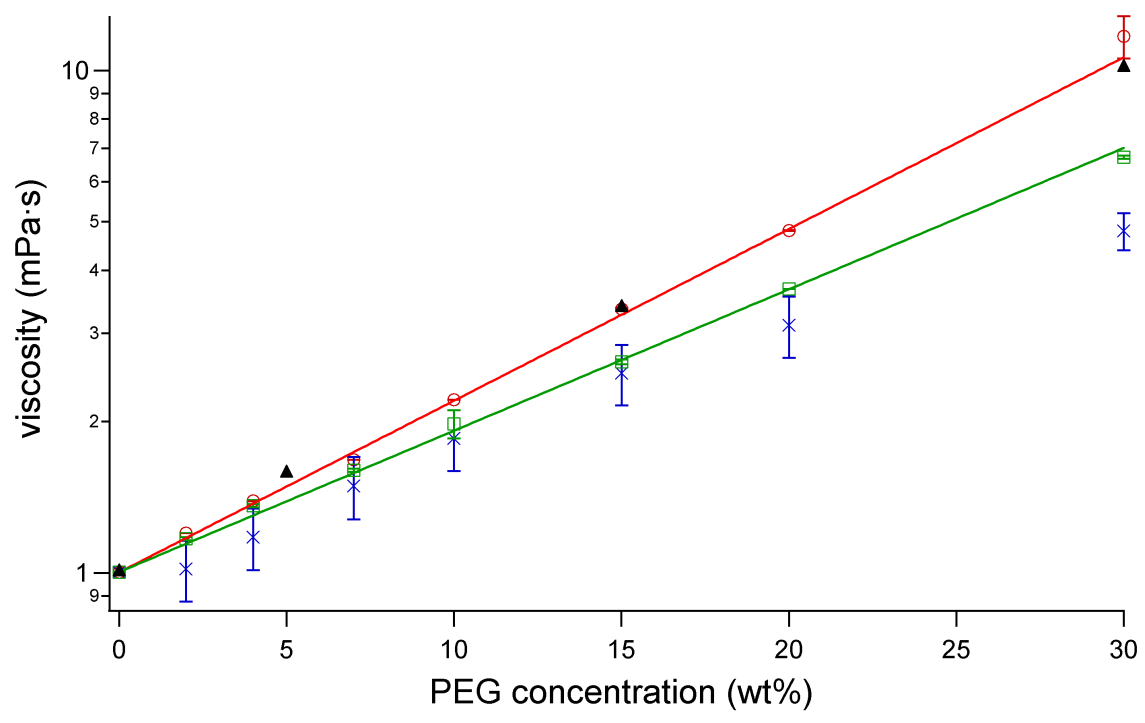

Figure 4.6: Effective viscosity of PEG_2 (2 kDa MW PEG) solutions as a function of concentration. Macroscopic viscosity measured with an Ubbelohde viscometer (black triangles); viscosity calculated from 2fFCS measurements of aldolase (red circles); viscosity calculated from 2fFCS measurement of small dye ATTO 655 (green squares); viscosity calculated from rotational diffusion measurement of aldolase (blue crosses) 
The values obtained from the diffusion measurement of Atto 655 are still significantly lower than those obtained from aldolase diffusion and macroscopic measurements. Another similarity to the measurements with PEG_25 is the apparently lower viscosity determined from the rotational diffusion measurement of aldolase.

These results confirm the assumption that diffusion in a crowded solution is influenced by at least three factors: the size of the crowder, the concentration of the crowder, and the size of the diffusing molecule. Furthermore, the diffusion coefficients and viscosity depend exponentially on the PEG concentration, and therefore this dependence is very different from the one predicted by Einstein for a suspension of hard spheres ${ }^{[31]}$ :

$$
\eta=\eta_{0}(1+2.5 \Phi)
$$

where $\eta_{0}$ is the solvent viscosity and $\Phi$ the volume fraction occupied by the spheres. Clearly a solution of flexible polymers behaves differently from a suspension of hard particles. A comparison of the size of the polymer chains in solution and the size of the diffusing molecules can improve the understanding of diffusion in crowded solutions. The size of a polymer is well described by its radius of gyration, which is the root mean square distance of its parts from the center. For PEG of known molecular weight $M_{w}$ the radius of gyration $R_{g}$ can be calculated according to ${ }^{[25]}$ :

$$
R_{g}=0.215 M_{w}^{0.583 \AA} \text {. }
$$

For the PEGs used here a radius of $18 \AA$ for the small PEG and a radius of $78 \AA$ for the larger PEG is calculated. The hydrodynamic radii of the molecules measured are $39.5 \AA$ for aldolase (see section 4.1) and 5.9 $\AA$ for Atto 655. Theoretically, the viscosity determined from diffusion coefficients should correspond to the macroscopic measurement when the molecule is much larger than the radius of gyration of the polymer. This is experimentally confirmed, by the viscosity calculated from the diffusion measurements of aldolase in the PEG_2 solution. Even though, in this case, the molecule (aldolase) is only twice the size of the PEG_2's radius of gyration, the calculated viscosity matches the macroscopic one. Hence, the solution appears to influence the diffusion as if it was a continuous fluid.

In all other measurements, the molecules are smaller than the radius of gyration of the PEG used. The assumption of a continuous viscous medium is incorrect, in these cases. Here, the molecule is diffusing through a meshwork of other larger molecules while still finding holes and gaps that are mostly filled with solvent. As a consequence, the viscosity values presented in Figure 4.5 and 4.6 have to be interpreted as effective viscosities. Another unexpected result is the difference in the effective viscosities calculated from the rotational and translational diffusion measurements of aldolase. This can be explained by the fact that rotational diffusion is much faster than the translational diffusion on the length scale which is probed with $2 \mathrm{fFCS}$. The distance of the two foci in the experiment was $450 \mathrm{~nm}$ leading to diffusion times on the order of tens to hundreds of milliseconds, while rotational diffusion times are on the order of hundreds of nanoseconds. The environment that influences rotational diffusion is more localized, as the molecule cannot move very far in the short time typical for rotational diffusion. The environment directly adjacent to the molecule is characterized by a depletion layer, a 
thin layer of solvent where polymer concentration is lower than the average concentration throughout the solution (due to loss of configurational entropy) ${ }^{[7,101,35]}$. As rotational diffusion occurs mostly without displacement of a molecule, the influence of the crowder is smaller than on translational diffusion. These results are in good agreement with previous theoretical ${ }^{[35]}$ and experimental ${ }^{[50]}$ studies.

\subsection{Translational and Rotational Diffusion of alphaB-Crystallin}

As a second model system for molecular crowding, the (self)-diffusion of the small heat shock protein alphaB-crystallin $(\alpha \mathrm{B})(\sim 20 \mathrm{kDa})$ was investigated at high protein concentrations. $\mathrm{Pu}-$ rified $\alpha \mathrm{B}$ was kindly provided by Bernd Reif (Leibniz-Institut für Molekulare Pharmakologie (FMP), Berlin, Germany). In vivo, $\alpha \mathrm{B}$ assembles into high-molecular-weight complexes of approximately $600 \mathrm{kDa}^{[80]}$. As a small heat shock protein, $\alpha \mathrm{B}$ can act as a chaperone and prevents aggregation of small amyloid proteins ${ }^{[51,98,82]} \cdot \alpha \mathrm{B}$ (and $\alpha \mathrm{A}$ ) are major constituents of the human eye lens, accounting for approximately $30 \%$ of the total protein mass. Crystallins are highly soluble and do not aggregate even at protein concentrations up to $400 \mathrm{mg} / \mathrm{ml}$. This property is essential for the high refractive index and the optical transparency of the eye lens.

For the diffusion measurements, a small part of $\alpha \mathrm{B}$ labeled with bis-functional Cy5 was mixed with solutions of unlabeled $\alpha \mathrm{B}$, of different concentrations up to $300 \mathrm{mg} / \mathrm{ml}$. Translational diffusion measurements by Dual Focus Fluorescence Correlation Spectroscopy (2fFCS) and rotational diffusion measurements by nanosecond time-scale FCS were performed as described earlier. The diffusion coefficient of $\alpha \mathrm{B}$ determined at approx. $1 \mathrm{nM}$ concentration corresponds to a hydrodynamic radius of $6.61 \mathrm{~nm}$. This size is consistent with the calculated molecular weight of complexes of $600 \mathrm{kDa}$ and agrees well with previously published values measured by DLS and gel electrophoresis ${ }^{[52,77]}$.

The diffusion coefficients determined by 2fFCS for different concentrations of $\alpha \mathrm{B}$ are depicted as crosses in Figure 4.7. For concentrations up to approximately $100 \mathrm{mg} / \mathrm{ml}$ the diffusion coefficients can be fitted well by a mono-exponential function (dashed line). For the higher concentrations (approx. from $150 \mathrm{mg} / \mathrm{ml}$ on) the diffusion coefficients decrease stronger.

The rotational diffusion times for the same concentrations of $\alpha \mathrm{B}$ are shown in Figure 4.8. The dependence of the rotation times on the protein concentration is well described by a single exponential function for concentrations up to $\sim 250 \mathrm{mg} / \mathrm{ml}$ (dashed line).

The effective viscosities determined from translational and rotational diffuion measurements are plotted in Figure 4.9. It is important to note, that these values where calculated under the assumption of a fixed size of crystallin clusters. Both datasets can be described by a stretched exponential up to protein concentrations of $\sim 250 \mathrm{mg} / \mathrm{ml}$ (dashed lines). At the highest protein concentration there is a significant jump in viscosities determined by both types of measurement.

While some characteristics, such as the lower viscosity values determined from rotational 


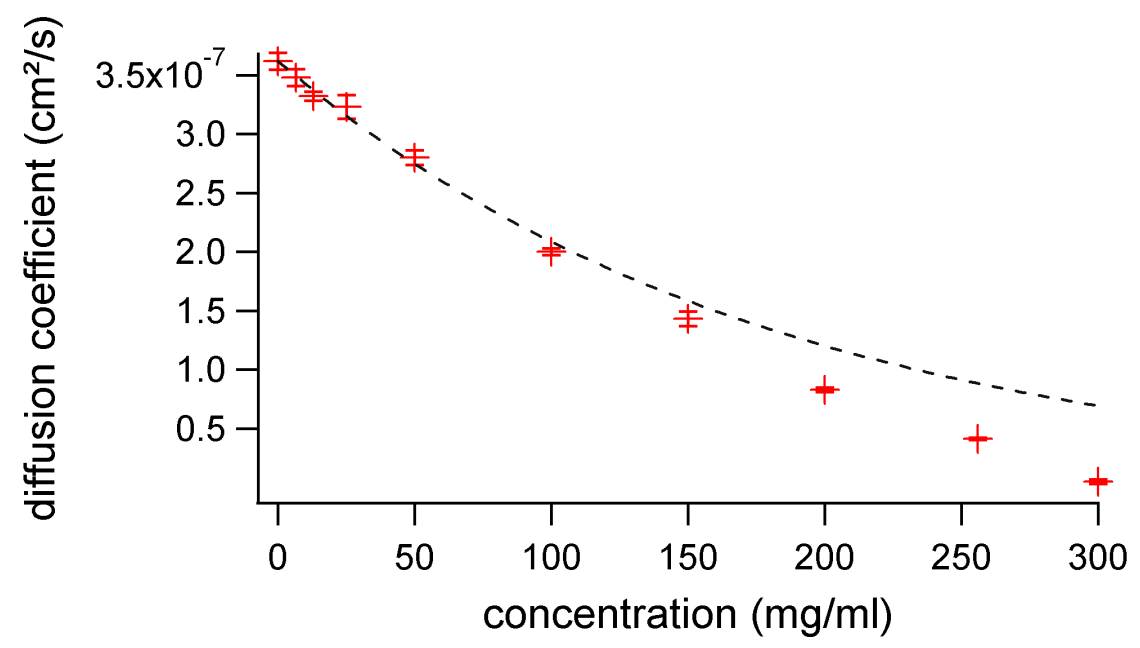

Figure 4.7: Diffusion coefficient of $\alpha \mathrm{B}$ Crystallin for different concentrations of crystallin measured with 2fFCS. Dashed line is fit with a mono-exponential function.

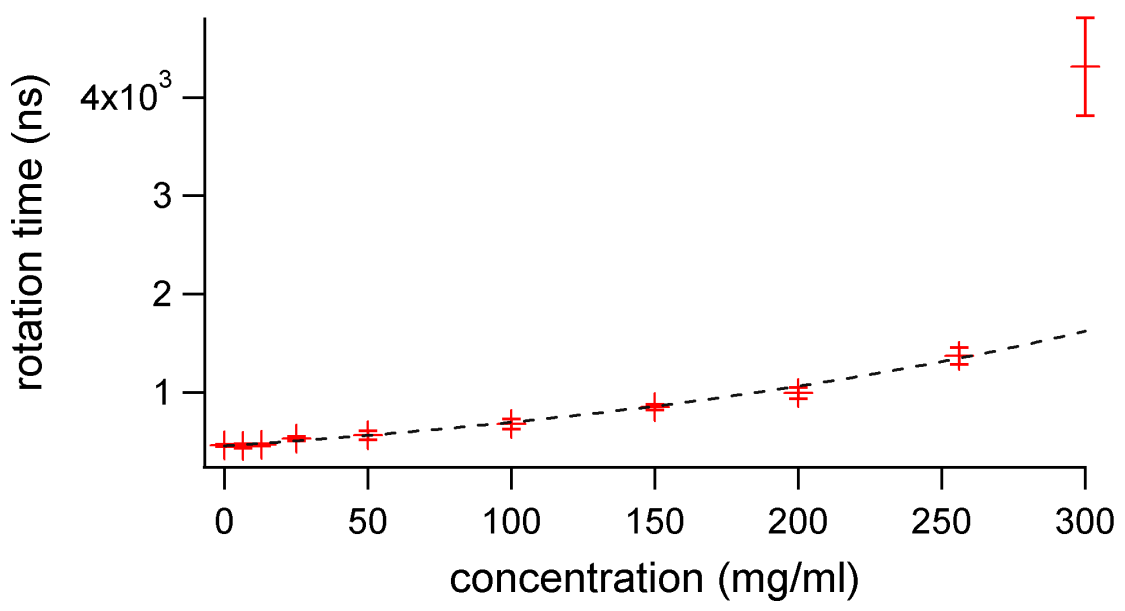

Figure 4.8: Rotational diffusion times of $\alpha \mathrm{B}$ Crystallin for different concentrations of crystallin measured with nanosecond time-scale FCS. Dashed line is fit with a monoexponential function. 


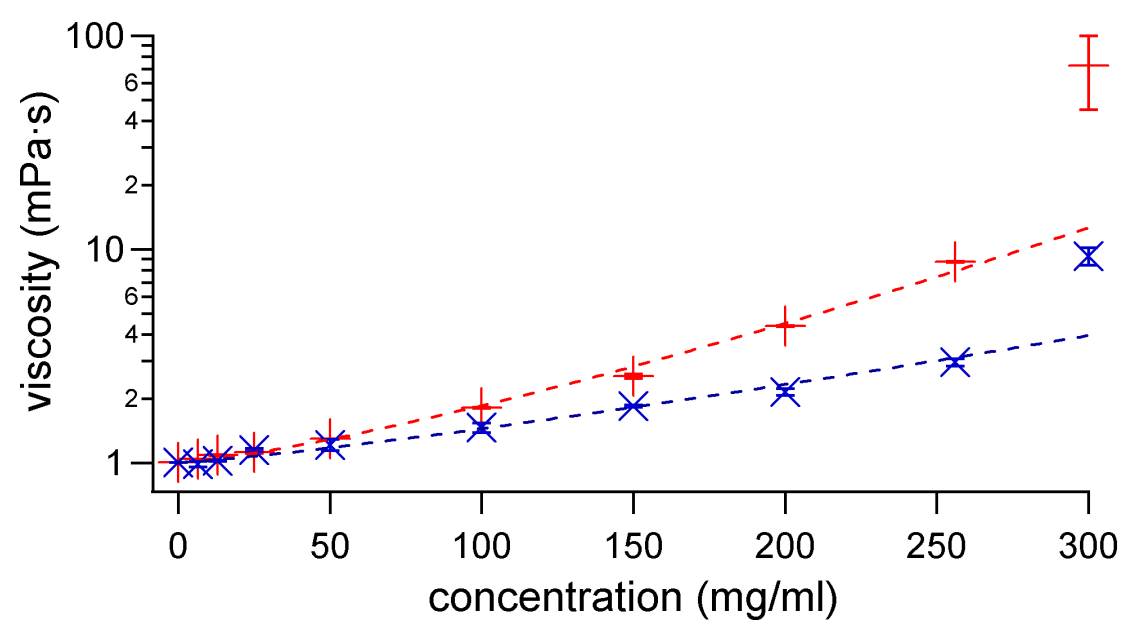

Figure 4.9: Effective viscosity of different concentration $\alpha$ B-Crystallin solutions. Viscosity calculated from 2fFCS measurements (red '+'); viscosity calculated from rotation measurement (blue ' $x$ '). Dashed lines are fits with a stretched exponential function.

diffusion, are similar to the measurements in PEG solutions (section 4.2) there are some fundamental differences. The scaling parameter $v$ is smaller than one for PEG solutions, whereas it is larger than one for solutions of $\alpha \mathrm{B}$-Crystallin. In the case of $\alpha \mathrm{B}$ diffusion, the viscosity values determined from translation and rotation measurements coincide up to concentrations of $25 \mathrm{mg} / \mathrm{ml}$, and diverge for higher concentrations. Finally, there is a distinct jump in viscosity at very high concentrations of $\alpha \mathrm{B}$.

A plausible explanation for the difference between a solution of PEG molecules and a solution of $\alpha \mathrm{B}$ is the difference in the type of interactions of the respective molecules. While there is some interaction of PEG molecules in aqueous solution ${ }^{[25]}$, there is a strong attractive interaction between $\alpha \mathrm{B}$ molecules, as they can bind to each other and form clusters of approximately 30 molecules $^{[80]}$. The strong interactions of crystallins can lead to increased clustering at high protein concentrations ${ }^{[4,62,113]}$. The jump in viscosities at high protein concentrations is either due to an overall transition to a gel-like phase or due to sudden increase in $\alpha \mathrm{B}$ cluster size.

\subsection{Measurement of Liquid Crystal Flow Profiles in Microfluidic Channels}

For flow measurements in microfluidic channels, the liquid crystal 5CB (fig. 3.4) was used. Microfluidic chips and liquid crystals were kindly provided by Christian Bahr (Max-PlanckInstitute for Dynamics and Self-Organization, Göttingen, Germany).

All 2fFCS measurements were performed with the channel aligned parallel to the connecting line of the two foci of the 2fFCS system (see fig. 4.10a). Exemplary correlation curves 
calculated from a measurement of 5CB flow in a channel with rectangular profile and without surface anchoring are shown in Figure 4.10b. The determined flow velocity is $124.1 \mu \mathrm{m} / \mathrm{s}$.

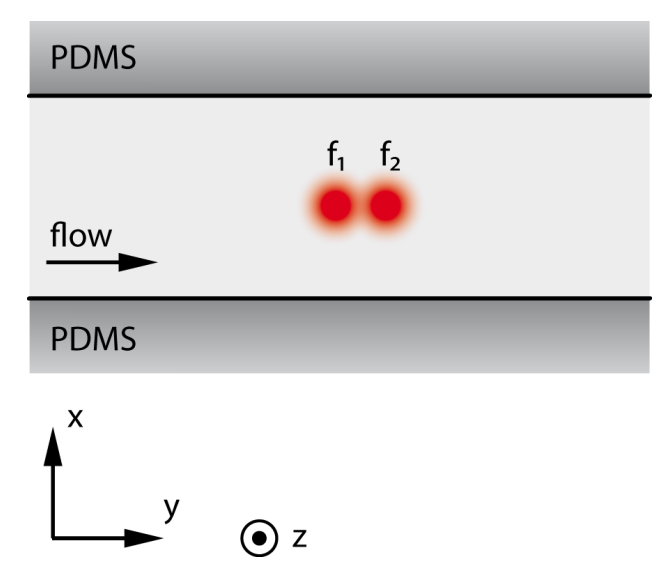

(a)

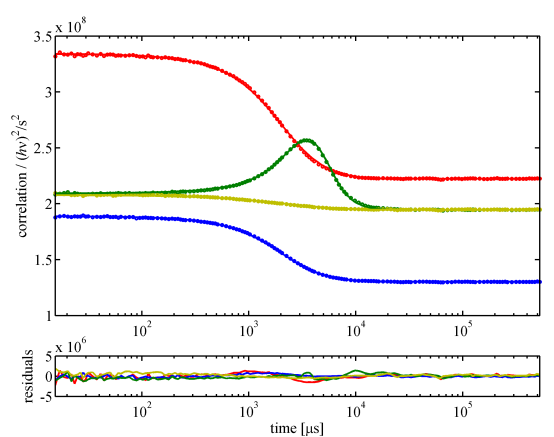

(b)

Figure 4.10: (a): Schematic illustration of the alignment of the two foci with respect to the microfluidic channel (top view). (b): Typical correlation curves of a 2fFCS measurement of liquid crystal flow with fits (solid lines). The four curves are comprised of two autocorrelation curves (one from each focus, red and blue), the forward cross-correlation $\left(f_{1} \rightarrow f_{2}\right.$, green), and the backward cross-correlation $\left(f_{2} \rightarrow f_{1}\right.$, yellow).

The flow profile in this channel was measured on a grid of 45 by 5 points in a plane perpendicular to the direction of the flow ( $x-z$ plane). The spacing of points was $1.333 \mu \mathrm{m}$ along the $x$-axis and $3 \mu \mathrm{m}$ along the $z$-axis. The measurement time in each point was 5 minutes at a volume flow rate of $0.025 \mu \mathrm{l} / \mathrm{min}$. Figure 4.11 shows the determined velocity values. The solid lines show a global fit of all velocity values with the theoretical prediction for a laminar flow in a rectangular duct ${ }^{[49]}$.

In general, the measured values agree well with the theoretical flow velocities of an isotropic fluid in a rectangular duct. The results show that the velocity profile of 5CB flow is well described by this model under the chosen experimental conditions (low flow rate, no surface anchoring).

Even though 5CB behaves similar to an isotropic fluid at low flow rates, in general aligned nematic liquid crystals show a strong anisotropy in their viscosity ${ }^{[60,8]}$. As a result, nematic liquid crystals show an alignment of their molecular axis with the flow ${ }^{[70,12]}$. This is the reason for the ER defect described in the introduction. Here, a microfluidic channel with a cylindrical pillar was used to study the flow profile through such a defect (see fig. 4.12). The pillar helped to stabilize the defect at low flow rates.

The velocities were measured along two different lines parallel to the $x$-axis and $100 \mu \mathrm{m}$ downstream of the pillar (red line in Fig. 4.12a). The resulting flow velocities are plotted in figure 4.13. 


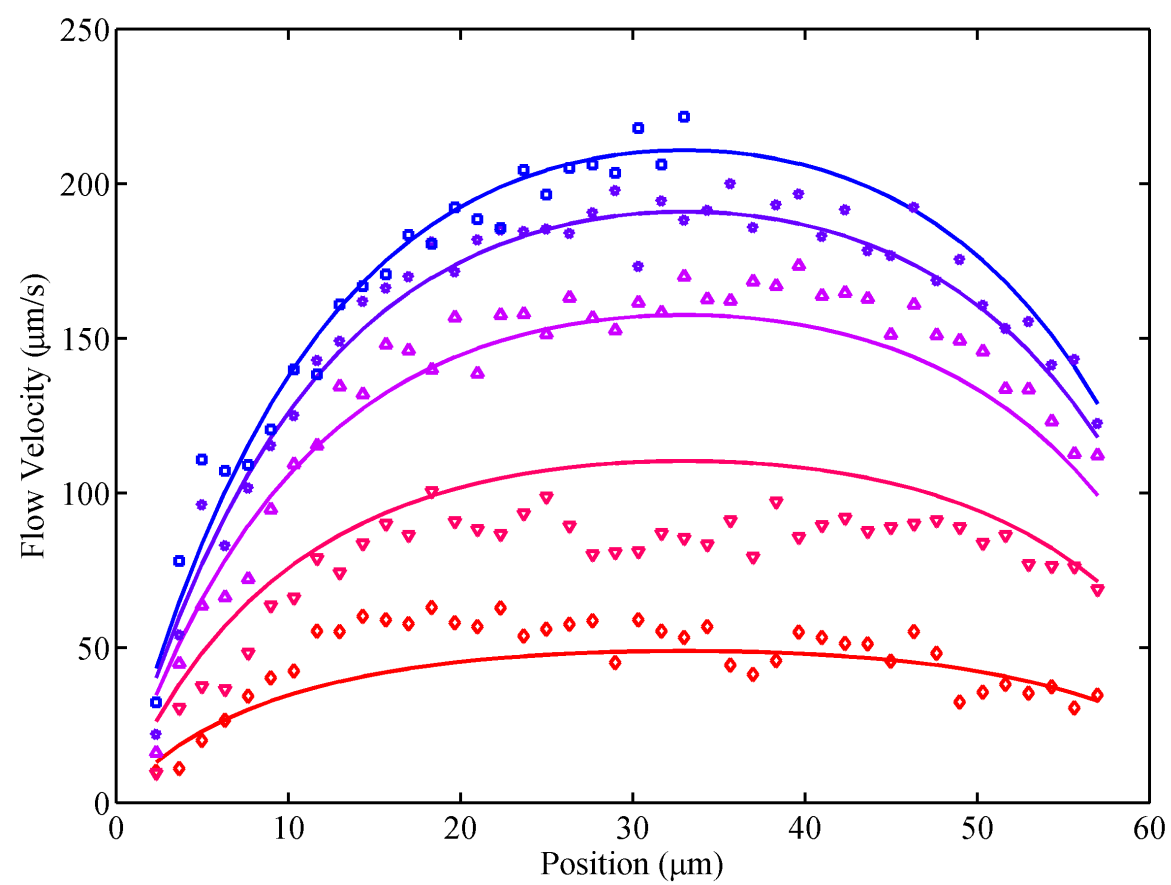

Figure 4.11: Flow velocity of $5 \mathrm{CB}$ in a microfluidic channel with rectangular profile at a volume flow rate of $0.025 \mu \mathrm{l} / \mathrm{min}$. Z-Position increases from red to blue in steps of $3 \mu \mathrm{m}$. Solid curves are the fitted velocity profile in a rectangular duct with dimensions $66 \mu \mathrm{m} \times 34 \mu \mathrm{m}$

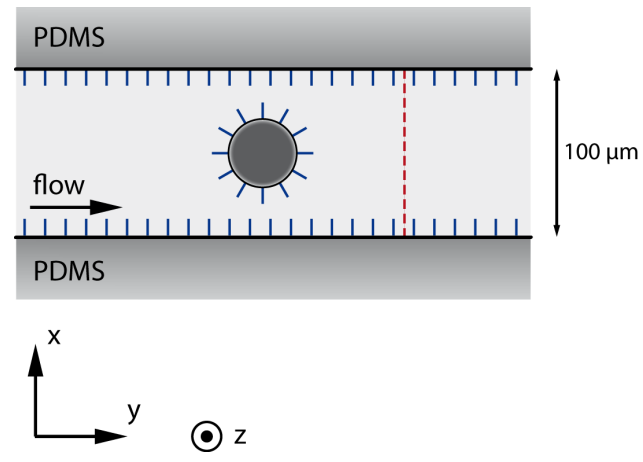

(a)
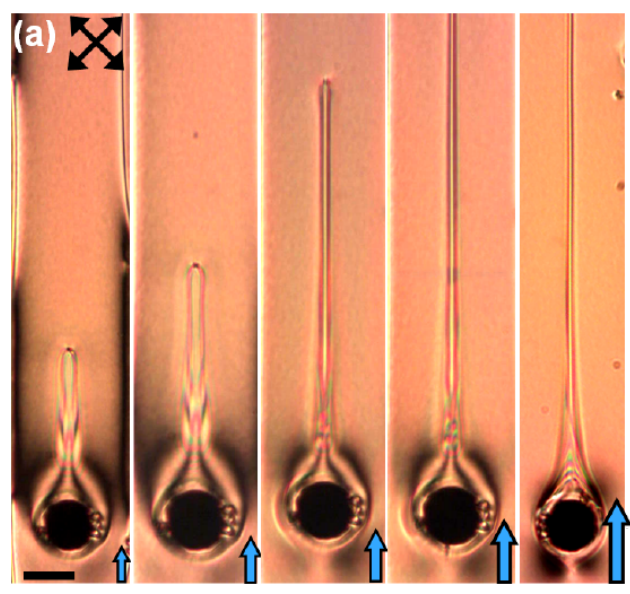

(b)

Figure 4.12: (a): Schematic representation of the microfluidic channel with a cylindrical pillar (top view). The homeotropic surface anchoring is indicated by the blue lines. Flow velocities were recorded along the red line. (b): Images of the defect forming after the pillar with increasing flow rates (increasing rates indicated by the size of the blue arrows; images courtesy of Anupam Sengupta, MPI for Dynamics and Self-Organization, Göttingen). 


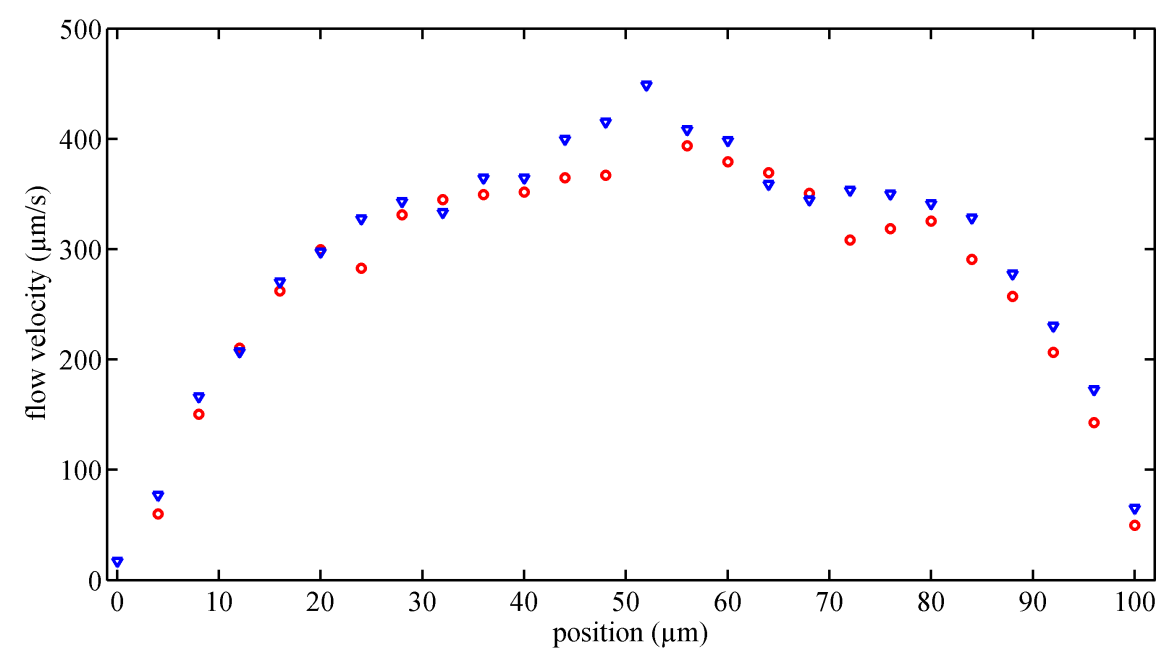

Figure 4.13: Flow velocity of 5CB in a microfluidic channel with rectangular profile and central cylindrical pillar at a volume flow rate of $0.2 \mu \mathrm{l} / \mathrm{min}$. The flow profile was measured $100 \mu \mathrm{m}$ after the pillar in the direction of the flow and $15 \mu \mathrm{m}$ above the cover slide surface. The dimensions of the channel are $100 \mu \mathrm{m} \times 35 \mu \mathrm{m}$. The channel was treated for homeotropic surface anchoring.

The flow velocity profile through the defect (Fig. 4.13) is quite different from the velocity profiles measured for normal flow (Fig. 4.11). It shows a higher flow velocity at the center of the channel, where the defect is located. To ensure that the measured increase was due to the defect, a control measurement was performed in front of the pillar at the same flow rate. The flow profile in front of the pillar shows no higher velocity at the center of the channel (Fig. 4.14). To verify that the higher flow velocity is an effect of the liquid crystal, a second control measurement was performed $100 \mu \mathrm{m}$ after the pillar, but using a glycerol solution (Fig. 4.15). The measurement with the glycerol solution shows no higher flow velocity at the center of the channel. Thus, the higher flow velocity is due to the anisotropy of the liquid crystal and the specific ordering in and around the defect. Aligned nematic liquid crystals have a much lower viscosity when sheared parallel to their long molecular axis ${ }^{[60,8]}$. The molecules inside such a defect are aligned along the direction of the flow. As a result, flow velocities inside the defect are higher than outside.

In conclusion, these results clearly demonstrate that $2 \mathrm{fFCS}$ can be used to accurately measure flow velocities, even inside complex anisotropic fluids. For the first time, the flow velocity across a defect line in a nematic liquid crystal was measured with high spatial resolution. The resulting flow profile shows a higher flow velocity inside the defect, a phenomenon which is impossible in isotropic liquids. 


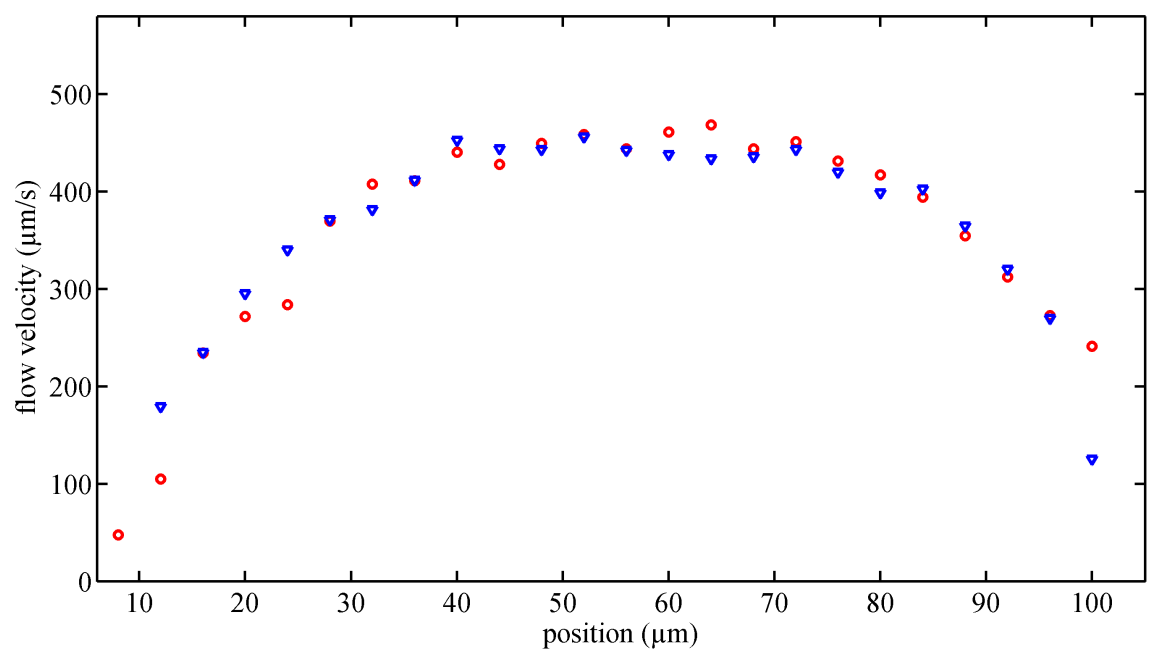

Figure 4.14: Control measurement without pillar. Flow velocity of 5CB in a microfluidic channel with rectangular profile and central cylindrical pillar at a volume flow rate of $0.2 \mu \mathrm{l} / \mathrm{min}$. The flow profile was measured several $\mathrm{mm}$ before the pillar in the direction of the flow and $15 \mu \mathrm{m}$ above the cover slide surface.

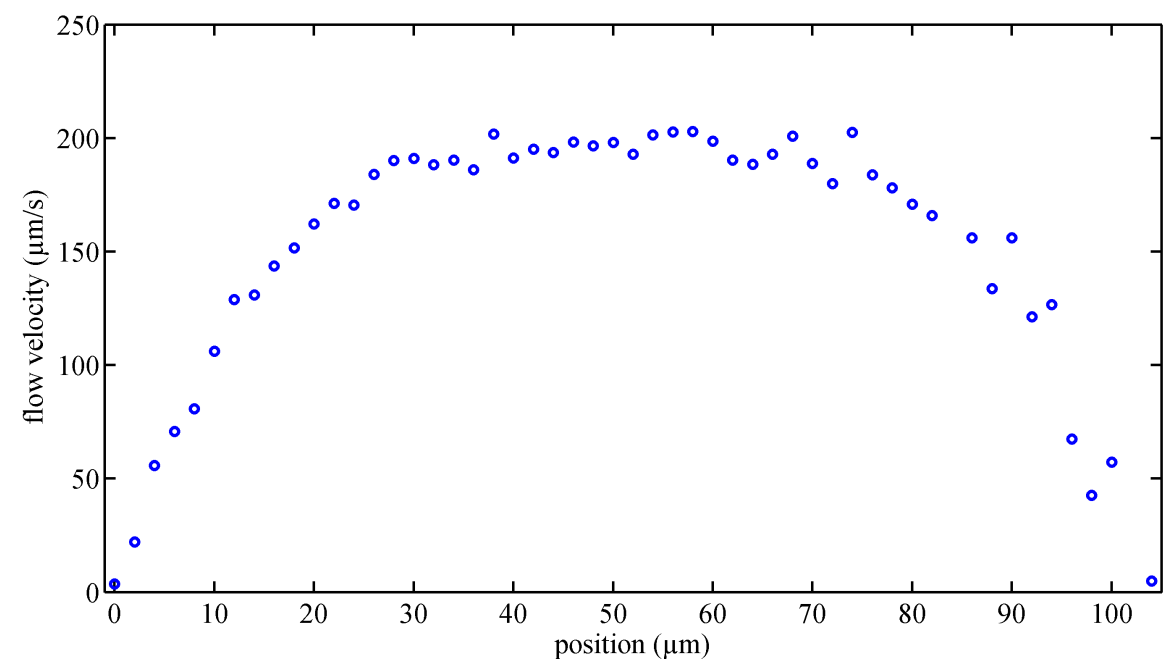

Figure 4.15: Control measurement with glycerol. Flow velocity in a microfluidic channel with rectangular profile and central cylindrical pillar at $0.2 \mu \mathrm{l} / \mathrm{min}$ volume flow rate of 73 wt $\%$ glycerol. The flow profile was measured $100 \mu \mathrm{m}$ after the pillar in the direction of the flow and $15 \mu \mathrm{m}$ above the cover slide surface. 



\section{Conclusions}

The work presented in this thesis clearly display the use of fluorescence correlation spectroscopy for studying the physics of fluids on microscopic length scales. Dual focus FCS was used for direct measurements of flow profiles in microfluidic devices with high spatial resolution. Importantly, for the first time ever, a local increase in flow velocity inside a defect line of an aligned nematic liquid crystal was observed.

The presented measurements show that FCS can be used for rotational diffusion measurements from tens of nanoseconds up to several microseconds. At longer rotation times, it becomes more difficult to separate between the contributions of the rotational diffusion and the ones resulting from dye photo physics to the fluorescence correlation curves. The use of fluorescent dyes with no triplet state dynamics, or cis-trans isomerization can extend the time range of rotational diffusion measurements. So far only few dyes with bis-functional modification are available. If other fluorescent dyes, such as Atto 655, become available with this modification, the accuracy and time range of rotational diffusion measurements could be increased much further. However, the available time range is ideally suited for measuring the rotational diffusion of macromolecules. Here, it is shown that, not only is it possible to exactly determine the size of a molecule using FCS, but also to extract its general shape with an ellipsoid of rotation model.

Furthermore, both $2 \mathrm{fFCS}$ and FCS for rotational diffusion measurements can be used to probe the local viscosity of fluids and crowded solutions. With dual focus FCS the translational diffusion coefficient of a fluorescent molecule can be determined accurately, even when the refractive index of the solution varies. This is exemplified by the precise agreement of viscosities determined from aldolase diffusion in PEG_2 solutions with the viscosities determined by macroscopic measurements. These results show that, even at polymer concentrations as high as $300 \mathrm{mg} / \mathrm{ml}$, diffusion coefficients determined by dual focus FCS are accurate. Additionally, the viscosity of a solution can be probed on a shorter time- and length scale by measuring the rotational diffusion of a molecule. Measuring the translational diffusion of molecules with different sizes and their rotational diffusion in crowded solutions contributes to the understanding of the physical properties of such environments.

The measurements of diffusion in crowded environments presented in this work confirm several of the key properties attributed to these complex solutions. It was confirmed that an effective viscosity of a crowded solution, depending strongly on the size of the molecule, has to be used. This is in good agreement with previous reports on the size dependent viscosity of crowded solutions ${ }^{[50,22]}$. Furthermore, it proves that the behavior of a crowded solution on microscopic length scales can not be sufficiently described by a single continuum viscosity. This work showed that, for a molecule twice as large as the radius of gyration of the crowder, the viscosity affecting translational diffusion is equal to the macroscopic viscosity. For smaller 
molecules the effective viscosity is closer to the viscosity of the solvent than the effective viscosity of macromolecules. An increase in crowder concentration influences the effective viscosity for molecules of different size in a similar way. The effective viscosity rise exponentially with increasing crowder concentration for all size combinations investigated. The only difference is that the rise is stronger for larger molecules and that the scaling factor $v$ varies slightly (compare Table 4.2).

The lower effective viscosity values determined from rotational diffusion measurements of aldolase and crystallin are a first direct proof that the effective viscosity for translational and rotational diffusion can be different. Viscosity values determined from rotational diffusion measurements of aldolase are similar to those determined from translational diffusion measurements of the small dye Atto 655. The rotation of a large molecule appears to probe the crowded solution on a length scale similar to the one influencing the translation of a much smaller molecule. These results confirm the assumption that diffusion in PEG solutions is dominated by steric repulsion and hydrodynamic interactions. The behavior of crystallin is different, as viscosities determined from translational and rotational diffusion are identical for low crowder concentrations. This indicates a stronger influence of attractive interactions between $\alpha \mathrm{B}$ crystallin molecules, in agreement with previous reports ${ }^{[113]}$. In conclusion, in order to accurately predict the behavior of a molecule in a crowded environment, the interactions between a molecule and the crowder must be known.

Additionally, the results presented here provide no direct evidence for anomalous translational diffusion. Anomalous diffusion has been previously reported from FCS measurements in other crowded systems ${ }^{[10,94]}$. In the systems investigated here, diffusion seems to be normal from length scales of approximately a hundred nanometers to larger length scales. Only at length scales comparable to the size of the molecule itself, the lower viscosities determined from rotational diffusion measurements suggest that the translational diffusion might be faster than the values measured with $2 \mathrm{fFCS}$. It is likely that a simple one component system of crowders, dominated by steric repulsion, does not generate anomalous diffusion. In contrast, the cell cytoplasm is filled with long filaments and strongly interacting molecules and as such, is more likely to generate anomalous diffusion.

Future perspectives of this work include the measurements of translational and rotational diffusion of molecules in a crowded environment more closely resembling a cellular environment. For example, a mixture of PEG and stiff, long filaments such as microtubuli can be a good model system for such measurements. Moreover, it would be interesting to determine how the rotational diffusion of an elongated molecule is influenced by molecular crowding. For such shapes, molecular crowding would be expected to hinder the rotational diffusion around the long axis of the molecule much less than the rotation around its shorter axis. Taken together, the methods and results presented in this work may lead to the development of better model systems, and advance our knowledge on the physical properties of crowded systems, thereby contributing to our understanding of complex solutions such as cellular environments. 


\section{Acronyms}

\begin{tabular}{ll} 
2fFCS & Dual Focus Fluorescence Correlation Spectroscopy \\
5CB & 4-Pentyl-4'-Cyanobiphenyl \\
ACF & Autocorrelation Function \\
CCF & Cross-Correlation Function \\
CW & Continuous Wave \\
DMSO & Dimethyl Sulfoxide \\
EPR & Electron Paramagnetic Resonance \\
ER & Escape Radial \\
EtBr & Ethidium Bromide \\
FCS & Fluorescence Correlation Spectroscopy \\
FRAP & Fluorescence Recovery After Photobleaching \\
GFP & Green Fluorescent Protein \\
LC & Liquid Crystal \\
MDF & Molecular Detection Function \\
NMR & Nuclear Magnetic Resonance \\
PBS & Phosphate Buffered Saline \\
PDMS & Polydimethylsiloxane \\
PEG & Polyethylene Glycol \\
PIV & Particle Image Velocimetry \\
PRLD & Planar Radial with Line Defect \\
SPAD & Single Photon Avalanche Diode \\
SPT & Single Particle Tracking \\
\hline
\end{tabular}





\section{Bibliography}

[1] R. J. Adrian. Twenty years of particle image velocimetry. Experiments in Fluids, 39:159-169, 2005. 10.1007/s00348-005-0991-7.

[2] W.P. Ambrose, P.M. Goodwin, J. Enderlein, D.J. Semin, J.C. Martin, and R.A. Keller. Fluorescence photon antibunching from single molecules on a surface. Chemical Physics Letters, 269(3):365-370, 1997.

[3] Tadashi Ando and Jeffrey Skolnick. Crowding and hydrodynamic interactions likely dominate in vivo macromolecular motion. Proceedings of the National Academy of Sciences, 107(43):18457-18462, 2010.

[4] C. Andries and J. Clauwaert. Photon correlation spectroscopy and light scattering of eye lens proteins at high concentrations. Biophys J, 47(5):591-605, May 1985.

[5] S R Aragon and R Pecora. Fluorescence correlation spectroscopy and brownian rotational diffusion. Biopolymers, 14:119-138, 1975.

[6] Tyler J. Arbour and Jörg Enderlein. Application of dual-focus fluorescence correlation spectroscopy to microfluidic flow-velocity measurement. Lab Chip, 10(10):1286-1292, 2010.

[7] Sho Asakura and Fumio Oosawa. Interaction between particles suspended in solutions of macromolecules. Journal of Polymer Science, 33(126):183-192, 1958.

[8] Dieter Baalss and Siegfried Hess. Nonequilibrium molecular-dynamics studies on the anisotropic viscosity of perfectly aligned nematic liquid crystals. Phys. Rev. Lett., 57:86-89, Jul 1986.

[9] Frederick K. Balagaddé, Lingchong You, Carl L. Hansen, Frances H. Arnold, and Stephen R. Quake. Long-term monitoring of bacteria undergoing programmed population control in a microchemostat. Science, 309(5731):137-140, 2005.

[10] Daniel S. Banks and Cécile Fradin. Anomalous diffusion of proteins due to molecular crowding. Biophysical Journal, 89(5):2960 - 2971, 2005.

[11] Bruce J Berne and Robert Pecora. Dynamic Light Scattering. John Wiley and Sons, 1976.

[12] F.-J. Bock, H. Kneppe, and F. Schneider. Rotational viscosity of nematic liquid crystals and their shear viscosity under flow alignment. Liquid Crystals, 1(3):239-251, 1986. 
[13] J. Branebjerg, P. Gravesen, J.P. Krog, and C.R. Nielsen. Fast mixing by lamination. In Micro Electro Mechanical Systems, 1996, MEMS '96, Proceedings. 'An Investigation of Micro Structures, Sensors, Actuators, Machines and Systems'. IEEE, The Ninth Annual International Workshop on, pages 441 -446, feb 1996.

[14] David E. Budil, Keith A. Earle, and Jack H. Freed. Full determination of the rotational diffusion tensor by electron paramagnetic resonance at $250 \mathrm{ghz}$. The Journal of Physical Chemistry, 97(7):1294-1303, 1993.

[15] M Chalfie, Y Tu, G Euskirchen, WW Ward, and DC Prasher. Green fluorescent protein as a marker for gene expression. Science, 263(5148):802-805, 1994.

[16] Kelvin Chen. Microfluidics and the future of drug research. University of Toronto Journal of Undergraduate Life Sciences, 5(1):66-69, 2011.

[17] Curtis D Chin, Tassaneewan Laksanasopin, Yuk Kee Cheung, David Steinmiller, Vincent Linder, Hesam Parsa, Jennifer Wang, Hannah Moore, Robert Rouse, Gisele Umviligihozo, Etienne Karita, Lambert Mwambarangwe, Sarah L Braunstein, Janneke van de Wijgert, Ruben Sahabo, Jessica E Justman, Wafaa El-Sadr, and Samuel K Sia. Microfluidics-based diagnostics of infectious diseases in the developing world. Nat Med, 17(8):1015-1019, August 2011.

[18] Sune M. Christensen, Pierre-Yves Bolinger, Nikos S. Hatzakis, Michael W. Mortensen, and Dimitrios Stamou. Mixing subattolitre volumes in a quantitative and highly parallel manner with soft matter nanofluidics. Nat Nano, 7(1):51-55, January 2012.

[19] P. J. Collings. Liquid Crystals, Nature's Delicate Phase of Matter. Princeton University Press, 2001.

[20] Vittorio Cristini and Yung-Chieh Tan. Theory and numerical simulation of droplet dynamics in complex flows-a review. Lab Chip, 4(4):257-264, 2004.

[21] Brian R. Daniels, Richa Rikhy, Malte Renz, Terrence M. Dobrowsky, and Jennifer Lippincott-Schwartz. Multiscale diffusion in the mitotic drosophila melanogaster syncytial blastoderm. Proceedings of the National Academy of Sciences, 109(22):8588-8593, 2012.

[22] Emmanuel Dauty and A. S. Verkman. Molecular crowding reduces to a similar extent the diffusion of small solutes and macromolecules: measurement by fluorescence correlation spectroscopy. Journal of Molecular Recognition, 17(5):441-447, 2004.

[23] P. G. de Gennes and J. Prost. The Physics of Liquid Crystals. Oxford University Press, 1995.

[24] Thomas Dertinger, Victor Pacheco, Iris von der Hocht, Rudolf Hartmann, Ingo Gregor, and Jörg Enderlein. Two-focus fluorescence correlation spectroscopy: A new tool for accurate and absolute diffusion measurements. ChemPhysChem, 8(3):433-443, 2007. 
[25] K. Devanand and J. C. Selser. Asymptotic behavior and long-range interactions in aqueous solutions of poly(ethylene oxide). Macromolecules, 24(22):5943-5947, 1991.

[26] I Dierking. Textures of Liquid Crystals. Wiley, 2003.

[27] James A. Dix and A.S. Verkman. Crowding effects on diffusion in solutions and cells. Annual Review of Biophysics, 37(1):247-263, 2008. PMID: 18573081.

[28] B. Efron. Bootstrap methods: Another look at the jackknife. Annals of Statistics, 7(1):1$26,1979$.

[29] C. Eggeling, J. R. Fries, L. Brand, R. Günther, and C. A. M. Seidel. Monitoring conformational dynamics of a single molecule by selective fluorescence spectroscopy. Proceedings of the National Academy of Sciences, 95(4):1556-1561, 1998.

[30] M Ehrenberg and R Rigler. Rotational brownian-motion and fluorescence intensity fluctuations. Chemical Physics, 4:390-401, 1974.

[31] A. Einstein. Eine neue bestimmung der moleküldimensionen. Annalen der Physik, 324(2):289-306, 1906.

[32] Elliot L. Elson and Douglas Magde. Fluorescence correlation spectroscopy. i. conceptual basis and theory. Biopolymers, 13(1):1-27, 1974.

[33] Jörg Enderlein, Ingo Gregor, Digambara Patra, Thomas Dertinger, and U. Benjamin Kaupp. Performance of fluorescence correlation spectroscopy for measuring diffusion and concentration. ChemPhysChem, 6(11):2324-2336, 2005.

[34] Jörg Enderlein, Ingo Gregor, Digambara Patra, and Jörg Fitter. Art and artefacts of fluorescence correlation spectroscopy. Current Pharmaceutical Biotechnology, 5(2):155$161,2004$.

[35] Tai-Hsi Fan, Jan K. G. Dhont, and Remco Tuinier. Motion of a sphere through a polymer solution. Phys. Rev. E, 75:011803, Jan 2007.

[36] Richard Feynman. Feynman's lectures on physics. Addison-Wesley, 1964.

[37] Wikimedia Foundation. (wikimedia commons). http://commons.wikimedia.org.

[38] K Fushimi and A S Verkman. Low viscosity in the aqueous domain of cell cytoplasm measured by picosecond polarization microfluorimetry. The Journal of Cell Biology, 112(4):719-725, 1991.

[39] Ana I. García-Pérez, Emilio A. López-Beltrán, Peter Klüner, Jose Luque, Paloma Ballesteros, and Sebastián Cerdán. Molecular crowding and viscosity as determinants of translational diffusion of metabolites in subcellular organelles. Archives of Biochemistry and Biophysics, 362(2):329-338, February 1999. 
[40] Chris Gell, David J. Brockwell, Godfrey S. Beddard, Sheena E. Radford, Arnout P. Kalverda, and D. Alastair Smith. Accurate use of single molecule fluorescence correlation spectroscopy to determine molecular diffusion times. Single Molecules, 2(3):177$181,2001$.

[41] David S. Goodsell. Escherichia coli. Biochemistry and Molecular Biology Education, 37(6):325-332, 2009.

[42] P Gravesen, J Branebjerg, and O S Jensen. Microfluidics-a review. Journal of Micromechanics and Microengineering, 3(4):168, 1993.

[43] Michael Gösch, Hans Blom, Johan Holm, Toni Heino, and Rudolf Rigler. Hydrodynamic flow profiling in microchannel structures by single molecule fluorescence correlation spectroscopy. Analytical Chemistry, 72(14):3260-3265, 2000. PMID: 10939397.

[44] J. B. Gurdon, A. Mitchell, and D. Mahony. Direct and continuous assessment by cells of their position in amorphogen gradient. Nature, 376(6540):520-521, August 1995.

[45] Taekjip Ha, Ted A. Laurence, Daniel S. Chemla, and Shimon Weiss. Polarization spectroscopy of single fluorescent molecules. The Journal of Physical Chemistry B, 103(33):6839-6850, 1999.

[46] Benjamin D. Hamlington, Benjamin Steinhaus, James J. Feng, Darren Link, Michael J. Shelley, and Amy Q. Shen. Liquid crystal droplet production in a microfluidic device. Liquid Crystals, 34(7):861-870, 2007.

[47] Ulrich Haupts, Sudipta Maiti, Petra Schwille, and Watt W. Webb. Dynamics of fluorescence fluctuations in green fluorescent protein observed by fluorescence correlation spectroscopy. Proceedings of the National Academy of Sciences, 95(23):13573-13578, 1998.

[48] Mei He and Amy E Herr. Automated microfluidic protein immunoblotting. Nat. Protocols, 5(11):1844-1856, October 2010.

[49] D.B. Holmes and J.R. Vermeulen. Velocity profiles in ducts with rectangular cross sections. Chemical Engineering Science, 23(7):717 - 722, 1968.

[50] Robert Holyst, Anna Bielejewska, Jedrzej Szymanski, Agnieszka Wilk, Adam Patkowski, Jacek Gapinski, Andrzej Zywocinski, Tomasz Kalwarczyk, Ewelina Kalwarczyk, Marcin Tabaka, Natalia Ziebacz, and Stefan A. Wieczorek. Scaling form of viscosity at all length-scales in poly(ethylene glycol) solutions studied by fluorescence correlation spectroscopy and capillary electrophoresis. Phys. Chem. Chem. Phys., 11(40):9025-9032, 2009.

[51] J Horwitz. Alpha-crystallin can function as a molecular chaperone. Proceedings of the National Academy of Sciences, 89(21):10449-10453, 1992.

[52] Joseph Horwitz. Alpha-crystallin. Experimental Eye Research, 76(2):145 - 153, 2003. 
[53] Songwan Jin and A. S. Verkman. Single particle tracking of complex diffusion in membranes: Simulation and detection of barrier, raft, and interaction phenomena. The Journal of Physical Chemistry B, 111(14):3625-3632, 2007. PMID: 17388520.

[54] H P Kao, J R Abney, and A S Verkman. Determinants of the translational mobility of a small solute in cell cytoplasm. The Journal of Cell Biology, 120(1):175-184, 1993.

[55] Haruo Kasai and Ole H. Petersen. Spatial dynamics of second messengers: Ip3 and camp as long-range and associative messengers. Trends in Neurosciences, 17(3):95$101,1994$.

[56] P Kask, P Piksarv, U Mets, M Pooga, and E Lippmaa. Fluorescence correlation spectroscopy in the nanosecond time range: rotational diffusion of bovine carbonic anhydrase b. Eur Biophys J, 14:257-261, 1987.

[57] P Kask, P Piksarv, M Pooga, U Mets, and E Lippmaa. Separation of the rotational contribution in fluorescence correlation experiments. Biophys J, 55(2):213-220, Feb 1989.

[58] Ulrich Kettling, Andre Koltermann, Petra Schwille, and Manfred Eigen. Real-time enzyme kinetics monitored by dual-color fluorescence cross-correlation spectroscopy. Proceedings of the National Academy of Sciences, 95(4):1416-1420, 1998.

[59] Duncan Kilburn, Joon Ho Roh, Liang Guo, Robert M. Briber, and Sarah A. Woodson. Molecular crowding stabilizes folded rna structure by the excluded volume effect. Journal of the American Chemical Society, 132(25):8690-8696, 2010. PMID: 20521820.

[60] H. Kneppe, F. Schneider, and N. K. Sharma. A comparative study of the viscosity coefficients of some nematic liquid crystalss. Berichte der Bunsengesellschaft für physikalische Chemie, 85(8):784-789, 1981.

[61] James B. Knight, Ashvin Vishwanath, James P. Brody, and Robert H. Austin. Hydrodynamic focusing on a silicon chip: Mixing nanoliters in microseconds. Phys. Rev. Lett., 80:3863-3866, Apr 1998.

[62] S.H. Koenig, R.D. Brown, M. Spiller, B. Chakrabarti, and A. Pande. Intermolecular protein interactions in solutions of calf lens alpha-crystallin. results from $1 / \mathrm{t} 1$ nuclear magnetic relaxation dispersion profiles. Biophys J, 61(3):776-785, March 1992.

[63] Jonas Korlach, Petra Schwille, Watt W. Webb, and Gerald W. Feigenson. Characterization of lipid bilayer phases by confocal microscopy and fluorescence correlation spectroscopy. Proceedings of the National Academy of Sciences, 96(15):8461-8466, 1999.

[64] S. Kralj and S. Zumer. The saddle-splay surface elasticity of nematic structures confined to a cylindrical capillary. Phys. Rev. E., 51:366-379, 1995. 
[65] Christopher D. Kroenke, J. Patrick Loria, Larry K. Lee, Mark Rance, and Arthur G. Palmer. Longitudinal and transverse ${ }^{1} \mathrm{~h}-{ }^{15} \mathrm{n}$ dipolar $/{ }^{15} \mathrm{n}$ chemical shift anisotropy relaxation interference: Unambiguous determination of rotational diffusion tensors and chemical exchange effects in biological macromolecules. Journal of the American Chemical Society, 120(31):7905-7915, 1998.

[66] Kalyan K. Kuricheti, Volker Buschmann, and Kenneth D. Weston. Application of fluorescence correlation spectroscopy for velocity imaging in microfluidic devices. Appl. Spectrosc., 58(10):1180-1186, Oct 2004.

[67] Joseph R. Lakowicz. Principles of Fluorescence Spectroscopy. Springer, 2006.

[68] Arthur D Lander, Qing Nie, and Frederic Y.M Wan. Do morphogen gradients arise by diffusion? Dev Cell, 2(6):785-796, June 2002.

[69] W. R. Lempert, P. Ronney, K. Magee, K. R. Gee, and R. P. Haugland. Flow tagging velocimetry in incompressible flow using photo-activated nonintrusive tracking of molecular motion (phantomm). Experiments in Fluids, 18:249-257, 1995. 10.1007/BF00195095.

[70] F. M. Leslie. Some constitutive equations for liquid crystals. Archive for Rational Mechanics and Analysis, 28:265-283, 1968. 10.1007/BF00251810.

[71] Anastasia Loman, Ingo Gregor, Christina Stutz, Markus Mund, and Jörg Enderlein. Measuring rotational diffusion of macromolecules by fluorescence correlation spectroscopy. Photochem. Photobiol. Sci., 9(5):627-636, 2010.

[72] J. R. Lombardi, J. W. Raymonda, and A. C. Albrecht. Rotational relaxation in rigid media by polarized photoselection. The Journal of Chemical Physics, 40(4):1148-1156, 1964.

[73] Gino De Luca and Alejandro D. Rey. Point and ring defects in nematics under capillary confinement. The Journal of Chemical Physics, 127(10):104902, 2007.

[74] Douglas Magde, Elliot Elson, and W. W. Webb. Thermodynamic fluctuations in a reacting system - measurement by fluorescence correlation spectroscopy. Phys. Rev. Lett., 29:705-708, Sep 1972.

[75] Douglas Magde, Elliot L. Elson, and Watt W. Webb. Fluorescence correlation spectroscopy. ii. an experimental realization. Biopolymers, 13(1):29-61, 1974.

[76] Douglas Magde, Watt W. Webb, and Elliot L. Elson. Fluorescence correlation spectroscopy. iii. uniform translation and laminar flow. Biopolymers, 17(2):361-376, 1978.

[77] Andi Mainz, Stefan Jehle, Barth J. van Rossum, Hartmut Oschkinat, and Bernd Reif. Large protein complexes with extreme rotational correlation times investigated in solution by magic-angle-spinning nmr spectroscopy. Journal of the American Chemical Society, 131(44):15968-15969, 2009. PMID: 19839609. 
[78] Anne Martel, Manfred Burghammer, Richard Davies, Emanuela DiCola, Pierre Panine, Jean-Baptiste Salmon, and Christian Riekel. A microfluidic cell for studying the formation of regenerated silk by synchrotron radiation small- and wide-angle x-ray scattering. Biomicrofluidics, 2(2):024104, 2008.

[79] C. D. Meinhart, S. T. Wereley, and J. G. Santiago. Piv measurements of a microchannel flow. Experiments in Fluids, 27:414-419, 1999. 10.1007/s003480050366.

[80] K B Merck, P J Groenen, C E Voorter, W A de Haard-Hoekman, J Horwitz, H Bloemendal, and W W de Jong. Structural and functional similarities of bovine alpha-crystallin and mouse small heat-shock protein. a family of chaperones. Journal of Biological Chemistry, 268(2):1046-1052, 1993.

[81] C. Monat, P. Domachuk, and B. J. Eggleton. Integrated optofluidics: A new river of light. Nat Photon, 1(2):106-114, February 2007.

[82] Saravanakumar Narayanan, Bram Kamps, Wilbert C. Boelens, and Bernd Reif. $\alpha$ bcrystallin competes with alzheimer's disease $\beta$-amyloid peptide for peptide-peptide interactions and induces oxidation of abeta-met35. FEBS Letters, 580(25):5941 - 5946, 2006.

[83] J. A. Nelder and R. Mead. A simplex method for function minimization. The Computer Journal, 7(4):308-313, 1965.

[84] John Olmsted and David R. Kearns. Mechanism of ethidium bromide fluorescence enhancement on binding to nucleic acids. Biochemistry, 16(16):3647-3654, 1977.

[85] Rami A. Omari, Andrew M. Aneese, Christopher A. Grabowski, and Ashis Mukhopadhyay. Diffusion of nanoparticles in semidilute and entangled polymer solutions. The Journal of Physical Chemistry B, 113(25):8449-8452, 2009. PMID: 19476342.

[86] Christoph M Pieper and Jörg Enderlein. Fluorescence correlation spectroscopy as a tool for measuring the rotational diffusion of macromolecules. Chemical Physics Letters, 516(1-3):1-11, 2011.

[87] Demetri Psaltis, Stephen R. Quake, and Changhuei Yang. Developing optofluidic technology through the fusion of microfluidics and optics. Nature, 442(7101):381-386, July 2006.

[88] E M Purcell. Life at low reynolds number. Am. J. Phys., 45(1):3-11, 1977.

[89] Sergey P. Radko and Andreas Chrambach. Electrophoresis of proteins in semidilute polyethylene glycol solutions: Mechanism of retardation. Biopolymers, 42(2):183-189, 1997.

[90] Barbara Rauer, Eberhard Neumann, Jerker Widengren, and Rudolf Rigler. Fluorescence correlation spectrometry of the interaction kinetics of tetramethylrhodamin $\alpha$ bungarotoxin with torpedo californica acetylcholine receptor. Biophysical Chemistry, 58(1-2):3-12, 1996. 
[91] R Rigler and J Widengren. Ultrasensitive detection of single molecules by fluorescence correlation spectroscopy. Bioscience, 3:180-183, 1990.

[92] GermÃ $i n$ Rivas, Javier A. Fernandez, and Allen P. Minton. Direct observation of the self-association of dilute proteins in the presence of inert macromolecules at high concentration via tracer sedimentation equilibrium: Theory, experiment, and biological significance. Biochemistry, 38(29):9379-9388, 1999.

[93] S. Rüttinger, V. Buschmann, V., B. Krämer, R. Erdmann, R. Macdonald, and F. Koberling. Comparison and accuracy of methods to determine the confocal volume for quantitative fluorescence correlation spectroscopy. Journal of Microscopy, 232(2):343-352, 2008.

[94] Hugo Sanabria, Yoshihisa Kubota, and M. Neal Waxham. Multiple diffusion mechanisms due to nanostructuring in crowded environments. Biophysical Journal, 92(1):313 $-322,2007$.

[95] J. G. Santiago, S. T. Wereley, C. D. Meinhart, D. J. Beebe, and R. J. Adrian. A particle image velocimetry system for microfluidics. Experiments in Fluids, 25:316-319, 1998. $10.1007 / \mathrm{s} 003480050235$.

[96] Ralf Seemann, Martin Brinkmann, Thomas Pfohl, and Stephan Herminghaus. Droplet based microfluidics. Reports on Progress in Physics, 75(1):016601, 2012.

[97] Shahab Shojaei-Zadeh and Shelley L. Anna. Role of surface anchoring and geometric confinement on focal conic textures in smectic-a liquid crystals. Langmuir, 22(24):9986-9993, 2006. PMID: 17106990.

[98] G.J.J. Stege, K. Renkawek, P.S.G. Overkamp, P. Verschuure, A.F. van Rijk, A. ReijnenAalbers, W.C. Boelens, G.J.C.G.M. Bosman, and W.W. de Jong. The molecular chaperone $\alpha$ b-crystallin enhances amyloid $\beta$ neurotoxicity. Biochemical and Biophysical Research Communications, 262(1):152 - 156, 1999.

[99] Anish Tuteja, Michael E. Mackay, Suresh Narayanan, Subashini Asokan, and Michael S. Wong. Breakdown of the continuum stokes-einstein relation for nanoparticle diffusion. Nano Letters, 7(5):1276-1281, 2007. PMID: 17397233.

[100] Alan S Verkman. Solute and macromolecule diffusion in cellular aqueous compartments. Trends in Biochemical Sciences, 27(1):27 - 33, 2002.

[101] A. Vrij. Polymers at interfaces and the interactions in colloidal dispersions. Pure Appl. Chem., 48(4):471-483, 1976.

[102] Michael Wahl, Ingo Gregor, Mattias Patting, and Jörg Enderlein. Fast calculation of fluorescence correlation data with asynchronous time-correlated single-photon counting. Opt. Express, 11(26):3583-3591, Dec 2003. 
[103] P Wahl, J Paoletti, and J B Le Pecq. Decay of fluorescence emission anisotropy of the ethidium bromide-dna complex. evidence for an internal motion in dna. Proc Natl Acad Sci U S A, 65(2):417-421, Feb 1970.

[104] G. R. Wang. Laser induced fluorescence photobleaching anemometer for microfluidic devices. Lab Chip, 5(4):450-456, 2005.

[105] Thomas Ward, Magalie Faivre, Manouk Abkarian, and Howard A. Stone. Microfluidic flow focusing: Drop size and scaling in pressure versus flow-rate-driven pumping. ELECTROPHORESIS, 26(19):3716-3724, 2005.

[106] G Weber. Polarization of the fluorescence of macromolecules. ii. fluorescent conjugates of ovalbumin and bovine serum albumin. Biochem J, 51(2):155-167, May 1952.

[107] G Weber. Rotational brownian motion and polarization of the fluorescence of solutions. Adv. Protein Chem., 8:415-459, 1953.

[108] Shimon Weiss. Measuring conformational dynamics of biomolecules by single molecule fluorescence spectroscopy. Nat. Struct. Mol. Biol., 7(9):724-729, September 2000.

[109] George M. Whitesides. The origins and the future of microfluidics. Nature, 442(7101):368-373, July 2006.

[110] Jerker Widengren, Ülo Mets, and Rudolf Rigler. Photodynamic properties of green fluorescent proteins investigated by fluorescence correlation spectroscopy. Chemical Physics, 250(2):171 - 186, 1999.

[111] Jerker Widengren, Uelo Mets, and Rudolf Rigler. Fluorescence correlation spectroscopy of triplet states in solution: a theoretical and experimental study. The Journal of Physical Chemistry, 99(36):13368-13379, 1995.

[112] Jerker Widengren and Petra Schwille. Characterization of photoinduced isomerization and back-isomerization of the cyanine dye cy 5 by fluorescence correlation spectroscopy. The Journal of Physical Chemistry A, 104(27):6416-6428, 2000.

[113] J.Z. Xia, T. Aerts, K. Donceel, and J. Clauwaert. Light scattering by bovine alphacrystallin proteins in solution: hydrodynamic structure and interparticle interaction. Biophys J, 66(3):861-872, March 1994.

[114] Qiaobing Xu, Michinao Hashimoto, Tram T. Dang, Todd Hoare, Daniel S. Kohane, George M. Whitesides, Robert Langer, and Daniel G. Anderson. Preparation of monodisperse biodegradable polymer microparticles using a microfluidic flow-focusing device for controlled drug delivery. Small, 5(13):1575-1581, 2009.

[115] J Yguerabide, H F Epstein, and L Stryer. Segmental flexibility in an antibody molecule. J Mol Biol, 51(3):573-590, Aug 1970. 



\section{Acknowledgments}

First of all, I would like to thank my supervisor, Jörg Enderlein, for the opportunity to undertake the journey that has lead to this thesis. He has not only been a great mentor but also a good friend in this time. Besides ample time to discuss scientific problems and new ideas, he often found the time for culinary explorations and recreational activities.

I greatly appreciate the advice and guidance of my thesis committee members, Professor Helmut Grubmüller and Professor Tim Salditt. I would like to thank them both very much, not only for taking the time to regularly monitor and discuss the progress of this work, but also for their genuine interest and encouragement.

I would like to thank my traveling companions Mira, Kerstin, Anja, Stefi, Phillip, Qui, Ingo, Bernd, Olaf, Manoj, Alexey and Dirk for the great working environment and fun atmosphere in the lab. I wish you all the best for your future travels, may they be long and prosperous. Special thanks go to Kerstin, Olaf, Qui, and Ingo for their suggestions and helpful discussions during the creation of this thesis. Additionally, the secretaries and workshop staff of the Third Physical Institute have been extremely helpful in organizing matters and the construction of material needed for this work.

Many thanks also go to the whole GGNB team. Studying in Göttingen and being part of GGNB has been a stimulating and fun experience.

I am grateful to my family and especially my parents for supporting my choices and encouraging me. Not only have they made this journey possible, but their love and advice has helped me through any difficulties along the way. Most of all I have to thank Konstantina for always being there for me, her love and support during these last years.

Furthermore, I thank Andi Mainz from the Leibniz-Institut für Molekulare Pharmakologie (FMP) in Berlin for sharing his alphaB-crystallin samples so generously with me and Anupam Sengupta from the Max-Planck-Institute for Dynamics and Self-Organization in Göttingen for the microfluidic devices and liquid crystal samples.

I acknowledge the financial support of this work by a GGNB bridging fund (GGNB) and the SFB 860 (DFG). 
